to appear in Annual Reviews of Astronomy and Astrophysics, Vol. 45, 2007

\title{
Toward Understanding Massive Star Formation
}

\author{
Hans Zinnecker ${ }^{1} \quad$ Harold W. Yorke 2
}

\begin{abstract}
Although fundamental for astrophysics, the processes that produce massive stars are not well understood. Large distances, high extinction, and short timescales of critical evolutionary phases make observations of these processes challenging. Lacking good observational guidance, theoretical models have remained controversial. This review offers a basic description of the collapse of a massive molecular core and a critical discussion of the three competing concepts of massive star formation:

- monolithic collapse in isolated cores

- competitive accretion in a protocluster environment

- stellar collisions and mergers in very dense systems

We also review the observed outflows, multiplicity, and clustering properties of massive stars, the upper initial mass function and the upper mass limit. We conclude that high-mass star formation is not merely a scaledup version of low-mass star formation with higher accretion rates, but partly a mechanism of its own, primarily owing to the role of stellar mass and radiation pressure in controlling the dynamics.
\end{abstract}

Key Words accretion, circumstellar disks, HII regions, massive stars, protostars, star formation

\footnotetext{
${ }^{1}$ Astrophysikalisches Institut Potsdam, An der Sternwarte 16, D-14482 Potsdam, Germany; email: hzinnecker@aip.de

2 Jet Propulsion Laboratory, California Institute of Technology, 4800 Oak Grove Drive, Pasadena, CA 91109 USA; email: Harold.Yorke@jpl.nasa.gov
} 


\section{Contents}

1 INTRODUCTION

1.1 Basic Issues . . . . . . . . . . . . . . . . . 5

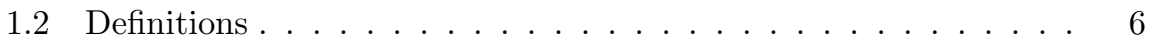

1.3 Recommended Reading . . . . . . . . . . . . . . 8

1.4 The Focus of This Review . . . . . . . . . . . . . . . . . . . . . . 9 9

\section{MASSIVE STAR FORMATION:}

KEY OBSERVATIONS 11

2.1 Observable Stages . . . . . . . . . . . . . . . . 11

2.2 Initial Conditions . . . . . . . . . . . . . . . . . . . 12

2.3 Endproducts ...................... 13

2.3.1 OB Clusters .................... 13

2.3.2 OB Associations ................ 14

2.3.3 Field OB Stars . . . . . . . . . . . . . . 14

2.4 Clues from multiplicity . . . . . . . . . . . . . . 15

2.5 Upper Initial Mass Function and Upper Mass Limit . . . . . . . 18

2.6 Feedback and Triggering . . . . . . . . . . . . 21

\section{MASSIVE STAR FORMATION:}

BASIC THEORY 22

3.1 Sequence of Events . . . . . . . . . . . . . . . 22

3.2 The Compression Phase . . . . . . . . . . . . . . 23

3.3 The Collapse Phase . . . . . . . . . . . . . . . . 27

3.4 The Accretion Phase . . . . . . . . . . . . . 27

3.4.1 Formation of the Hydrostatic Core . . . . . . . . . . . . 27

3.4.2 Evolution of Accreting Cores . . . . . . . . . . . 28

3.5 Overcoming Radiative Acceleration . . . . . . . . . . 31

3.5.1 Reduce $\kappa_{\mathrm{eff}}$...................... 32

3.5.2 Reduce the Effective Luminosity . . . . . . . . . . . . 34

3.5.3 Increase Gravity . . . . . . . . . . . . . . . 36

3.6 Stellar and Protostellar Luminosity Evolution . . . . . . . . . . . 36

3.7 Stellar Evolution Beyond the Zero Age Main Sequence . . . . . . 38 
3.8 Ionization Evolution and Cloud Disruption $\ldots \ldots \ldots \ldots$

4 MASSIVE STAR FORMATION: COMPETING CONCEPTS AND CALCULATIONS 43

4.1 Monolithic Collapse and Disk Accretion . . . . . . . . . . 43

4.2 Competitive Accretion and Runaway Growth . . . . . . . . 45

4.3 Stellar Collisions and Mergers . . . . . . . . . . . . . . 48

4.4 Triggered OB Star Formation . . . . . . . . . . . . . 50

4.5 Dynamical Evolution: Mass Segregation and Runaway OB Stars 54 4.5.1 Mass Segregation . . . . . . . . . . . . 54

4.5.2 Runaway OB Stars . . . . . . . . . . . . . . . 54

5 MASSIVE STAR FORMATION:

BINARY AND MULTIPLE SYSTEMS

5.1 Disk or Filament Fragmentation . . . . . . . . . . . . 56

5.2 Accretion onto a Low-Mass, Wide Binary . . . . . . . . . . 57

5.3 Failed Mergers in Stellar Collisions . . . . . . . . . . 58

5.4 Disk-Assisted Capture . . . . . . . . . . . . . . . . . . 59

5.5 N-Body Dynamical Evolution . . . . . . . . . . . . . . . 60

5.6 The Origin of Trapezium Systems . . . . . . . . . . . . . 61

6 MASSIVE STAR FORMATION: DISCUSSION 62

6.1 Disks and Outflows . . . . . . . . . . . . . . . 62

6.2 Binary Statistics and the Most Massive Binary Systems . . . . 65

6.3 The Universality of the Upper Initial Mass Function . . . . . . 69

6.4 The Number of Accreting Massive Protostars in the Galaxy . . . 71

6.5 Is There a Maximum Stellar Mass Set by Star Formation? . . . . 73

6.6 Evolutionary Sequence . . . . . . . . . . . . . . . . . . 755

7 WHY HIGH-MASS STAR FORMATION IS NOT A SCALEDUP VERSION OF LOW-MASS STAR FORMATION

8 OUTLOOK: RELEVANT FUTURE OBSERVATIONS 80

9 FINAL SUMMARY 83 
10 APPENDIX - NEARBY ( $<1 \mathrm{kpc})$

MASSIVE STAR-FORMATION REGIONS

11 GLOSSARY 


\section{INTRODUCTION}

\subsection{Basic Issues}

Massive stars play a key role in the evolution of the Universe. They are the principal source of heavy elements and UV radiation. Through a combination of winds, massive outflows, expanding HII regions, and supernova explosions they provide an important source of mixing and turbulence in the interstellar medium (ISM) of galaxies. Turbulence in combination with differential rotation drives galactic dynamos. Galactic magnetic fields are generated, interacting with supernova shock fronts that accelerate cosmic rays. Cosmic rays, UV radiation, and dissipation of turbulence are the principal sources of heating in the ISM, whereas heavy elements found in dust, molecules, and in atomic/ionic form ultimately are responsible for its cooling. Massive stars thus profoundly affect the star- and planet-formation process (Bally, Moeckel \& Throop 2005) as well as the physical, chemical, and morphological structure of galaxies (e.g., Kennicutt 1998, 2005).

In spite of the dominant role that massive stars play in shaping galactic structure and evolution, our understanding of their formation and early evolution is still sketchy. There are many reasons. High dust extinction makes it difficult to observe high-mass stars during critical early formation phases. They are rare. They evolve quickly and important evolutionary phases are shortlived. The theoretical problem is extremely complex. Finally, massive stars are seldomly (if at all) formed in isolation; the proximity of other high-mass stars compounds the complex influence of the forming star on its local environment via gravitational interactions, powerful outflows and winds, ionizing radiation, and supernovae.

The low number statistics of young or forming high-mass stars is only partially offset by their higher luminosities, which allow us to study them at greater distances than their low-mass counterparts. However, insufficient spatial resolution is an issue - an entire OB-star cluster is often contained in a single observing pixel (e.g., Henning \& Stecklum 2002). 


\subsection{Definitions}

Star formation typically starts with a collapsing gas condensation (core) inside a larger subunit (clump) of a molecular cloud (cf. Williams, Blitz \& McKee 2000). A protostar forms that increases its mass by accretion (accumulation) of neighboring gas, while at the same time some mass loss occurs through a bipolar outflow and/or a collimated jet. Let us define some of the terminology adopted here.

One of the most misused terms in papers dealing with star formation is protostar, which is considered the Holy Grail (Wynn-Williams 1982) of IR astronomy. Here, we reserve the term protostar or protostellar object for a gaseous object in hydrostatic equilibrium (gas pressure forces balance the gas' self-gravity), which has not yet begun hydrogen burning but which will, given time, burn hydrogen. At the point hydrogen burning commences, we shall speak of a zero-age main-sequence (ZAMS) star; as long as hydrogen burning occurs in the center, we shall speak of a main-sequence star. Note that the size scale of a protostar is at most a few tens of solar radii.

We use the terms massive star and high-mass star interchangeably to denote an OB star sufficiently massive to produce a type II supernova $\left(\mathrm{M}_{*} / \mathrm{M}_{\odot} \gtrsim 8\right.$ for solar abundances). With these definitions in mind, the term high-mass protostar denotes a $\gtrsim 8 \mathrm{M}_{\odot}$ hydrostatic object that has not yet begun hydrogen burning. As we shall see in the following, such objects exist only briefly during a transitory stage between "accreting intermediate-mass protostar" and "accreting high-mass star." Because it will be impossible to distinguish observationally when an accreting object begins burning hydrogen, we suggest that the terms massive protostar and high-mass protostar generally be avoided.

In Table 1, we give a crude classification of massive stars in terms of logarithmic mass intervals and the corresponding main sequence spectral types.

We reserve the terms very massive star (VMS) and supermassive star (SMS) for stars in the mass ranges of $100 \lesssim \mathrm{M}_{*} / \mathrm{M}_{\odot} \lesssim 1000$ and $10^{4} \lesssim \mathrm{M}_{*} / \mathrm{M}_{\odot} \lesssim 10^{8}$, respectively, and introduce the term ultramassive star (UMS) for stars in the mass range of $10^{3} \lesssim \mathrm{M}_{*} / \mathrm{M}_{\odot} \lesssim 10^{4}$. SMSs are equilibrium configurations that are dominated by radiation pressure - baryons and electron-positron pairs provide 
Table 1: Main Sequence massive star definition (logarithmic mass ranges)

\begin{tabular}{ccc}
\hline \hline Mass & Designation & Sp. type \\
\hline $8-16 \mathrm{M}_{\odot}$ & Early B-type massive stars & B3V to B0V \\
$16-32 \mathrm{M}_{\odot}$ & Late O-type massive stars & O9V to O6V \\
$32-64 \mathrm{M}_{\odot}$ & Early O-type massive stars & $05 \mathrm{~V}$ to O2V \\
$64-128 \mathrm{M}_{\odot}$ & O/WR-type massive stars & ${\mathrm{WNL}-\mathrm{H}^{b}}$ \\
\hline
\end{tabular}

${ }^{a} \mathrm{O} 2 \mathrm{~V}$ main sequence stars have been identified by Walborn et al. (2002).

${ }^{b}$ WNL-H: N-rich late-type Wolf-Rayet (WR) stars, still on the

Main Sequence (H-burning) - see Crowther (2007).

only a minor contribution to the equation of state. At some point during their evolution SMSs collapse owing to a general relativistic gravitational instability. Whereas in the present epoch VMSs, UMSs, and SMSs are unlikely to be formed except under very special conditions, stars with masses in excess of $100 \mathrm{M}_{\odot}$ are expected during the first epoch of star formation (Bromm \& Larson 2004; Abel, Bryan \& Norman 2000). VMSs, UMSs, and SMSs are not discussed in this review. A recent discussion of the formation and evolution of VMSs is given in Portegies Zwart et al. (2006) and in Belkus, van Bever \& Vanbeveren (2007).

What is accretion? The term accretion is used in a variety of senses. Measured accretion rates often refer to the rate of mass inflow toward star-forming sites - not the rate at which a star or protostar gains mass. Originating from a $0.1 \mathrm{pc}$ scale, this material cannot possibly fall into a sub- $10^{-6}$ pc region without carrying significant angular momentum. Instead, it either forms a disk or hits and is mixed with prior existing disk material (see Figure 1). Thus, we distinguish between the accretion of cloud core material onto a disk $\left(\dot{\mathrm{M}}_{\mathrm{D}-\mathrm{acc}}\right)$ and the accretion onto a (proto-) $\operatorname{star}\left(\dot{\mathrm{M}}_{\mathrm{S}-\mathrm{acc}}\right)$.

Analogous to accretion, mass loss is used in a variety of senses. Measured mass loss rates from jets and outflows do not necessarily reflect the mass loss from an isolated young star. We thus distinguish between the mass loss from the (proto-)star via a wind $\left(\dot{\mathrm{M}}_{\mathrm{S}-\text { wind }}\right)$, the mass loss launched from the accretion disk $\left(\dot{\mathrm{M}}_{\mathrm{D}-\text { wind }}\right)$, which never reaches the (proto-)star, and the material swept up 
into the outflow from the surrounding molecular cloud $\left(\dot{\mathrm{M}}_{\text {load }}\right)$. The measured outflow could have contributions from several stars and several disks.

The interrelation between disk accretion and disk winds is a fascinating aspect of massive star formation (see, e.g., the recent magneto-hydrodynamic models of Banerjee \& Pudritz 2007) - and is at the focus of the frequently asked question: Is high-mass star formation a scaled-up version of low-mass star formation? The answer to this question will be better defined by the time we reach the end of this review.

\subsection{Recommended Reading}

The study of the origin of massive stars is a relatively new field of astrophysical research. There is no comprehensive monograph on the subject, but there are several conference proceedings over the past few years dedicated to the topic, of which we recommend the following:

- Massive Stars: Their Lives in the interstellar Medium (Cassinelli \& Churchwell 1993)

- Hot Star Workshop III: The Earliest Stages of Massive Star Birth (Crowther 2002)

- Massive Star Birth: A Crossroads of Astrophysics (Cesaroni et al. 2005a)

The reviews on Environment and Formation of Massive Stars (Garay \& Lizano 1999), Control of Star Formation by Supersonic Turbulence (Mac Low \& Klessen 2004), The Formation of the First Stars in the Universe (Glover 2005), The Birth of Massive Stars and Star Clusters (Tan 2005), High Mass Star Formation by Gravitational Collapse of Massive Cores (Krumholz 2007), and The Critical Role of Disks in the Formation of High-Mass Stars (Cesaroni et al. 2007) are also recommended. Among the reviews in the Proceedings of Protostars and Planets V, we particularly recommend The Formation of Massive Stars (Beuther et al. 2007).

There are also a few related Annual Reviews articles:

- Compact HII Regions and OB Star Formation (Habing \& Israel 1979) 
- The Search for Infrared Protostars (Wynn-Williams 1982)

- The Dynamic Evolution of HII Regions - Recent Theoretical Developments (Yorke 1986)

- The Orion Molecular Cloud and Star-Forming Region (Genzel \& Stutzki 1989)

- Physical Conditions in Regions of Star Formation (Evans 1999)

- Ultra-Compact HII Regions and Massive Star Formation (Churchwell 2002)

- Massive Stars in the Local Group: Implications for Stellar Evolution and Star Formation (Massey 2003)

- The First Stars (Bromm \& Larson 2004)

Except for the last, none of these earlier reviews had its focus on the formation aspect of massive stars but rather provided a descriptive observational summary of the properties of young OB stars and their HII regions. The present Annual Reviews article will be accompanied in the same volume by an article on 'Physical properties of Wolf-Rayet stars' (Crowther 2007) and an article on 'Theory of Star Formation' (McKee \& Ostriker 2007) which mostly addresses low-mass star formation, but also includes an important section on high-mass star formation.

\subsection{The Focus of This Review}

The major questions we wish to address in this review are:

1. What is the sequence of observable states leading from molecular clouds to young high-mass stars?

2. What are the initial conditions of massive star formation (gas densities, temperatures, clump masses, etc.) and how do they come about?

3. Do massive stars always form in dense stellar clusters or can they form in isolation? What special conditions are necessary to allow coalescence, i.e., mergers of stars? 
4. Which clues to the origin can be gleaned from multiplicity observations? How do we explain the very tight massive spectroscopic binaries and OB runaway stars?

5. How does the forming massive star influence its immediate surroundings, possibly limiting its final mass and/or the final mass of its neighbors?

6. How do young massive stars influence their global environment, either by inhibiting or by triggering further star formation? How do we get a starburst?

To tackle these questions, we first discuss some key observations related to massive star formation. 


\section{MASSIVE STAR FORMATION: KEY OBSERVATIONS}

\subsection{Observable Stages}

The optically visible main-sequence life of OB-type stars is preceded by an embedded phase that lasts about $15 \%$ of their lifetime (Churchwell 2002). As summarized by Menten, Pillai \& Wyrowski (2005) and van der Tak \& Menten (2005), observations at mid-IR through radio wavelengths have shown that this embedded phase can be subdivided into several groups of objects:

- IR dark clouds (Perault et al. 1996, ISOCAM; Egan et al. 1998, MSX; Benjamin et al. 2003, Spitzer). Their internal density maxima and temperature minima likely represent the initial conditions of high-mass star formation; a compilation of several dozens of such high-mass starless cores has been given by Sridharan, Williams \& Fuller (2005). Some of these cores probably contain low-mass and intermediate-mass accreting protostars, which are faint and hard to detect. Protostellar outflow activity has been detected in one of them (Beuther, Sridharan \& Saito 2005).

- Hot molecular cores (Kurtz et al. 2000, Cesaroni 2005). These have large masses of warm and dense gas, and large abundances of complex organic molecules evaporated off dust grains; they are signposted by methanol maser emission (Menten 1991, Walsh et al. 1998, Hill et al. 2005); ground-based detectability on the Wien part of the spectral energy distribution with sufficient spatial resolution is difficult (Stecklum et al. 2002), but comes into reach with dedicated 8-m class telescope observations (De Buizer \& Minier 2005, Linz et al. 2005).

- Hypercompact and ultracompact HII regions (Kurtz 2005, Hoare et al. 2007). In these regions, small but growing pockets of ionized gas have developed that stay confined to the stellar vicinity. Whereas hypercompact HII regions probably represent individual photoevaporating disks (Keto 2007; see also the example in Nielbock et al. 2007), ultracompact HII 
regions probably represent disk-less stars photoionizing their own cocoons and massive envelopes.

- Compact and classical HII regions (Mezger et al. 1967, Yorke 1986). Their gas is ionized globally, often by several ionizing sources. It expands hydrodynamically as a whole and disrupts the parent molecular cloud, revealing both the embedded high-mass and lower mass stellar population for optical and near-IR observations (Carpenter et al. 1993; Zinnecker, McCaughrean \& Wilking 1993).

\section{$2.2 \quad$ Initial Conditions}

Massive star formation occurs inside dense, compact clumps in giant molecular clouds $\left(\mathrm{H}_{2}\right.$ column densities are $\left.10^{23}-10^{24} \mathrm{~cm}^{-2}\right)$. Smaller mass clumps with lower peak $\mathrm{H}_{2}$ column densities do not form massive stars. Several types of molecular cloud surveys, predominantly near HII regions, have been carried out: CS-molecule surveys for dense molecular gas, 1.2-mm dust continuum surveys for massive cold dust (and hence gas) condensations, as well as $\mathrm{OH}, \mathrm{H}_{2} \mathrm{O}$, and methanol maser emission surveys for shock-excited compact regions as signposts for massive star formation. (Note that methanol maser and $\mathrm{OH}$ maser emission is exclusively associated with high-mass star formation, whereas $\mathrm{H}_{2} \mathrm{O}$ masers may also be found in low-mass star-forming regions. This is because methanol and $\mathrm{OH}$ masers are radiatively pumped and need an intense far-IR source in their vicinity; $\mathrm{H}_{2} \mathrm{O}$ masers, in contrast, are collisionally pumped in gas shocked by outflows.) These gas and dust surveys have revealed dense cold clumps (molecular hydrogen density $\mathrm{n}_{\mathrm{H}_{2}}=10^{5} \mathrm{~cm}^{-3}$, gas temperature $\mathrm{T}=10$ $20 \mathrm{~K}$, diameter $\sim 0.5 \mathrm{pc}$ ) with gas masses ranging from a few hundred to a few thousand solar masses (Plume et al. 1997, Shirley et al. 2003, Garay et al. 2004, Motte et al. 2005, Evans 2005). The methanol maser surveys at $6.7 \mathrm{GHz}$ point to hot molecular cores with internal heat sources and outflows, as well as protoclusters (Burton et al. 2005, De Buizer 2003, Minier et al. 2005).

As mentioned before, large-scale observations with the ISO, MSX, and Spitzer satellites have revealed a new class of clouds, the so-called IR dark clouds or IRDCs, and Simon et al. (2006) have identified more than 10,000 such IRDCs 
from the MSX data base. Many of these appear to be located in the $4-5 \mathrm{kpc}$ Galactic molecular ring (Jackson, private communication; see also Bronfman et al. 2000). The IRDCs are dense clouds seen in absorption against mid-IR background emission. They are mostly filamentary structures that contain condensations of cold massive cores where massive stars or even star clusters seem to form (Rathborne, Jackson \& Simon 2006). Recent mid-IR and millimetercontinuum observations show different evolutionary stages of massive star formation in adjacent cores: dense millimeter-continuum sources with and without mid-IR emission (Garay et al. 2004, their figure 4).

The origin of these structures appears to derive from supersonic turbulence in giant molecular clouds, that is, shock compression from convergent turbulent gas streams. Depending on the direction of the compression with respect to the direction of the magnetic field lines, the magnetic field will be boosted through flux freezing and hence the resulting clump will be stabilized by magnetic forces against gravitational collapse (subcritical compression). If not, the compressed clump is quickly set up for collapse (supercritical compression), in fact so quickly that the set-up time is shorter than the free-fall time. Magnetically stabilized clumps take much longer for collapse to begin, and their internal turbulent structure may make the clump prone to subfragmentation. If this is true, only supercritical compression leads to massive star formation (cf. Shu, Adams \& Lizano 1987). An interesting speculation would be that clouds without sufficiently strong magnetic fields can form lots of massive stars quasi-simultaneously, giving rise to gigantic starbursts.

\section{$2.3 \quad$ Endproducts}

\subsubsection{OB Clusters}

The endproducts of massive star formation are either dense gravitationally bound OB star clusters or loose unbound OB associations (Lada \& Lada 2003, Briceño et al. 2007). Classical examples of OB star clusters include the Orion Nebula Cluster (ONC), the dense compact cluster associated with the giant galactic HII region NGC 3603, and the R136 cluster in the 30 Dor region in the Large Magellanic Cloud (LMC; see Figure 2). These clusters roughly define 
a richness sequence in powers of 10: they contain $1,10(21)$, and $\sim 100$ massive O-type stars per cluster, with estimated total cluster masses of $10^{3}, 10^{4}$, and $10^{5} \mathrm{M}_{\odot}$, respectively (e.g., ONC: Hillenbrand 1997, Hillenbrand \& Hartmann 1998; NGC 3603: Moffat, Drissen \& Shara 1994, Drissen et al. 1995; R136: Parker \& Garmany 1993, Massey \& Hunter 1998). Although R136 can probably be considered a small young globular cluster (M. Andersen et al., submitted), there are more massive young clusters in the nearby universe, such as the very massive embedded super star cluster in the center of the NGC 5253 dwarf galaxy (Turner et al. 2003). This cluster has an ionizing flux equivalent to the presence of $4000-6000 \mathrm{O} 7 \mathrm{~V}$ stars that comes from a very compact region about $1 \mathrm{pc}$ in size, measured with the VLA. How can the formation of so many massive stars in such a small volume be possible? This question is at the heart of understanding the origin of globular clusters.

\subsubsection{OB Associations}

The classical examples of OB associations include the nearby Scorpius OB2 and Orion OB1 associations (Blaauw 1964, 1991). Another fine example of what may ultimately become an OB association is the Carina star formation complex at $2.3 \mathrm{kpc}$ (Smith \& Brooks 2007). One of the best studied extragalactic OB associations is NGC 604 in M33 (Maíz-Apellániz, Pérez \& Mas-Hesse 2004). In all these cases, the OB stars are spread over the whole face of the parent giant molecular cloud and are not densely packed at all, with distances between massive stars ranging from 1 to $10 \mathrm{pc}$. This then appears to be a completely different mode of massive star formation, although it must be noted that OB associations often contain dense clusters, too (e.g., the Carina complex harbors the well-known Trumpler 14 and 16 clusters). The question is whether OB associations are superpositions of expanded young clusters (e.g., Kroupa, Aarseth \& Hurley 2001, Bastian \& Goodwin 2006).

\subsubsection{Field OB Stars}

Do massive stars always occur in young star clusters or OB associations, or can massive stars also be found outside these regions, i.e., in the field? The answer is 
they can. It has long been realized that there exists a class of massive stars, the so-called runaway OB stars (Blaauw 1961, Poveda, Ruiz \& Allen 1967, Gies \& Bolton 1986) that are ejected from their birthplaces - clusters and associations - with velocities in excess of $40 \mathrm{kms}^{-1}$. About $10-25 \%$ of all $\mathrm{O}$ stars and about $2 \%$ of all B stars belong to this class of massive field stars. The question whether

- these runaway stars aside - other massive stars occur in the field (implying that they would be formed in isolation) has been studied by de Wit et al. (2004, 2005), following Mason et al. (1998). These authors established that 43 among the $227 \mathrm{O}$ stars brighter than eighth V-magnitude are in the field. Of these, about half can be traced back to a cluster or association origin, but about $10-20$ stars (i.e., $\sim 5-10 \%$ ) could not be assigned to any group of origin and therefore might be true field stars, born outside clusters and associations, an issue already raised in a pioneering paper by Roberts (1957). A case in point is HD93521, a high-latitude $09.5 \mathrm{~V}$ star, more than $1 \mathrm{kpc}$ above the Galactic plane, which must have formed locally in the halo (Irvine 1989)! These examples suggest that the question of the birthplaces of massive stars is not yet completely settled. The forthcoming Spitzer 8-micron imaging survey of the Magellanic Clouds (see Meixner et al. 2006) can shed new light upon the question, and in particular can pinpoint isolated massive stars in the LMC/SMC, should these objects indeed exist.

\subsection{Clues from multiplicity}

The multiplicity of massive stars is believed to be higher than that of young lowmass premain-sequence stars (Preibisch, Weigelt \& Zinnecker 2001, Duchêne et al. 2001). This means massive stars have more physical companions than low-mass stars on average. For reference, the multiplicity or, more precisely, the companion star fraction (csf) of a stellar population has been defined by Reipurth \& Zinnecker (1993) to be

$$
\operatorname{csf}=(\mathrm{B}+2 \mathrm{~T}+3 \mathrm{Q}+\ldots) /(\mathrm{S}+\mathrm{B}+\mathrm{T}+\mathrm{Q}+\ldots),
$$

where $\mathrm{S}$ is the number of single, $\mathrm{B}$ the number of binary, $\mathrm{T}$ the number of triple, and $\mathrm{Q}$ the number of quadruple systems, etc. (i.e., triple systems contribute two companions, quadruple systems three companions, etc.). For example, the 
multiplicity of the four OB stars in the Trapezium Cluster in Orion is as follows: the most massive star $\theta^{1} \mathrm{C}$ is double, the next massive star $\theta^{1} \mathrm{~A}$ is triple (a hierarchical system with a close spectroscopic binary and a wider companion), $\theta^{1} \mathrm{~B}$ is at least quadruple (kind of a Trapezium system within the Trapezium Cluster), and $\theta^{1} \mathrm{D}$ is apparently single. [Kraus et al. (2007) find some indications that $\theta^{1} \mathrm{D}$ appears extended in their speckle images. There is a fifth star in the Trapezium Cluster, $\theta^{1} \mathrm{E}$, which has recently been found to be a doublelined intermediate-mass spetroscopic binary (Herbig \& Griffin 2006, Costero et al. 2006).] Putting the number of companions of $\theta^{1} \mathrm{~A}, \mathrm{~B}, \mathrm{C}, \mathrm{D}$ into the above formula, we get $\operatorname{csf}=1.5$ at face value (probably a lower limit). This should be compared to the multiplicity of the low-mass stellar members in the Orion Nebula cluster, which is csf $=0.5$ (Padgett, Strom \& Ghez 1997; B. Reipurth et al., submitted), i.e., significantly lower.

The multiplicity statistics of other $\mathrm{OB}$ clusters, including clusters rich in $\mathrm{O}$ stars $(\mathrm{N}>5)$ and poor in $\mathrm{O}$ stars $(\mathrm{N}<3)$ has been studied by Mermilliod \& García (2001) and García \& Mermilliod (2001), with interesting results: The spectroscopic binary frequency in O-star rich clusters can vary enormously in different clusters, from $15 \%$ to $80 \%$, with no apparent correlation. If anything, there is an anticorrelation of the binary frequency and the cluster density, but this needs to be reinvestigated and confirmed. The above statistics often rely on relatively poor data; and the sampling is not complete. Recent work on higher quality data, but on a more limited number of clusters, tends to obtain a lower binary fraction in the range of $20 \%$ to $60 \%$ (Sana, Rauw \& Gosset 2005). The most dramatic example is the IC 1805 cluster where the binary frequency went down from $80 \%$ to $20 \%$ based on better data (De Becker et al. 2006).

In the O-star poor clusters almost all $\mathrm{O}$ stars are spectroscopic binaries, often double-lined and even eclipsing. These massive binaries are usually members of hierarchical triple or quadruple systems, or of trapezia, and are often located at the cluster center. The exciting star of the Orion Nebula Cluster, $\theta^{1}$ Ori $\mathrm{C}$ belongs in this category, although $\theta^{1}$ Ori $\mathrm{C}$ is not a massive close spectroscopic binary but a very eccentric visual binary, with masses of $34 \mathrm{M}_{\odot}$ $(\mathrm{O} 5.5 \mathrm{~V})$ and $15 \mathrm{M}_{\odot}(\mathrm{O} 9.5 \mathrm{~V})$ and an orbital period of about $11 \mathrm{yr}$ (Kraus et al. 2007). The orbital periods of the spectroscopic binaries in the O-star rich 
clusters are concentrated in the range of $4-5$ days, whereas in the O-star poor clusters there is a pile-up of orbital periods around $3 \pm 1$ days. In NGC 6231, for example, according to Sana (private communication), 10 out of the $16 \mathrm{O}$ stars are double-lined spectroscopic binaries: 6 with periods under 10 days (4 below 5 days); 2 with periods between 3 and 9 months, and 2 with periods of the order of a year or greater (in addition, 1 star is probably a triple-lined spectroscopic binary). The luminosity ratios are all in the range $1-10$, otherwise one would not detect them as double-lined spectroscopic binaries. This implies that the secondaries are probably early B stars, and the primary-to-secondary mass ratios must be about 3 (at maximum) or lower. It is also worth mentioning that there is no very short-period highly eccentric $\mathrm{O}+\mathrm{O}$ binary known at this time (Sana, private communication). These fascinating and surprising facts challenge our views of massive star formation and provide clues to their origin, clues too complex to fully decipher yet but hinting at gravitational dynamics playing a role - beyond mere disk or filament fragmentation (see Section 5, where the formation of binary and multiple systems is extensively discussed; see also Zinnecker 2003).

Of course, it is equally important to study the multiplicity of massive stars in OB associations where the stellar density is much lower than in OB clusters, and dynamical interactions between the forming massive stars should be less of an issue. For example, the Orion OB1 association contains $\sim 70$ massive stars in its three subgroups 1a,b,c (subgroup 1d is the Orion Nebula Cluster). Of these 70 OB stars, $20 \%$ are spectroscopic binaries with periods less than 10 days (Morrell \& Levato 1991). The three subgroups show variations in their spectroscopic binary fractions: subgroup 1a is average, subgroup $1 \mathrm{~b}$ is a factor 1.5 above the average, and subgroup $1 \mathrm{c}$ is a factor 1.5 below the average. The situation in the nearby Scorpius-Centaurus OB2 association is as follows: Among the 48 early B stars (B05 to B3V) there are 25 binaries, and 20 of them are spectroscopic binaries with known periods in the range of $0.9-34.2$ days, with a median of 5.7 days (Brown 2001).

In conclusion, it seems that the spectroscopic binary fraction among massive stars in $\mathrm{OB}$ associations is surprisingly similar to that in $\mathrm{OB}$ clusters rich in $\mathrm{O}$ stars (about $40 \%$ on average), and the fraction of very close spectroscopic and 
eclipsing massive binaries in $\mathrm{OB}$ associations with orbital periods below 5 10 days (about $20 \%$, judging from the Orion and Scorpius-Centaurus regions) is a factor of two lower than in $\mathrm{OB}$ clusters. Thus OB clusters appear to contain more of a population of very tight (hard) binaries, possibly an effect owing to dynamical encounters after birth; this is an effect that is absent in OB associations.

The future of spectroscopic massive binary research lies in the near-IR and in multiepoch radial velocity surveys of embedded massive stars. First results (and successes) have been reported by Apai et al. (2007), indicating that massive close binaries indeed form at a very early stage.

\subsection{Upper Initial Mass Function and Upper Mass Limit}

The mass distribution function of massive stars at birth (the so-called Initial Mass Function or IMF for short) is a complicated matter, because (a) massive stars quickly lose some of their initial mass through stellar winds, (b) many of these massive stars are unresolved binaries, and $(c)$ massive stars tend to be born in the centers of $\mathrm{OB}$ clusters or tend to sink preferentially toward the cluster center, leaving behind their lower mass siblings that live in the cluster outskirts. This introduces a bias into the mass distribution, flattening a power-law slope. The upper IMF from about 10 to $100 \mathrm{M}_{\odot}$ is usually found to be a universal power law, with logarithmic slope -1.35, first found by Salpeter (1955) for a range of masses below $10 \mathrm{M}_{\odot}$. We refer here to the early observational work of Garmany, Conti \& Chiosi (1982) and the summary of Massey (1998). The implication of a Salpeter slope or other similar slopes of the IMF for the number of stars born in different mass intervals (for convenience spaced by a factor of two) can be seen in Table 2, which has been normalized to contain exactly one object in the highest mass interval. This is instructive, because it shows dramatically how rare the O/WR-type massive stars (interval $64-128 \mathrm{M}_{\odot}$ ) are compared with the early B-type massive stars $\left(8-16 \mathrm{M}_{\odot}\right)$, or with the solar-type low-mass stars $\left(1-2 \mathrm{M}_{\odot}\right)$; see Zinnecker (1996).

The IMF of massive stars in the aforementioned OB clusters (Orion Nebula Cluster, NGC 3603, and R136 in 30 Dor) is discussed by Pflamm-Altenburg \& 
Table 2: Initial Mass Function $\left(\mathrm{dN} / \mathrm{d} \log \mathrm{M} \sim \mathrm{M}^{-\mathrm{x}}\right)$ examples

\begin{tabular}{cccc}
\hline \hline Mass range & \multicolumn{3}{c}{ Logarithmic slope } \\
\hline $0.5-1 \mathrm{M}_{\odot}$ & $\mathrm{x}=1$ & $\mathrm{x}=1.35$ & $\mathrm{x}=1.7$ \\
$1-2 \mathrm{M}_{\odot}$ & 128 & 700 & 3822 \\
$2-4 \mathrm{M}_{\odot}$ & 64 & 275 & 1176 \\
$4-8 \mathrm{M}_{\odot}$ & 32 & 108 & 362 \\
$8-16 \mathrm{M}_{\odot}$ & 16 & 42 & 111 \\
$16-32 \mathrm{M}_{\odot}$ & 8 & 16.6 & 34.3 \\
$32-64 \mathrm{M}_{\odot}$ & 4 & 6.5 & 10.6 \\
$64-128 \mathrm{M}_{\odot}$ & 2 & 2.55 & 3.25 \\
\hline
\end{tabular}

Kroupa (2006), Stolte et al. (2006), and Massey \& Hunter (1998), respectively. [Other studies of the Orion Nebula Cluster include Zinnecker, McCaughrean \& Wilking (1993); Hillenbrand \& Hartmann (1998). The stellar content in the biggest galactic HII region NGC 3603 was also investigated by Drissen et al. (1995); Hofmann, Seggewiss \& Weigelt (1995); Eisenhauer et al. (1998); Brandl et al. (1999); Moffat et al. (2004); and Sung \& Bessell (2004). An important investigation of the IMF of R136 is that of Sirianni et al. (2000).] Whereas Pflamm-Altenburg \& Kroupa (2006) find a deficit of high-mass stars in the Orion cluster, Stolte et al. (2006) derive an excess of massive stars in the NGC 3603 cluster core, reflected by a power-law slope of -0.9 (probably due to mass segregation). It is only in R136 that the power-law slope is almost exactly the same as the Salpeter value. It is worth noting here that the slope of the mass function of high-mass stars in the range of $8-40 \mathrm{M}_{\odot}$ in the wider field in the 30 Dor region is apparently the same as in the R136/NGC 2070 cluster (Selman $\&$ Melnick 2005). The latter authors do not find the much steeper slope of the IMF (for the range of $25 \mathrm{M}_{\odot}$ to $120 \mathrm{M}_{\odot}$ ) derived by Massey (2002) for the global OB field population in the LMC. They suspect that selective incompleteness at $\mathrm{V}=12$ owing to detector saturation and Be star contamination lies at the origin of this discrepancy. 
The question of the IMF of massive stars in OB associations was discussed long ago by Garmany, Conti \& Massey (1980) and later by Massey, Johnson \& Degioia-Eastwood (1995). They concluded that the IMF is normal, i.e., consistent with a Salpeter power-law. A fine discussion of upper IMF slopes in various young clusters and associations including all the caveats and selection effects was given by Scalo (1998). He noted that individual realizations of IMF slopes can vary, but the average slope is indeed close to the Salpeter value -1.35.

The question of whether there is a physical (rather than statistical) upper mass end to the IMF is of great interest to anyone interested in population synthesis, galactic evolution, and cosmology. For example, if the stellar upper mass limit were $120 \mathrm{M}_{\odot}$, pair instability supernovae requiring stellar masses at the time of explosion between 140 and $260 \mathrm{M}_{\odot}$ (Heger et al. 2003) could not happen. Larson (1982) originally asked the observational question whether there was a correlation between the mass of a molecular cloud and the maximum mass of a star that could form in it. His result was that indeed the maximum stellar mass scaled with the mass of the parent cloud, roughly with its square root. It takes a giant molecular cloud of $10^{5} \mathrm{M}_{\odot}$ to form a $50 \mathrm{M}_{\odot}$ star; a cloud of $10^{3} \mathrm{M}_{\odot}$ can only spawn a maximum stellar mass of $8 \mathrm{M}_{\odot}$. The implication is that massive stars form in clouds of mass between $10^{3}$ and $10^{5} \mathrm{M}_{\odot}$ or more, probably because only these have sufficiently massive substructure (clumps).

Weidner \& Kroupa (2004) and Figer (2005) discussed the upper limit to the masses of stars, based on observations of the R136 cluster in the LMC and the Arches cluster near the Galactic Center, respectively. They pointed out that these clusters are so massive that given a Salpeter IMF one would expect to find stars as massive as $750 \mathrm{M}_{\odot}$ and $500 \mathrm{M}_{\odot}$, respectively, whereas the most massive stars seen do not exceed $140 \mathrm{M}_{\odot}$ and $130 \mathrm{M}_{\odot}$, respectively. This suggests a firm upper mass limit of $150 \mathrm{M}_{\odot}$. Otherwise, a sharp down-turn of the IMF near $150 \mathrm{M}_{\odot}$ would be required (see the extensive discussion in Elmegreen 2000). Or the very massive stars have already exploded/imploded during the dust-obscured, hidden, early evolutionary stages - an unlikely scenario. Oey \& Clarke (2005) also gave a statistical confirmation of a stellar upper mass limit around $120-200 \mathrm{M}_{\odot}$, if the IMF is Salpeter-like. Koen (2006) further analyzed the upper IMF in the R136 cluster with two different statistical techniques and 
suggested an upper mass limit of $140-160 \mathrm{M}_{\odot}$. Thus all four studies agree on the existence of a physical upper limit in the stellar mass distribution.

\subsection{Feedback and Triggering}

This topic deserves its own review. The question we ask here is the following: What does the energy and momentum input of massive stars in terms of expanding HII regions, stellar winds, or supernova shock waves do to the parent clouds? Is the cloud primarily disrupted or is new star formation triggered? Which of the above agents (HII regions, stellar winds, or supernova shock waves) provides the best trigger for new OB star formation and for new low-mass star formation? We are only beginning to answer these questions.

A key observation in this context is the fact that the high-mass and low-mass stellar populations in the subgroups of $\mathrm{OB}$ associations appear to be coeval, i.e., the nuclear age of the massive stars is the same as that of the lower mass premain-sequence objects (Preibisch \& Zinnecker 1999, 2007; Briceño et al. 2007). This would appear to require a fast, coherent trigger, such as a supernova shock wave. Indeed, in the Scorpius-Centaurus association there is evidence that the shock wave of a supernova in one OB subgroup triggered the formation of another subgroup (de Geus 1992). However, there is also other evidence that radiation from massive stars [by a process called radiative implosion (e.g., Kessel-Deynet \& Burkert 2003)] can only trigger the formation of low- and intermediate-mass objects (Lee \& Chen 2007 and references therein).

The classic theory of triggered massive star formation is the one by Elmegreen \& Lada (1977). In their theory, an ionization shock front provides the pressure on an adjacent layer of molecular gas to compress it and heat it, thus stimulating gravitational instability of massive gas layers, hence the formation of massive stars - and perhaps only massive stars. Low-mass star formation may not be triggered with this mechanism. If true, this would produce an anomalous IMF with only massive stars (known as bimodal star formation, cf. Güsten \& Mezger 1982). It is worth noting that such characteristics - small groups of massive stars only - may be needed for the decay of small N-body systems that give rise to the dynamical ejection of runaway OB stars (Clarke \& Pringle 1992). 


\section{MASSIVE STAR FORMATION: BASIC THEORY}

\subsection{Sequence of Events}

Starting from a pre-existing giant molecular cloud, the sequence of events is likely as follows:

1. Formation of cold dense molecular cores or filaments, induced by gravoturbulent cloud fragmentation (Mac Low \& Klessen 2004). This means that supersonic turbulence rapidly produces localized compressed pockets of gas, some of which remain gravitationally bound and provide the initial conditions for collapse (Padoan \& Nordlund 2002; Klessen et al. 2005). A characteristic density of about $10^{5} \mathrm{~cm}^{-3}$ and temperature of $10-15 \mathrm{~K}$ results from the equation of state of dusty molecular gas of solar abundance (Jappsen et al. 2005, Larson 2005).

2. Nonhomologous gravitational collapse of portions of the cores into optically thick, pressure-supported protostellar embryos with initial masses of the order of $10^{-3} \mathrm{M}_{\odot}$ (Larson 1969, Bate 2000). The term nonhomologous collapse refers to the fact that the relative distribution of material changes, as opposed to a homologous or self-similar collapse. (This is different from Shu's (1977) inside-out collapse of a self-similar isothermal sphere).

3. Accretion of material onto protostellar objects as they evolve toward the main sequence. For low-mass objects the accretion stops well before hydrogen burning commences. These premain-sequence objects of fixed mass then slowly and quasi-hydrostatically contract to the main sequence (Palla \& Stahler 1993, Baraffe et al. 2002). However, high-mass objects eventually start burning hydrogen and develop radiation-driven winds as they continue to accrete and evolve up the main sequence to hotter and more luminous states (Kudritzki 2002).

4. Disruption of the birth cloud, as the first high-mass stars strongly influence their environment by their winds, outflows, and UV radiation, and 
eventually become supernovae. The most massive stars go supernova after $\sim 3$ Myr. When the remnant molecular cloud has been dissipated, the result is mostly a cluster of $\mathrm{OB}$ stars or an $\mathrm{OB}$ association, with an associated cospatial population of lower mass stars (Zinnecker, McCaughrean \& Wilking 1993). Often, several evolutionary stages of star formation can be found side by side; the 30 Dor region in the LMC is a good example (Walborn et al. 1999).

We shall denote these four phases as the compression, collapse, accretion, and disruption phases of high-mass star formation, respectively. All phases can occur simultaneously and side-by-side in a molecular cloud. Below, we give a fairly detailed yet still greatly simplified discussion of these phases, including protostellar luminosity and ionization evolution as a function of growing stellar mass.

\subsection{The Compression Phase}

This first step toward high-mass star formation is either a starless core $\left(\sim 100 \mathrm{M}_{\odot}\right)$ or a starless clump $\left(\sim 1000_{\odot}\right)$ of molecular gas in a giant molecular cloud. McKee \& Tan (2003) envisage that these cores are molecular condensations in a turbulence-supported quasi-equilibrium that ultimately form single or gravitationally bound multiple massive protostars. As these authors argue, turbulent and pressurized clouds permit sufficient material to be available in the cores of giant molecular clouds for high-mass star formation. Mechanical energy must be continuously injected into the clump in order to maintain this quasi-equilibrium between turbulence and gravity. The assumption is that this energy is either injected from within the cores from the kinetic energy of outflows and accretion shocks, or it comes from the outside and cascades down to smaller scale sizes. It is furthermore assumed that (small-scale) turbulence acts as an isotropic pressure.

In an alternate scenario by Bonnell et al. (1997, 2001a), the compression phase is more of a transient phase due to the random motions in the selfgravitating cloud. Smoothed particle hydrodynamics (SPH, see the sidebar Smoothed Particle Hydrodynamics with Sink Particles) simulations of this phase 
show sheets, filaments, and cores forming (see Figure 3). Some cores collapse and fragment, marking the beginning of the collapse phase for these objects, while in other parts of the cloud compression is still occurring.

The philosophical difference between the approach of McKee \& Tan (2003) and that of Bonnell et al. (1997, 2001a) can be summarized as "monolithic collapse" versus "competitive accretion." For the former, the mass necessary for massive star formation is intimately associated with the final product. If there are bulk motions of the embryo star, its protostellar core participates in those motions. The only competition for the infalling material is between close members of a multiple system. For competitive accretion, the material that makes up a particular star can come from various parts of the parent cloud. Protostars move relative to the molecular gas; the only gas that is intimately associated with any particular protostar is in its circumstellar disk and envelope. Because multiple protostars are often formed together at the same time, each protostar competes for the available molecular material (see Section 4.2).

Crutcher's $(1999,2005)$ summary of the available Zeeman measurements of magnetic field strengths in molecular clouds suggests that magnetic fields likely play an important role in molecular cloud dynamics. Vázquez-Semadeni et al. (2005) studied the evolution of clumps and cores formed as turbulent density fluctuations in nearly isothermal molecular clouds, considering both the magnetic and nonmagnetic cases with driven turbulence. In the nonmagnetic case the authors find that the cores are unlikely to reach a hydrostatic state - necessary for monolithic collapse - if the molecular clouds have an effective polytropic exponent less than $4 / 3$. In this case, cores are transient, either proceeding directly to collapse or re-expanding on a dynamical timescale.

The magnetically subcritical clouds simulated by Vázquez-Semadeni et al. (2005) do not produce magnetostatic clumps, but rather a few marginally bound clumps that are subsequently dispersed. Ambipolar diffusion - had it been included in the simulations - could have increased the clumps' likelihood to become bound and subsequently to collapse. For clouds with weaker magnetic fields a few cores form and collapse on a timescale slightly larger than the cloud's free-fall timescale. In their most supercritical simulation, fewer clumps and cores form than in their nonmagnetic counterpart, and these cores reexpand 
because they are not Jeans unstable. The authors thus conclude that not all cores observed in molecular clouds will necessarily form stars and that magnetic fields may help reduce the star-formation efficiency by reducing core formation rather than by delaying or inhibiting the collapse of individual cores.

\section{Smoothed Particle Hydrodynamics with Sink Particles}

Many simulations of star formation in turbulent interstellar clouds are conducted using a Lagrangian method to solve the equations of hydrodynamics. This is called smoothed particle hydrodynamics (SPH). It is a 3D method that assumes no symmetries and uses no imposed grids. Monaghan (2005) gives a recent review of the method and its applications (see also Monaghan 1992). With $\mathrm{SPH}$, the gas is represented by a number of sampling points ("particles"), each associated with a certain gas mass. The mass of each particle is distributed in space according to a spread function so that density and other hydrodynamic quantities are continuous in space. The particles are allowed to move in the computational domain under the laws of self-gravitating hydrodynamics (which are evaluated at the particle positions). The system is evolved in time using a standard integration routine.

The timestep with which the simulations evolve is chosen such that all changes to the system's state are examined at the appropriate speed. For example, during the collapse phase, when infalling particles approach each other, the timestep gets progressively shorter. This may potentially bring the simulations to an early halt as the timestep converges to zero. This problem is overcome with the introduction of star (sink) particles of finite radii that each replace groups of dense neighboring gas particles above a certain density threshold (see Bate, Bonnell \& Price 1995). The sink particles are then evolved as

collisionless matter that can accrete from the lower density gas whenever gas particles approach within the sink radius. The final state of the system in such simulations involves groups of sink particles in spatial distributions resembling observed young clusters. However, all information on scales smaller than the sink radius is lost; such sink particles cannot later become sources of outflows, unless specifically instructed to do so. Note that sink particles are also usefully employed in Eulerian collapse calculations, as shown by Krumholz, McKee \& Klein (2004). 
In order for fragmentation to be realistically modelled, the numerical resolution of the SPH simulations must be such that they obey the Jeans condition. This states that "the local Jeans mass must always remain resolved," i.e., it must contain more than a certain number of particles (Truelove et al. 1997, Bate \& Burkert 1997, Kitsionas \& Whitworth 2002, Martel, Evans \& Shapiro 2006). The Jeans mass decreases as the density increases up to the point at which the gas stops being approximately isothermal and begins to heat up adiabatically. From there on, the Jeans mass starts increasing again with density. The density at which this occurs defines the minimum Jeans mass that the simulation has to resolve and, thus, the minimum number of particles required. Present-day computer capabilities allow the use of several million SPH particles per simulation, a number that is nominally adequate for resolving the density at which adiabatic heating switches on. Using parallel computing facilities presently available, such computations take several months for each evolutionary model.

The assumption of driven as opposed to decaying turbulence is important in these and similar simulations (cf. Heitsch, Mac Low \& Klessen 2001, Li \& Nakamura 2006).

Indeed, Li \& Nakamura (2006) using the best 3D MHD simulation technique to date have shown that initial turbulence in a cluster forming region is quickly replaced by motions generated by protostellar outflows. This protostellar outflow-driven turbulence can keep a protocluster clump close to virial equilibrium long after the initial turbulence has decayed away. This may explain the early molecular cloud observations of Bertoldi \& McKee (1992) and lend support to the recent equilibrium cluster formation models of Tan, Krumholz \& McKee (2006). It could even imply that the stellar IMF is regulated by outflow feedback (Silk 1995, Adams \& Fatuzzo 1996). However, the ultimate test of these predictions has not yet been made and requires much more observational work. At the same time, competing, more violent cluster formation models have been proposed in the literature (e.g. Bonnell, Bate \& Vine 2003), and the jury is still out. Essentially, the question is whether star formation is slow or fast (Ballesteros-Paredes, Hartmann \& Vázquez-Semadeni 1999, Glover \& Mac Low 2007). 


\subsection{The Collapse Phase}

Gravity plays the dominant role in star formation. To form a star, gravity must overcome pressure, magnetic forces, internal turbulence, and rotation. In the simplest case of gravity versus gas pressure, one defines the Jeans mass,

$$
\mathrm{M}_{\text {Jeans }} \simeq 1.1 \mathrm{M}_{\odot}\left[\frac{\mathrm{T}_{\text {gas }}}{10 \mathrm{~K}}\right]^{3 / 2}\left[\frac{\rho}{10^{-19} \mathrm{~g} \mathrm{~cm}^{-3}}\right]^{-1 / 2}
$$

as the smallest mass for which gravity can become dominant. The normalization is consistent with typical initial conditions.

Turbulence as a repulsive force will exceed gas pressure if motions are supersonic. Unless continually replenished, however, supersonic turbulence dies out on a dynamical timescale (see, e.g., Stone, Ostriker \& Gammie 1998; Clark \& Bonnell 2005; Kritsuk, Norman \& Padoan 2006; for a summary of earlier work, see Mac Low \& Klessen 2004).

As Shu, Adams \& Lizano (1987) point out, once gravity dominates pressure and magnetic forces in an optically thin gas capable of radiating compressional heat, it remains dominant and the gas collapses on a free-fall timescale

$$
\mathrm{t}_{\mathrm{ff}} \simeq 2.1 \times 10^{5} \mathrm{yr}\left[\frac{\rho}{10^{-19} \mathrm{~g} \mathrm{~cm}^{-3}}\right]^{-1 / 2} .
$$

That is, the densest parts collapse the fastest, and the Jeans mass decreases during collapse. The gas collapses nonhomologously until the densest parts become optically thick, allowing the gas to heat up adiabatically and to increase the gas pressure dramatically. Rotational (centrifugal) forces increase during gravitational collapse owing to conservation of angular momentum, so flattened structures and accretion disks (e.g., Black \& Bodenheimer 1975; Terebey, Shu \& Cassen 1984; Yorke \& Bodenheimer 1999) are expected phenomena of gravitational collapse.

\subsection{The Accretion Phase}

\subsubsection{Formation of the Hydrostatic Core}

The formation of low-mass stars is explained by the nonhomologous collapse of a slowly rotating fragment of molecular material; the collapse is stopped 
in the central regions when the object becomes optically thick (Larson 1969, Woodward 1978, Winkler \& Newman 1980, Masunaga, Miyama \& Inutsuka 1998; for a more recent review, see Klein, Fisher \& McKee 2004). There is a second, inside-out collapse in the center for $\mathrm{T}_{\text {gas }} \simeq 2000 \mathrm{~K}$ when molecular hydrogen dissociates. When the second core is optically thick and thermally ionized, pressure forces are able to balance gravity on a dynamical timescale, and one speaks of an accreting quasi-hydrostatic core as more material rains down. When it forms, the second hydrostatic core contains somewhat more than a Jupiter mass and has a radius on the order of $(3-5) \mathrm{R}_{\odot}$ (Winkler \& Newman 1980, Masunaga \& Inutsuka 2000).

As long as material continues to flow onto the quasi-hydrostatic core, the core grows in mass. Simultaneously, it contracts on a thermal Kelvin-Helmholtz timescale $\tau_{\mathrm{KH}} \sim \mathrm{GM}_{*}^{2} / \mathrm{R}_{*} \mathrm{~L}_{*}$ toward hydrogen-burning densities and temperatures. Figure 4 shows the Kelvin-Helmholtz timescale to the ZAMS as a function of stellar mass. Except for the growth of mass and angular momentum, the interior regions of the hydrostatic core are generally ignorant of and not influenced by the details of the accretion process. This allows us to separate the problem of star formation into several distinct parts: $(a)$ the evolution of the central core, $(b)$ the details of transporting material from the disk onto the core, $(c)$ transporting material inward within the disk, and $(d)$ accretion onto the disk. Fundamental differences between low-mass and high-mass star formation can be attributed to differences in the above processes and, in particular, to significant differences in the timescales involved and in the local radiative environment.

\subsubsection{Evolution of Accreting Cores}

Accretion onto the (proto)star will affect the core's outer (atmosphere) regions and the overall spectral appearance. We estimate the thickness $\Delta \mathrm{R}_{\mathrm{dyn}}$ of the outer stellar regions that are dynamically affected by accretion to be:

$$
\frac{\Delta \mathrm{R}_{\mathrm{dyn}}}{\mathrm{R}_{*}} \sim \frac{\dot{\mathrm{M}}_{\mathrm{S}-\mathrm{acc}} \mathrm{t}_{\mathrm{S}-\mathrm{dyn}}}{\mathrm{M}_{*}}=\frac{\mathrm{t}_{\mathrm{S}-\mathrm{dyn}}}{\mathrm{t}_{\mathrm{S}-\mathrm{acc}}}
$$

where $\mathrm{t}_{\mathrm{S}-\mathrm{dyn}} \simeq \mathrm{R}_{*} / \mathrm{v}_{\mathrm{esc}}$, the stellar dynamical timescale, is the time for an acoustic wave to cross through the core or the orbital period of a body just above 
the core's surface (generally less than a day). Because $\mathrm{t}_{\mathrm{S}-\mathrm{dyn}}<<<\mathrm{t}_{\mathrm{S}-\mathrm{acc}}=$ $\mathrm{M}_{*} / \dot{\mathrm{M}}_{\mathrm{S}-\text { acc }}$ (the accretion timescale), $\Delta \mathrm{R}_{\text {dyn }} / \mathrm{R}_{*}$ is extremely small $\left(<10^{-5}\right.$ for accretion rates $\dot{\mathrm{M}}_{\mathrm{S}-\mathrm{acc}}<10^{-2} \mathrm{M}_{\odot} \mathrm{yr}^{-1}$ ).

It is not easy to estimate the size of the region thermally affected by the accretion flow onto the (proto)star, because the radiation transfer in an optically thick plasma depends on details of the complex accretion geometry. Exactly how material flows from the disk onto the (proto)star remains an unsolved theoretical problem.

One can expect a (proto)star's location in the Hertzsprung-Russell (HR) diagram to be strongly affected by accretion whenever the Kelvin-Helmholtz timescale $\tau_{\mathrm{KH}}$ exceeds the accretion timescale $\tau_{\mathrm{acc}}$. However, even when this criterion is not fulfilled, the Kelvin-Helmholtz timescale for the outer regions of a star can locally exceed the accretion timescale, and accretion can affect the radius, effective temperature, and luminosity of the star (Kippenhahn \& Meyer-Hofmeister 1975).

Kippenhahn \& Meyer-Hofmeister (1975) consider the case of mass transfer within a close binary system. At high accretion rates $\dot{\mathrm{M}}_{\mathrm{S}-\mathrm{acc}} \gtrsim 10^{-3} \mathrm{M}_{\odot} \mathrm{yr}^{-1}$, accreting main-sequence stars of mass $\mathrm{M}_{*} \gtrsim 5 \mathrm{M}_{\odot}$ bloat up to radii exceeding 10 times their ZAMS values. Although not strictly applicable to the case of accreting premain-sequence stars, one can expect the same qualitative effect of high accretion rates in this mass range. These results demonstrate the importance of accretion for the appearance of the central hydrostatic cores.

For low-mass stars, it is generally accepted that material is transported onto the central core through an accretion disk (e.g., Shu, Adams \& Lizano 1987). The net gain per unit time of gravitational potential energy of the accreted material $\mathrm{GM}_{*} \dot{\mathrm{M}}_{\mathrm{S}-\mathrm{acc}} / \mathrm{R}_{*}$ is partly converted into rotational energy of the core and disk $(\sim 1 / 4)$ and partially converted into heat $(\sim 3 / 4)$, which is radiated away (Yorke \& Krügel 1977). Part of the gravitational energy is converted into heat in a series of disk accretion shocks (Yorke \& Bodenheimer 1999); part of it is converted into heat within the disk by the same viscous processes that transport angular momentum outward and allow the radial flow of material inward; and part of it is converted into heat in the accretion flow and shocks/relaxation zones on the (proto)stellar surface. 
As mentioned above, the details of how the gas is ultimately transported from the disk onto the core are still unclear. For low-mass stars it has been postulated that magnetically focused flows (Blandford \& Payne 1982) and/or accretion columns (Königl 1991, Edwards et al. 1993) and/or X-winds (Shu et al. 1994, 1995) are involved. Because $50 \%\left(\simeq 3 / 8 \mathrm{GM}_{*} \dot{\mathrm{M}}_{\mathrm{S}-\mathrm{acc}} / \mathrm{R}_{*}\right)$ of the total gravitational energy is converted into heat and radiated away within $1 R_{*}$ of the core, one can expect higher temperatures and luminosities as either $\mathrm{M}_{*}$ or $\dot{\mathrm{M}}_{\mathrm{S}-\mathrm{acc}}$ increases or as the core contracts. The temperature of this hot shocked gas

$$
\mathrm{T} \simeq \frac{\mathrm{GM}_{*} / \mathrm{R}_{*}}{\mathrm{k} / \mu \mathrm{m}_{\mathrm{H}}} \simeq 10^{6} \mathrm{~K}\left[\frac{\mathrm{M}_{*}}{1 \mathrm{M}_{\odot}}\right]\left[\frac{\mathrm{R}_{*}}{10 \mathrm{R}_{\odot}}\right]^{-1}
$$

is sufficiently high to produce X-rays even for low-mass stars.

The above argument is only slightly modified when the core and disk produce a wind or outflow. We then speak of a net mass accretion of $\dot{\mathrm{M}}_{\mathrm{S}-\mathrm{acc}}-\dot{\mathrm{M}}_{\mathrm{S}-\text { wind }}$. A portion of the core's rotational energy and angular momentum can be converted into kinetic energy and angular momentum of the wind.

A necessary condition to accrete sufficient material to produce a massive star is thus,

$$
\mathrm{M}_{*}=\int_{0}^{\mathrm{t}}\left[\dot{\mathrm{M}}_{\mathrm{S}-\mathrm{acc}}\left(\mathrm{t}^{\prime}\right)-\dot{\mathrm{M}}_{\mathrm{S}-\text { wind }}\left(\mathrm{t}^{\prime}\right)\right] \mathrm{dt}^{\prime} \gtrsim 8 \mathrm{M}_{\odot} .
$$

That is, the accretion rate $\dot{\mathrm{M}}_{\mathrm{S}-\text { acc }}$ onto an embryo object must exceed the outflow rate $\dot{\mathrm{M}}_{\mathrm{S}-\text { wind }}$ during a significant proportion of the formation process. For this to occur, the acceleration owing to gravity must exceed the outward directed radiative acceleration of the accreting core. Whereas gravity $\mathrm{GM}_{*} / \mathrm{r}^{2}$ at each radial point in an envelope increases linearly with core mass, the radiative acceleration of dusty material $\kappa \mathrm{L} / 4 \pi \mathrm{r}^{2} \mathrm{c}$ is proportional to the core's luminosity. This increases as a high power of stellar mass. A lower limit to the core's luminosity is the ZAMS luminosity (see Figure 4).

Thus, to allow infall, we require as a necessary condition

$$
\kappa_{\mathrm{eff}} \mathrm{L} / 4 \pi \mathrm{r}^{2} \mathrm{c}<\mathrm{GM}_{*} / \mathrm{r}^{2}
$$

with $\mathrm{L}=\mathrm{L}_{*}+\mathrm{L}_{\text {acc }}$, which translates into

$$
\kappa_{\mathrm{eff}}<130 \mathrm{~cm}^{2} \mathrm{~g}^{-1}\left[\frac{\mathrm{M}_{*}}{10 \mathrm{M}_{\odot}}\right]\left[\frac{\mathrm{L}}{1000 \mathrm{~L}_{\odot}}\right]^{-1},
$$


where the effective opacity for radiative acceleration of accretable material is defined as

$$
\kappa_{\text {eff }}=\int_{0}^{\infty} \kappa_{\nu}^{\mathrm{rad}} \mathrm{F}_{\nu} \mathrm{d} \nu / \mathrm{F},
$$

here $\kappa_{\nu}^{\mathrm{rad}}$ is the frequency-dependent gram-opacity (cgs-units: $\mathrm{cm}^{2} \mathrm{~g}^{-1}$ ) of the material subject to radiative acceleration and $\mathrm{F}=\int_{0}^{\infty} \mathrm{F}_{\nu} \mathrm{d} \nu$ is the radiative flux. (For a more complete discussion of the definition of $\kappa_{\nu}^{\text {rad }}$, including effects of nonisotropic scattering and photoejection of particles from the dust, see Yorke 1988.) The (proto)star's luminosity is given by the sum of its intrinsic luminosity $\mathrm{L}_{*}$ and the luminosity $\mathrm{L}_{\mathrm{acc}}$ emitted by the dissipation of kinetic energy of the material being accreted.

For dusty gas, $\kappa_{\nu}^{\mathrm{rad}}$ is strongly frequency-dependent. Depending on the hardness of radiation emitted by the central source and the accretion shocks, dusty gas could be repelled from the star. The ISM extinction in the J-band, for instance, corresponds to $\kappa=130 \mathrm{~cm}^{2} \mathrm{~g}^{-1}$. In Figure 5 we indicate values of $\kappa_{\text {eff }}$ for dusty gas at solar abundances $\left(\kappa_{\nu}^{\text {rad }}\right.$ taken from the Preibisch et al. 1993 dust model with and without ice-coated grains) illuminated by $600 \mathrm{~K}$ and $6000 \mathrm{~K}$ black bodies [i.e., $\mathrm{F}_{\nu} \propto \mathrm{B}_{\nu}\left(\mathrm{T}_{*}\right)$ ], respectively, and for a fully ionized dustless hydrogen plasma.

Figure 6 displays the mean effective opacity of dusty gas as defined by Equation 4, using the Preibisch et al. (1993) dust model with and without ice-coated grains (blue and red curves, respectively) and assuming blackbody radiation at the temperature $T_{\text {rad }}$. Using the mass scale on the right of Figure 6 we note that the net force on dusty gas surrounding a deeply embedded $100 \mathrm{M}_{\odot}$ main-sequence star is directed toward the star as long as the star appears to the dust to be a $\lesssim 50-\mathrm{K}$ source. By contrast, for an unobscured $3 \mathrm{M}_{\odot}$ mainsequence star with $\mathrm{T}_{\text {eff }} \gtrsim 10^{4} \mathrm{~K}$ the net force on dusty gas would be directed away from the star.

\subsection{Overcoming Radiative Acceleration}

Next we discuss how Equation 2 can be satisfied, i.e., accretional growth of an already existing stellar embryo can be enabled. At least one of the following conditions must be met: $(a) \kappa_{\text {eff }}$ must be sufficiently low, i.e., significantly lower 
than its ISM value for optical/UV radiation; (b) the total luminosity L must be reduced; or $(c)$ gravity (i.e., the stellar mass $\mathrm{M}_{*}$ ) must be increased. Below, we discuss each of these three possibilities.

\subsubsection{Reduce $\kappa_{\text {eff }}$}

Owing to the strong frequency dependence of dust opacity, $\kappa_{\text {eff }}$ can be significantly lower than its ISM value if the radiation field seen by the accreting material is that of a cold, embedded object. When embedded the protostellar radiation field is shifted from the optical/UV - where the dust absorbs the photons - into the far-IR where the dust reemits the absorbed energy. Alternatively, $\kappa_{\text {eff }}$ can also be reduced if the average size of dust grains increases (but remains compact rather than becoming fractal) or if most of the dust is destroyed. In their pioneering efforts, Kahn (1974) and Wolfire \& Cassinelli (1987) studied the 1D, spherically symmetric accretion problem for massive star formation with an emphasis on the dust opacity. Kahn concluded that a 40$\mathrm{M}_{\odot}$ star could be formed by spherically symmetric accretion, but his assumed dust destruction temperature $\mathrm{T}_{\mathrm{sub}}=3600 \mathrm{~K}$ is too high by a factor of about two (implying for the outer regions greater protection from radiation pressure). Furthermore, his assumed grain opacities, which can be written in the form $\kappa \simeq 100 \mathrm{~cm}^{2} \mathrm{~g}^{-1}\left(\mathrm{~T}_{\text {dust }} / 9000 \mathrm{~K}\right)^{2}$, were somewhat too low.

In a more careful treatment of the dust (but still assuming steady-state spherically symmetric infall), Wolfire \& Cassinelli (1987) concluded that very massive stars can form only if the dust has been significantly modified. By contrast, Yorke \& Krügel (1977) showed in a hydrodynamical simulation that spherically symmetric accretion must be nonsteady for the high-mass case. They were able to produce stars of masses $17 \mathrm{M}_{\odot}$ and $36 \mathrm{M}_{\odot}$ from clouds of masses $50 \mathrm{M}_{\odot}$ and $150 \mathrm{M}_{\odot}$, respectively, in a highly variable accretion flow.

These early attempts to explain high-mass star formation suffer significantly from the fact that spherically symmetric infall was assumed.

The effective opacity of the accreting material can also be reduced by density inhomogeneities resulting from the photon bubble instability. The radiation escapes readily through the gaps between the shocks that are driven by distur- 
bances in the radiation flux (Turner, Quataert \& Yorke 2007). Another possibile explanation for reducing the effective opacity is the accretion of optically thick blobs or fingers. These can be expected to form via Rayleigh-Taylor instabilities in the radiation-inflated cavities produced by luminous (proto)stars (Krumholz, McKee \& Klein 2005a; Yorke 2002). In this case,

$$
\kappa_{\text {eff }}=\pi \mathrm{R}_{\text {blob }}^{2} / \mathrm{M}_{\text {blob }}
$$

As a particular subset of this family of solutions, Bonnell, Bate \& Zinnecker (1998) considered building up massive stars by coalescence of intermediate-mass stars within a very dense protostellar cluster (see also Bonnell \& Bate 2002, Zinnecker \& Bate 2002). We return to this issue in more detail below.

Opacity modifications owing to coagulation of dust and dust destruction processes during the collapse phase were calculated by Suttner \& Yorke (2001) for three different detailed dust models: compact spherical particles, fractal BPCA (ballistic particle-cluster agglomeration) grains, and fractal BCCA (ballastic cluster-cluster agglomeration) grains. (BPCA dust consists of grains with widely different grains sizes, whereas BCCA dust is formed by coagulation of similar-sized grains). Assuming axial symmetry, Suttner \& Yorke followed the dynamics of gas and 30 individual dust components. They found that even during the early collapse and the first $\sim 10^{4} \mathrm{yr}$ of dynamical disk evolution, the initial dust size distribution is strongly modified. Close to the disk's midplane, coagulation produces dust particles of several tens of $\mu \mathrm{m}$ in size (for compact spherical grains) up to several millimeters in size (for fluffy BCCA grains). In contrast, in the vicinity of the accretion shock front located several density-scale heights above the disk, large velocity differences inhibit coagulation. Dust particles larger than about $1 \mu \mathrm{m}$ segregate from smaller grains behind the accretion shock. Owing to the combined effects of coagulation and grain segregation the IR dust emission is modified. Within the accretion disk, an interstellar medium dust size distribution (Mathis, Rumpl \& Nordsieck 1977) provides a poor description of the general dust properties. Nevertheless, the radiative force acting on material infalling from the envelope to the disk is hardly affected by coagulation (see also Weidenschilling \& Ruzmaikina 1994). 


\subsubsection{Reduce the Effective Luminosity}

Nakano, Hasegawa \& Norman (1995) and Jijina \& Adams (1996) pointed out that, because we expect accretion to proceed through an accretion disk, radiation pressure could blow away the tenuous polar regions but not the massive disk. Yorke \& Bodenheimer (1999) and Yorke \& Sonnhalter (2002) studied this effect quantitatively and substantiated this claim through numerical simulations. They found that, whereas the central object may emit radiation isotropically, the radiation field quickly becomes anisotropic farther from the center. For an outside observer, and in particular, for a dust grain attempting to accrete onto an existing protostellar disk, the radiative flux close to the equatorial plane can be much smaller than the component parallel to the rotation axis. This so-called flashlight effect (beaming of radiation in the polar direction) occurs whenever a circumstellar disk forms.

Yorke \& Bodenheimer (1999) considered the evolution of a $10-\mathrm{M}_{\odot}$ molecular fragment that produced a $8.2-\mathrm{M}_{\odot}$ star (their case $\mathrm{F}$ ). Figure 7 depicts an intermediate stage of the evolution, after $7 \mathrm{M}_{\odot}$ of material have accreted onto the central protostar, while $2.8 \mathrm{M}_{\odot}$ have accumulated in a circumstellar disk and $0.2 \mathrm{M}_{\odot}$ still reside in an larger extended infalling envelope. Inner and outer disk accretion shocks (density and temperature discontinuities) are visible above and below the disk. The dark red regions with an opening angle of about $45^{\circ}$ show preferential heating of polar regions by the protostar. The light red regions within $45^{\circ}$ of the equator beyond $\sim 1000 \mathrm{AU}$ indicate shadowing by the disk.

Yorke \& Bodenheimer (1999) estimated that the edge-on and pole-on bolometric fluxes can differ by more than a factor of 30 after about one-half of the mass of a $2-\mathrm{M}_{\odot}$ collapsing protostellar clump has accreted onto the protostar. The difference in radiative acceleration is much greater than this factor of $\gtrsim 30$, however, because the edge-on flux is dominated by the far-IR, which is far less effective at radiatively accelerating dusty gas than mid- and near-IR light seen pole-on. Yorke \& Sonnhalter (2002) showed by calculating frequency-dependent radiation transfer that the flashlight effect is further enhanced by the fact that the central star's optical and UV radiation blows out material in the polar direction, reducing back-scattering of radiation toward the disk. A similar effect 
has been reported by Krumholz, McKee \& Klein (2005a) from polar cavities blown free by outflows originating close to the star. Thus, photons emitted or scattered into these directions will not hinder accretion of material within the disk or material in the disk's shadow regions.

Although the flashlight effect allows dusty material to come close to the central source via a circumstellar disk, the material to be accreted eventually encounters optical and UV radiation from the central source. For this material to be accreted rather than blown out by radiation, the dust must be largely destroyed or it must have coagulated into larger particles so that the opacity is dominated by the gaseous component.

Even though no massive disk has yet been directly observed around a mainsequence $\mathrm{O}$ star, there is much indirect evidence that such disks exist (see the review by Zhang 2005 and our more detailed discussion in Section 6). A compelling argument that disks exist during the early phases of massive star formation is the observation of massive bipolar outflows. Such massive outflows are probably powered by disk accretion, and, as in their low-mass counterparts, the flow energetics appear to scale with the luminosity of the source (see Shepherd \& Churchwell 1996a,b; Richer et al. 2000; Henning et al. 2000; Wu et al. 2005).

If the primary source of the massive star's material is accretion from the surrounding molecular core, then a circumstellar disk should be the natural consequence of the star-formation process even in the high-mass case. However, it should be difficult to observe disks around massive stars. The high far UV and extreme UV fluxes associated with high-mass stars will begin to photoevaporate the disks on timescales of $\sim 10^{5} \mathrm{yr}$ (Hollenbach, Yorke \& Johnstone 2000). The results will be observable as deeply embedded ultracompact HII-regions with comparable lifetimes (Richling \& Yorke 1997). The fact that disks around O stars photoevaporate so quickly provides negative feedback for disk accretion. This limits the build-up of more massive stellar objects and may even imply an upper mass limit for star formation. 


\subsubsection{Increase Gravity}

For completeness we mention the fact that the gravitational acceleration is enhanced with respect to radiative acceleration when massive stars form within a dense cluster of not-so-brightly-radiating objects. In this scenario, one requires a density-peaked cluster of low-mass objects embedded within a molecular cloud, with $\rho_{\text {objects }} \gg \rho_{\text {gas }}$. The effective gravity near the cluster's center is enhanced relative to an isolated molecular cloud without the cluster and relative to offcenter regions of the molecular cloud (cf. Keto 2002). If this were the only way to form massive stars, isolated massive stars would exist only in very exceptional cases.

\subsection{Stellar and Protostellar Luminosity Evolution}

Because the luminosity is so critical during accretion up to high stellar masses, one must also consider the luminosity evolution of the accreting object. As discussed by Behrend \& Maeder (2001) and Yorke (2002), Figure 8 shows that protostars do not evolve along premain-sequence tracks until they land on and remain at a unique spot on the main sequence where hydrogen burning starts. Rather, they reach the main sequence - that is, the hydrogen-burning state - well before they have finished accreting mass. After that, they continue growing in mass and evolve up the main sequence until they run out of material to accrete. This means that an initially low-mass object that gains mass through accretion evolves substantially differently in the HR diagram than would a nonaccreting premain-sequence star of the same final mass.

Figure 8 shows a number of protostellar evolutionary tracks in the HR diagram, assuming a sequence of conceivable accretion rates increasing by factors of 10, but each constant in time. These accreting tracks were calculated by Yorke (2002) and are qualitatively similar to the more detailed stellar evolution calculations by Norberg \& Maeder (2000), Behrend \& Maeder (2001), and - for the lower accretion rates - Palla \& Stahler (1992). Differences to the two former investigations can be attributed to Yorke's (2002) starting mass, $0.1 \mathrm{M}_{\odot}$ instead of $1 \mathrm{M}_{\odot}$, and the differing accretion rates. In all cases published to date, not only do the tracks of accreting objects consistently lie slightly below the equilibrium 
deuterium-burning birthline, but the qualitative effect of more rapid accretion is to shift the tracks away from the birthline. (In this context, the birthline is defined as the point on the Hayashi track at which equilibrium deuterium burning begins in a nonaccreting star. Palla \& Stahler (1993), however, use the concept of birthline as the evolutionary track of accreting premain-sequence stars in the HR diagram. Deuterium burning of newly accreted material keeps these tracks well above the main sequence until accretion stops.) The tracks of accreting stars eventually converge to the main sequence and follow along the ZAMS as more material is added. For example, at an accretion rate of $10^{-3} \mathrm{M}_{\odot} \mathrm{yr}^{-1}$, hydrogen burning begins at $\mathrm{t} \simeq 1.3 \times 10^{4} \mathrm{yr}$, after $\sim 13 \mathrm{M}_{\odot}$ have been accreted.

The tracks discussed above do not display the degree of bloating seen by Kippenhahn \& Meyer-Hofmeister (1975) for accreting main-sequence stars $\mathrm{M}_{*} \sim 5-$ $10 \mathrm{M}_{\odot}$. Yorke's (2002) simplifying assumption of thermally adjusted (pre)stellar objects is not strictly fulfilled, especially for the $\dot{\mathrm{M}}_{\mathrm{S}-\mathrm{acc}}=10^{-3} \mathrm{M}_{\odot} \mathrm{yr}^{-1}$ case. Norberg \& Maeder (2000) did not consider accretion rates $\dot{\mathrm{M}}_{\mathrm{S}-\mathrm{acc}}>10^{-4} \mathrm{M}_{\odot} \mathrm{yr}^{-1}$ and Behrend \& Maeder (2001) attained high accretion rates $\dot{\mathrm{M}}_{\mathrm{S}-\mathrm{acc}} \gtrsim 10^{-3} \mathrm{M}_{\odot} \mathrm{yr}^{-1}$ for high-mass objects $\mathrm{M}_{*} \gtrsim 25 \mathrm{M}_{\odot}$ only. Palla \& Stahler $(1992,1993)$ found significant bloating for the highest accretion rate $10^{-4} \mathrm{M}_{\odot} \mathrm{yr}^{-1}$ they considered, attributable to shell deuterium burning. We expect that (proto)stars accreting at extremely high rates are not fully thermally adjusted and thus bloat up. The degree of bloating depends on the accretion rate and mass of the accreting object (Kippenhahn \& Meyer-Hofmeister 1975).

We remind the reader that these theoretical tracks in the HR diagram do not reflect the actual observable bolometric luminosities of accreting protostars nor do they reflect the observable effective temperatures. Plotted in the HR diagram are the intrinsic (proto)stellar properties, from which the observed properties must be derived by multidimensional radiative transfer calculations (e.g. Indebetouw et al. 2006). Note that much of the accretion luminosity, $\mathrm{L}_{\mathrm{acc}}$, is indistinguishable from the intrinsic luminosity, $\mathrm{L}_{*}$, of the star. Also, owing to the existence of shock fronts and their postshock relaxation zones, strong deviations from a stellar spectral energy distribution can be expected. The importance of accretion luminosity, $\mathrm{L}_{\mathrm{acc}} \sim \mathrm{GM}_{*} \dot{\mathrm{M}} / \mathrm{R}_{*}$, is seen in Figure 5 . At high accretion rates the $\mathrm{L} / \mathrm{M}$ ratio can attain high values even for low-mass 
cores, so that radiative acceleration can clear out part of the infalling material in the polar regions.

What order of magnitude of mass accretion rate can be expected? In order to produce a star of mass $\mathrm{M}_{*}$ within, say $200,000 \mathrm{yr}$, an average accretion rate of $5 \times 10^{-6} \mathrm{M}_{\odot} \mathrm{yr}^{-1}\left[\mathrm{M}_{*} / \mathrm{M}_{\odot}\right]$ is necessary. Assuming this average accretion rate, we note that during the main accretion phase the luminosity of low-mass stars is dominated by accretion luminosity, whereas for high-mass stars the luminosity is initially determined by accretion but is eventually dominated by the intrinsic stellar luminosity. Of course, the actual accretion rate may vary strongly from this average value. The maximum sub-Eddington accretion rate possible onto a core hydrogen-burning star, assuming electron scattering and the effects of both the intrinsic stellar luminosity and accretion luminosity, can be inferred from Figure 5. Whereas the dotted curve corresponding to an accretion rate of $10^{-3} \mathrm{M}_{\odot} \mathrm{yr}^{-1}$ lies below the value permitted by electron scattering, an accretion rate ten times higher would clearly lie above this value everywhere. Thus, accretion of ionized material onto a stellar core at a rate $\gtrsim 10^{-2} \mathrm{M}_{\odot} \mathrm{yr}^{-1}$ implies super-Eddington accretion.

\subsection{Stellar Evolution Beyond the Zero Age Main Sequence}

As hydrogen burning proceeds, the stars begin to evolve away from the ZAMS even as they accrete material through a disk and lose material through a stellar wind. The star's evolution strongly depends on its mass loss rate and on the internal mixing that is induced by rotation. These effects must be taken into account when modeling these stars and their effect on their environment.

If the stars attain their final mass and become optically visible on a timescale much shorter than their main-sequence lifetime, they will not have evolved far from the ZAMS. If, however, the stars first become optically visible after significant stellar evolution, the traditional concept of the ZAMS as the starting point for newly formed massive stars is flawed. To illustrate this point we compare in Figure 9 the current best estimate of the locations in the HR diagram of spectral types O3 through O9.5 for luminosity classes V, III, and I with theoretical isochrones and evolutionary tracks. It is not surprising that the $\mathrm{O}$ dwarfs 
do not fall on the ZAMS, because we are dealing with average properties over a range of ages. A quantitative explanation of the magnitude and systematic variation of the offset for early to late $\mathrm{O}$ dwarfs, for example a shift of average ages from $\sim 1$ to $5 \mathrm{Myr}$, is still lacking (Martins, Schaerer \& Hillier 2005). Such a shift could occur, because stars of later spectral types have weaker winds and lower UV fluxes and therefore remain embedded in their parental cloud longer.

Table 3: O-stars spectral type versus mass for different luminosity classes (Martins, Schaerer \& Hillier 2005)

\begin{tabular}{cccc}
\hline \hline SpT & \multicolumn{3}{c}{ Luminosity class } \\
\hline & V & III & I \\
\hline B0 & - & - & - \\
O9.5 & 16.5 & 21.0 & 30.4 \\
O9 & 18.0 & 23.1 & 32.0 \\
O8 & 22.0 & 26.9 & 36.8 \\
O7 & 26.5 & 31.2 & 40.9 \\
O6.5 & 29.0 & 33.7 & 43.1 \\
O6 & 31.7 & 36.4 & 45.8 \\
O5 & 37.3 & 41.5 & 50.9 \\
O4 & 46.2 & 48.8 & 58.0 \\
O3 & 58.3 & 58.6 & 66.9 \\
O2.5 & - & - & - \\
\hline \multicolumn{4}{c}{ Note: masses in solar units }
\end{tabular}

We find it useful to end this stellar evolution section with a compilation (Table 3) of the masses of massive stars as a function of spectral type (from O9.5 through O3) and luminosity class (V, III, and I).

\subsection{Ionization Evolution and Cloud Disruption}

Because massive stars are built by accretion, whether from a monolithic collapse or competitively, they will produce an increasing number of hydrogen ionizing photons even while they grow as they become hotter and more luminous. Thus, 
in spite of accretion, hydrogen-ionizing and helium-ionizing fluxes similar to those expected from ZAMS stars are likely (see Figure 10). Indeed, the process of accretion itself is likely to produce hard ionizing radiation in a series of accretion shocks close to the stellar surface. Moreover, powerful winds interacting with surrounding material will produce strong shocks and hard radiation.

Thus, material in the immediate vicinity of the accreting massive star, in particular material in the circumstellar disk and in any nearby star-disk systems, can be ionized. In the case of circumstellar disks, a thin layer of ionized gas on the surface of the disk results. Close to the star, where the escape velocity is much greater than the sound speed, ionized gas remains bound. Ionized gas outside a radius $\mathrm{r}_{\text {evap }}$, that is, the radius where the sound speed exceeds the escape velocity, expands outward. The situation depicted in Figure 11 is likely to result.

The photoionization of circumstellar disks produces an outflowing disk wind of ionized gas that interacts with the stellar wind. Depending on its strength (and the photoionization rate), the stellar wind will be collimated by the ionized disk wind (Richling \& Yorke 1997). The stronger the stellar wind (or the weaker the ionizing photon flux and hence the ionised disk wind), the less collimated the stellar wind will be.

This is different from the case of collimated jets and outflows due to X-winds and disk winds in young low-mass stars, where magnetic fields and forces are involved (Shu et al. 1995). In the high-mass case, although magneto-centrifugal forces cannot be dismissed from influencing the collimation process, we can have rather collimated outflows without magnetic fields. This being said, we suspect that magnetic fields do play a role not only in low-mass but also in high-mass star formation, not least because there are by now at least two cases where strong kilo-Gauss stellar magnetic fields have been inferred from Zeeman spectro-polarimetry, including the oblique magnetic rotator $\theta^{1}$ Ori $\mathrm{C}$ (Stahl et al. 1996, Donati et al. 2002) and the Of?p star HD 191612 (Walborn et al. 2003, Donati et al. 2006). The mismatch in $\theta^{1}$ Ori $\mathrm{C}$ between the magnetic field direction and the stellar spin axis could be an indication that this well-known massive star formed in a collisional process from a merger of two lower mass stars. Finally, we mention the detection of hard X-ray emission from the W3 
massive star-forming region, an indication that embedded massive stars must be magnetically active (Hofner et al. 2002).

Hoare et al. (2007) examined the suggestion first made by Hollenbach et al. (1994) and later calculated numerically by Yorke \& Welz (1996) and Richling \& Yorke (1997) that photoevaporating disks can explain the existence of unresolved ultracompact HII regions (sizes $\sim 0.1 \mathrm{pc}$ ). Many of these have since been resolved with long-baseline radio interferometry and have been reclassifed as hypercompact HII regions (sizes $\sim 0.01$ pc; see Kurtz 2005). The latter most likely represent the individual photoevaporating disks associated with individual deeply embedded O stars (Keto 2007). The phase of photoevaporating disks is the first evolutionary stage after the ionizing flux has turned on. It remains to be seen whether the thermally evaporating flow interacting with a stellar wind can produce the high velocities seen in the broad H92 $\alpha$ recombination line seen toward hypercompact HII regions (Sewilo et al. 2004).

Hoare et al. (2007) concluded that in a later evolutionary phase most ultracompact HII regions need to be interpreted as external photoevaporation of a molecular clump. The longevity of these HII regions is explained by a relative motion of the ionizing source and its stellar wind into the clump, producing a combination of bow shock and unipolar flow (a so-called champagne flow, see the review by Yorke 1986). If this is the case, then the transition from hypercompact to ultracompact HII regions may mark the end of the existence of the accretion disk. The ultracompact HII region phase then marks the beginning of the dissipation of the molecular cloud as the stellar wind and ionizing radiation of the newly formed massive star are able to interact with lower density, more poorly gravitationally bound molecular material.

An important point to make in the evolution of an ultracompact HII region is that the gravitational force of the $\operatorname{star}(\mathrm{s})$ responsible for the HII region should be included (Keto 2002). The gravitational attraction of the star(s) can maintain the accretion flow within the ionized gas and prevent the HII region from expanding hydrodynamically. This is true as long as the radius of the ionization equilibrium is smaller than the radius where the sound speed of the ionized gas, about $10 \mathrm{kms}^{-1}$, approximates the escape velocity (concept of the trapped HII region). Indeed, observations of the H66 $\alpha$ recombination line from 
the ultracompact HII region G10.6-0.4 powered by a compact and very luminous $\left(\sim 10^{6} \mathrm{~L}_{\odot}\right)$ cluster of newly formed massive stars surprisingly show inward motion (Keto 2002). This implies that, despite the high luminosity and ionizing radiation of several $\mathrm{O}$ stars, neither radiation pressure nor thermal pressure has reversed the accretion flow (Keto \& Wood 2006). The ram pressure of the observed accretion flow, with a rate of $\mathrm{M}_{*}=10^{-3} \mathrm{M}_{\odot} \mathrm{yr}^{-1}$, can confine the bubbling HII region and allow the massive stars to continue growing in mass - from ionized gas! One may speculate whether the ionization surrounding the moderately massive stars helps rather than hinders their further growth by accretion (Keto \& Wood 2006), the reason being that ionized gas can couple strongly with any magnetic fields. Magnetic fields can transport angular momentum outward, thereby allowing a high rate of infall to smaller radii to be maintained. Eventually, of course, with the stellar masses steadily increasing, the ionizing flux will increase enough (roughly $\propto \mathrm{M}_{*}^{4}$, see Figure 10) so that the radius of ionization equilibrium grows beyond the critical radius within which the gas is gravitational bound. At this point the HII region will burst free and start to disrupt the dense cloud, preferentially unidirectionally in a champagne flow, revealing the initially deeply embedded cluster (both high-mass and low-mass stars).

\section{Interim Summary}

Despite the obstructing effects of radiation pressure on dust, which would prevent massive stars with masses of more than $30-40 \mathrm{M}_{\odot}$ to be formed in spher-

ically symmetric collapse and accretion models, these and more massive stars can be formed via accretion through a circumstellar disk (i.e., in 2D and 3D models). An accreting star quickly evolves to the main sequence after about $10 \mathrm{M}_{\odot}$ have been accreted, but the star is not yet observable. Still obscured by the material in its vicinity, its appearance will be that of a hypercompact HII region. Radiative acceleration, photoevaporation, and stellar winds eventually destroy the accretion disk, but prior to this, accretion onto the star provides an additional source of luminosity. (Realistically, this accretion is expected to be highly variable and episodic.) A powerful radiation- or magnetically driven outflow in the polar directions and a puffed-up (thick) disk result from the high luminosity of the central source. The details of how disk material ultimately flows onto the star are still unclear - as is often the case for accretion disks. 


\section{MASSIVE STAR FORMATION: COMPET- ING CONCEPTS AND CALCULATIONS}

Three different concepts describing the origin of massive stars have been discussed in the recent literature, each of which may occur in nature, depending on the initial and environmental conditions for the parent molecular clouds (e.g., the Mach number of supersonic turbulence in the clouds and the external pressure in the ISM). These are (1) monolithic collapse and disk accretion, (2) competitive accretion and runaway growth, and (3) stellar collisions and mergers. Whether the parent molecular cloud clumps are magnetically subcritical or supercritical (Shu, Adams \& Lizano 1987, Crutcher \& Troland 2007) may play a crucial role in determining whether massive stars form (1) in isolation or (2) are strongly clustered. Furthermore, in extreme star-forming environments (such as in massive protoglobular cluster clouds), the initial gas densities may have been so high that (3) stellar collisions become an unescapable ingredient in massive star formation. We discuss all these routes toward massive star formation in this section. In addition, we briefly discuss competing, but not mutually exclusive scenarios related to massive star formation stimulated by the pressure of expanding HII regions versus a supernova blast wave. Similarly, we highlight the two competing, but not mutually exclusive processes accounting for runaway OB stars. Finally, the important concept of mass segregation (the fact that massive stars are often concentrated in the centers of star clusters) is reviewed in terms of nature (a birthmark) or nurture (subsequent dynamical evolution).

\subsection{Monolithic Collapse and Disk Accretion}

Yorke \& Sonnhalter (2002) consider the collapse of isolated, rotating, nonmagnetic, massive molecular cores of masses $30 \mathrm{M}_{\odot}, 60 \mathrm{M}_{\odot}$, and $120 \mathrm{M}_{\odot}$ using a frequency-dependent radiation hydrodynamics code. The flashlight effect discussed in Section 3.5 allows material to enter into the central regions through a disk. For massive stars, it is important to take into account the frequencydependent nature of the opacity and the flux within the disk rather than as-

suming either Rosseland or Planck gray opacities. For their $60 \mathrm{M}_{\odot}$ case, Yorke 
\& Sonnhalter find that $33.6 \mathrm{M}_{\odot}$ are accreted in the central regions as opposed to $20.7 \mathrm{M}_{\odot}$ in a comparison gray calculation. Because these simulations cannot spatially resolve the innermost regions of the molecular core, they cannot distinguish between the formation of a dense central cluster, a multiple-star system, or a single massive object. They also cannot exclude significant mass loss from the central object(s) that may interact with the inflow into the central grid cell. With the basic assumption that all material in the innermost grid cell accretes onto a single object, they are only able to provide an upper limit to the mass of stars that could possibly be formed for the cases considered.

Note that the $\sim 43 \mathrm{M}_{\odot}$ star formed during the collapse of the $120 \mathrm{M}_{\odot}$ molecular clump (Yorke \& Sonnhalter 2002) does not represent an upper limit to the stellar mass that is enabled by the flashlight effect. Larger initial masses or a more focused flow along a filament could conceivably lead to even more massive stars. In an ongoing simulation of the collapse of $100-$ and $200-\mathrm{M}_{\odot}$ clouds, Krumholz, Klein \& McKee (2005) report that $\gtrsim 27 \mathrm{M}_{\odot}\left(\gtrsim 25 \mathrm{M}_{\odot}\right.$ for the turbulent case) accreted onto a stellar core. As in the simulations of Yorke \& Sonnhalter (2002), a disk formed around the accreting (proto)stars.

One can speculate on the effect outflows have on the accretion through an accretion disk. The inner part of the accretion disk could well look like the configuration shown in Figure 11. Radiation and the stellar wind from the central star (presumably already hydrogen burning) evacuate a cavity in the polar direction. At the interface between the supersonic outflowing stellar wind and the denser subsonic HII disk atmosphere, some disk material will be removed, but this cannot prevent inward flow of disk material. Inward radial flow of dusty molecular gas is allowed in the equatorial plane of the disk as angular momentum is transfered outward.

In cases of magnetized disks with high radiation densities, photon bubbles can lead to more efficient vertical transport of radiation in the disk (Gammie 1998; Begelman 2001; Blaes \& Socrates 2003; Turner et al. 2005; Turner, Quataert \& Yorke 2007). This can explain super-Eddington accretion in a variety of luminous systems, including accreting compact objects and very massive stars.

Efficient angular momentum transfer could result from weak magnetic fields 
in the disk (Balbus \& Hawley 1991, Hawley \& Balbus 1991, Balbus 2003), from turbulence and/or spiral density waves (Bodenheimer 1995) excited by gravitational instabilities in nonmagnetized (Laughlin \& Bodenheimer 1994) or magnetized (Fromang et al. 2004) disks, or from the tidal effects of nearby stars (Terquem 2001). Indeed, rapid accretion through a disk may be a direct consequence of having nearby companions. This may explain why massive stars are generally members of multiple systems.

Once the disk material crosses $\mathrm{r}_{\text {dust }} \sim 25 \mathrm{AU}\left[\mathrm{M}_{*} / 30 \mathrm{M}_{\odot}\right]^{1.6}$, the radius of dust destruction, its opacity decreases and it is not easily stopped by radiation. It is, however, still unclear how the disk material ultimately flows onto the star. Surely, the disk puffs up close to the star, in analogy to the accretion disks in active galactic nuclei. Beyond $\mathrm{r}_{\text {evap }} \sim 130 \mathrm{AU}\left[\mathrm{M}_{*} / 30 \mathrm{M}_{\odot}\right]$, where the escape velocity is less than $10 \mathrm{~km} \mathrm{~s}^{-1}$, the disk loses material via photoevaporation on a timescale of $\sim 10^{5} \mathrm{yr}$. This is of the same order as the accretion timescale. These competing effects (accretion and photoevaporation) will determine the final mass of the star and perhaps even the upper mass limit.

\subsection{Competitive Accretion and Runaway Growth}

Bonnell et al. (1997, 2001a) present the first 3D numerical simulations of the growth of stellar masses by competitive accretion in small young star clusters. The best way to visualize the idea of competitive accretion is to compare it to an economic model based on two complementary concepts; the real estate concept - "location, location, location" - and the capitalistic concept - "the rich get richer." The former reminds us that environmental influences can be very important, such that being in a fortunate location can significantly promote growth, whereas the latter simply means that the gravitational attraction increases with success - in this case, with the increasing mass of the star. A protostar's ability to grow depends on the size of its accretion domain, i.e., the region from which gas can be gathered. A location in the center of a protostellar cluster is beneficial, as gas flowing down to the center of the cluster increases the gas reservoir available to an individual star. The early birth of a protostar may also give it an unfair advantage in the competition to end up large and 
massive.

Imagine a large, dense molecular gas cloud with a number of protostellar seeds distributed inside the cloud that have initially condensed from some denser portions of the cloud. These condensations or cores subsequently have the chance to grow in mass by accumulation (accretion) of lower density cloud gas from their individual accretion domains (Larson 1978, Zinnecker 1982). These accretion domains are systematically larger for higher mass seeds. With increasing mass, the gravitational spheres of influence keep growing.

Equally important, the amount of material that enters an individual accretion domain depends on the external environment. In isolation, it would simply be proportional to the mean gas density of the region. By contrast, in a larger scale potential such as that provided by a protocluster cloud, the gas density can become significantly larger in the center of the protocluster, as the gas settles into the deepest part of the potential, there to be accreted by the growing protomassive star.

The accretion domain of an off-center protostar is tidally limited by the total mass in the inner part of the cloud, whereas the accretion domain of a protostar in the center of the cloud is the whole cloud. Because the gas reservoir is limited (the cloud has a finite mass), the protostellar masses will eventually compete for cloud gas, especially after the accretion domains start to overlap. The action of the cluster to gather matter from larger distances and focus it toward the accreting stars, combined with the increasing accretion radii of these stars owing to their increasing mass, is what makes competitive accretion such a powerful mechanism.

This is also true in a molecular cloud with hierarchical substructure, in particular for subcluster clumps of gas in a bigger protocluster cloud. The implication is that each subcluster clump is likely to have one most massive protostellar object in its center surrounded by a hierarchy of lower mass objects (Bonnell, Bate \& Vine 2003). When and if those subcluster clumps merge to one big protocluster (see Figure 12), the complex accretion history of each protostar is no longer related to a single local cloud core (Schmeja \& Klessen 2004). In this scenario, the massive stars had accretion histories that were priviledged at every stage - from Jeans-instability protostellar birth to location 
and density in the gas cloud, all factors affecting accretion were more favorable than the average. This is why massive stars are rare. The rarest and most massive of them probably formed in the most favorable conditions by runaway accretion until their gas reservoir was exhausted or dissipated (e.g., by ionization feedback, see von Hoerner 1968 and recently Clarke, Edgar \& Dale 2005).

The above model of competitive accretion has been critized by Krumholz, McKee \& Klein (2005b) on the grounds that Bonnell's SPH simulations start from very strongly gravitationally bound protocluster clouds, while observationally such clouds appear to be supported by turbulent motions. In other words, the simulations use a virial parameter $\alpha=\mathrm{E}_{\text {turb }} / \mathrm{E}_{\text {grav }} \ll 1$ while molecular observations suggest $\alpha \sim 1$. Krumholz, McKee \& Klein (2005b) argue from analytical considerations that protostellar masses cannot grow in such a turbulent medium, not even by a factor of two. The Bondi-Hoyle accretion rate is far too low owing to the high relative velocities between the accreting stars and the turbulent gas. In addition, radiative feedback from the incipient massive star may prevent Bondi-Hoyle accretion altogether (Edgar \& Clarke 2004).

In response to this criticism, Bonnell \& Bate (2006) argue that global initial collapse versus quasi-equilibrium support is not the issue, as their more recent SPH calculations start with initial conditions where the turbulent kinetic energy is close to the gravitational energy. In fact, the scaling laws of supersonic turbulence (Larson 1981) imply small turbulent velocity differences between protostars and their neighboring gas. This then allows for significant growth in stellar mass while more distant high velocity turbulent gas cannot be accreted. In other words, protostars are swept along with their neigboring gas for some time in a similar global motion before they hit more distant and thus lesscorrelated gas. The above Bondi-Hoyle accretion problem is also not as serious as suspected, considering that the most massive star forms in the center of the clump with little motion relative to the surrounding gas.

Future detailed observations of the motions in massive $\left(\sim 1000 \mathrm{M}_{\odot}\right)$ starforming clumps will discriminate between these different dynamical scenarios (small versus large gas motions relative to the protostars, turbulent cloud support, or overall cloud collapse). A first step in this direction was made by Peretto, André \& Belloche (2006) in their dust continuum and molecular study 
of the NGC 2264 clumps, with the conclusion that the observations are most consistent with "a picture of massive star formation intermediate between the scenario of stellar mergers of Bonnell et al. (1998) and the massive turbulent core model of McKee \& Tan (2003), whereby a turbulent, massive ultra-dense core is formed by the gravitational merger of two or more Class 0 protostellar cores at the center of a collapsing protocluster".

\subsection{Stellar Collisions and Mergers}

Historically, the original reason for proposing stellar collisions as a formation process for massive stars was twofold. (a) At the time, radiation pressure on dust was considered a severe hindrance to gas accretion (then assumed to occur spherically symmetrically). Today this concern has gone away (see Section 4.1). (b) The packing of massive stars in dense clusters was too tight, and so there was concern that a sufficiently large gas reservoir for monolithic collapse was not available. This is still a concern, although not all massive stars form in densely packed clusters - many form in widely spread OB associations.

In any case, it is possible that a collisional build-up of high-mass stars can occur, especially for the most massive stars in very dense clusters. The problem is the very high stellar density of already massive or at least intermediate-mass stellar objects that is required to get the process going. We can estimate the threshold stellar number density $\mathrm{n}_{\text {star }}$ or, equivalently, the average star-star

separation $\left(\mathrm{s}=\mathrm{n}_{\text {star }}^{-1 / 3}\right)$ in a dense cluster for stellar collisions (encounters) to be important. Because of the fundamental role of gravitational focusing coupled with a distribution of velocities (i.e., a finite probability of low relative velocities at infinity $\mathrm{v}_{\infty}$ ), the cross section $\sigma_{\text {grav }}$, where

$$
\sigma_{\text {grav }}=\pi \mathrm{R}_{\min }^{2}\left(1+\frac{2 \mathrm{GM}_{*}}{\mathrm{v}_{\infty}^{2} \mathrm{R}_{\min }}\right)
$$

for two stars of mass $M_{*}$ passing each other at periastron within a minimum distance $\mathrm{R}_{\min }$ is vastly enhanced over the geometrical cross section. Gravitational focusing can enhance the effective cross section (by factors of $\sim 10^{4}$ ), thus rendering close stellar encounters in very young stellar clusters realistic. Of course, the condition to meet for massive star growth through mergers is that 
the collision (close encounter) time $t_{\text {coll }}$ for the stars to collide must be shorter than the timescale for stellar evolution of the most massive star in the cluster $(\sim 3 \mathrm{Myr})$.

Using the formula for the stellar collision time per star (Binney \& Tremaine 1987, Dale \& Davies 2006), i.e.,

$$
\tau_{\text {coll }}=\frac{1}{\mathrm{n}_{\mathrm{star}} \sigma_{\text {grav }} \mathrm{v}_{\mathrm{rms}}}=\left[4 \sqrt{\pi} \mathrm{n}_{\text {star }} \mathrm{v}_{\mathrm{rms}}\left(\mathrm{R}_{\mathrm{min}}\right)^{2}\left(1+\frac{2 \mathrm{GM}_{*}}{\mathrm{R}_{\mathrm{min}} \mathrm{v}_{\mathrm{rms}}^{2}}\right)\right]^{-1}
$$

we obtain, when gravitational focusing dominates

$$
\tau_{\text {coll }}=7 \times 10^{7}\left[\frac{\mathrm{n}_{\text {star }}}{10^{6} \mathrm{pc}^{-3}}\right]^{-1}\left[\frac{\mathrm{M}_{*}}{10 \mathrm{M}_{\odot}}\right]^{-1}\left[\frac{\mathrm{R}_{\mathrm{min}}}{1 \mathrm{R}_{\odot}}\right]^{-1} \times\left[\frac{\mathrm{v}_{\mathrm{rms}}}{10 \mathrm{~km} \mathrm{~s}^{-1}}\right] \mathrm{yr} .
$$

Here we have normalized the expression with reasonable numbers for the number density, mass, size, and velocity dispersion of the stellar collision partners, assumed to be of equal mass. We ignore binary stars for the time being. Note that the velocity dispersion is the 1 D-velocity dispersion, which is $2.3 \mathrm{~km} \mathrm{~s}^{-1}$ in the Orion Nebula cluster (van Altena et al. 1988), but is $\sim 5 \mathrm{~km} \mathrm{~s}^{-1}$ in denser and more massive cluster cores such as NGC 3603 and R136, or even larger in young globular clusters such as those in the Antennae (Mengel et al. 2002).

Equation 5 is based on equal mass collision partners; however, recently the formula has been extended to cover nonequal mass encounters (Moeckel \& Bally 2006, 2007), as well as larger cross sections due to circumstellar disks and binary components (Davies et al. 2006). This can decrease the threshold stellar number density for collisions from $10^{7}$ or $10^{8} \mathrm{pc}^{-3}$ down to about $10^{6} \mathrm{pc}^{-3}$ (Bonnell \& Bate 2005).

In Figure 13 we show 3D SPH numerical simulations of the collision process of two pairs of stars, one pair of equal mass and one pair of unequal mass.

\section{Interim Summary}

The primary difference between massive star formation by monolithic collapse versus by competitive accretion is that in monolithic collapse the mass is assumed to be gathered before the star-formation process begins, whereas in competitive accretion the mass is gathered during the star-formation process. If the former is true, we should find massive prestellar cores that live for long times. But how did they form in the first place? Perhaps turbulence could support a slow build-up, but turbulence induces density fluctuations, so why does the 
quasi-static core not fragment into a cluster of low-mass stars? (This is the Dobbs, Bonnell \& Clark 2005 versus Krumholz, Klein \& McKee 2007 debate, see also Krumholz 2006). Magnetic fields may help to stabilize massive cores against subfragmentation.

The strength of competitive accretion is that it provides a physical mechanism to gather the matter. The gravitational potential of the protocluster clump or cluster of stars funnels a significant fraction of gaseous material to the cluster center, there to be accreted by the protomassive stars. In addition, the steep nonlinear mass dependence of the accretion rate onto a gravitating point mass - the Bondi-Hoyle rate - naturally gives a steep, power-law IMF, close to the observed IMF in young clusters (see Bonnell, Larson \& Zinnecker 2007). In this scenario, massive star formation is explained in the context of low-mass star formation (cospatial clusters).

Stellar mergers will be rare and only relevant for the most massive stars in the richest young clusters (such as young globular clusters). One reason to invoke stellar mergers is that ongoing competitive accretion (mass loading) increases the stellar density in the cluster center (mass segregation at birth), potentially to the point where grazing collisions become unavoidable. Stellar collisions with small impact parameters might be the process that forms rapidly rotating massive stars and hence the progenitors of (slow, long-duration) gamma-ray bursts.

\subsection{Triggered OB Star Formation}

The classical model of triggered OB star formation goes back to Elmegreen \& Lada (1977). The idea is that the ionization shock front of one group of massive stars provides the external pressure to compress adjacent molecular cloud layers, thereby inducing the formation of a new group of massive stars, which in turn, by the same process, induces the formation of another generation of massive stars and so on. The Elmegreen \& Lada (1977) model was developed to explain the sequence of spatially distinct OB subgroups in nearby OB associations such

as Orion OB1 or Sco-OB2 (Blaauw 1964, 1991). It is important to note that the Elmegreen \& Lada model did not predict the formation of the observed 
coeval, low-mass T Tauri star population in the subgroups of OB associations (Preibisch \& Zinnecker 2007, Briceño et al. 2007). The low-mass population was assumed to form independently and in many locations spread out over the cloud. Hence the Elmegreen \& Lada (1977) process of sequential triggered star formation, contrary to accepted wisdom, may not be the main mechanism that accounts for the existence of OB subgroups. Rather, supernova triggering could be at work, assuming that a supernova blast wave can trigger both high-mass and low-mass stars at the same time (which would then explain the coevality of the high-mass and low-mass stars). Numerical simulations along these lines are just beginning (e.g., Melioli et al. 2006).

However, there are several clear cases known in our Galaxy where expanding HII regions have swept up molecular gas at their periphery and in which new massive stars have formed or are about to form. The latter is indicated by luminous IR sources (e.g., Sh 104 and RCW 79, Zavagno et al. 2005; RCW 108, Comerón, Schneider \& Russeil 2005). This is indeed reminscent of the Elmegreen \& Lada (1977) model of triggered sequential star formation (the socalled collect and collapse scenario, Elmegreen 1998; see also Whitworth et al. 1994 and Dale, Bonnell \& Whitworth 2007). However, according to Elmegreen \& Lada (1977), it may be expected that in a two-stage starburst (cf. Walborn \& Parker 1992) the second generation protocluster hosts only a small group of high-mass OB stars without the concomitant multitude of low-mass stars. Such a small N-body group, much like the Orion Trapezium system, would then be highly dynamically unstable and could help explain the occurrence of runaway OB field stars (Clarke \& Pringle 1992, Allen, Poveda \& Hernández-Alcántara 2004). An important implication of this scenario would be that the HII region of a big star cluster (e.g., NGC 3603) is not able to trigger the formation of a similarly massive second generation star cluster, but only a smaller mass cluster (e.g., IRS9 in NGC 3603; see Nürnberger 2003), potentially with a top-heavy stellar IMF; similarly, the R136 cluster in 30 Dor does not seem to trigger the formation of a new massive cluster but just a few small groups of embedded protostars (Rubio et al. 1998, Walborn et al. 1999, Brandner et al. 2001, Walborn, Maíz-Apellániz \& Barbá 2002).

A very interesting case is N81, one of the most compact HII regions in the 
Small Magellanic Cloud (SMC). N81 is isolated in an area of low extinction in Shapley's wing, $1.2 \mathrm{kpc}$ away from the main body of the SMC. In contrast to other compact HII regions, typically located within or at the edge of giant HII regions, it appears that SMC N81 has been formed in isolation. The study of its stellar inventory shows that this 'high-excitation blob' is ionized by at least eight near-ZAMS O stars in an instantaneous burst (Heydari-Malayeri et al. 2002, 2003). The question here is, Where is the trigger? How did these massive stars form?

Back to the Galaxy, another region of note is the Carina nebula powered by the most extreme grouping of massive stars in the southern Milky Way (Smith \& Brooks 2007). Here 65 O stars (including many in the clusters Tr14 and Tr16, but excluding $\eta$ Car) provide a total of $\sim 10^{52}$ erg of kinetic energy and a Lyman continuum luminosity of $\sim 10^{51}$ photons $^{-1}$, creating a giant superbubble. Most of the bubble (seen as polycyclic aromatic hydrocarbons in Spitzer/MSX images) resides as atomic gas in the photodissociation regions and not in dense molecular clouds. The synchronized star formation around the periphery of Carina strongly suggests that star formation was triggered by stellar winds. The second-generation population appears to involve a cascade toward preferentially intermediate-mass and low-mass stars (Smith \& Brooks 2007), but the situation may change soon when $\eta$ Car and its siblings explode as supernovae, rejuvenating massive star formation. The idea that it is supernovae rather than HII regions and stellar winds that stimulate wide-spread massive star formation is not new (see Herbst \& Assousa 1977 for the Canis Majoris star formation region, see also Gerola \& Seiden 1978 for galactic spiral arms, and the pioneering paper by Öpik 1953). However, what is new is the evidence from the Upper Scorpius OB subgroup data that supernovae can trigger both high-mass and low-mass star formation in an OB subgroup at the same time (Preibisch \& Zinnecker 2007). Supernovae need a critical distance from a molecular cloud not too close and not too distant - to be an effective trigger for star formation because of momentum transfer of the blast wave onto prestellar cores (Vanhala \& Cameron 1998, Vanhala et al. 1998). Other triggering mechanisms, like radiatively driven implosion of globules, also operate, but seem to be secondary processes, forming only small stellar groups rather than whole OB subgroups 
with thousands of stars (for a review of a whole variety of triggering mechanisms, see Elmegreen 1998).

The Carina star-forming region (diameter 150 pc, age $3 \mathrm{Myr}$, total IR-luminosity $\left.\sim 10^{7} \mathrm{~L}_{\odot}\right)$ may be the galactic analog of giant extragalactic HII regions such as NGC 604 in M33 (size 40 arcsec or 140 pc), a very large OB association (see Maíz-Apellániz, Pérez \& Mas-Hesse 2004).

Closer to home, the Orion Nebula cluster is likely an example of triggered massive star formation. The appearance of the Orion A and B molecular clouds suggest an interaction caused by energy input from the Orion OB1ab association subgroups (Blaauw 1991). Furthermore, the formation of the nearest massive star in the Orion BN/KL region, which hosts a very bright IR source, has probably been triggered by the Orion Trapezium star's HII region pushing against the Orion Molecular Cloud right behind the Trapezium cluster (see Bally 2002).

Recent Spitzer/IRAC Galactic plane observations (Churchwell et al. 2006) have revealed dozens of parsec-sized bubbles, formed by hot young stars in massive star forming regions. Among the 80 or so ring-like structures produced by young OB stars, several show secondary bubbles on the rim of the primary bubbles, suggestive of triggered star formation. However, is this morphological evidence conclusive, i.e., is triggering necessary to explain such spatial correlations? In an interesting paper, Dale, Clark \& Bonnell (2007) attempt to address this and similar issues. They conduct SPH simulations to examine the difference between triggered and revealed star formation. They study the impact of irradiation by an external source of ionizing photons on a turbulent massive molecular cloud and compare the results (the number and type of stars formed) with a control simulation where the turbulent cloud evolves without the impact of the irradiation. They find that, although the external ionization has a dramatic effect on the morphology of the model cloud, its impact in terms of extra star formation is surprisingly minor; the feedback effects can be both positive and negative, accelerating the formation of some objects and delaying the formation of others. Only a few objects form that would otherwise not have formed, and the effect of induced star formation on the overall star formation efficiency is less than a factor of two. 


\subsection{Dynamical Evolution: Mass Segregation and Run- away OB Stars}

\subsubsection{Mass Segregation}

Massive stars are often found near the centers of star clusters but not exclusively. Examples where massive stars are preferentially located in and near the cluster center include the Trapazium in the Orion Nebula cluster (Hillenbrand 1997) or the WR and O stars in the NGC 3603 cluster (Drissen et al. 1995). The issue is whether this is a birthmark (we call this effect prompt mass segregation) or whether this is an N-body evolutionary effect after birth (in which case, we call it dynamical mass segregation). The way to decide between these two possibilities is to estimate the dynamical time for massive stars to sink to the center of the gravitational potential from the half-mass radius of the cluster. If this timescale turns out to be too long, i.e., longer than the age of the cluster, dynamical mass segregation is ruled out and prompt mass segregation is indicated (e.g., Bonnell \& Davies 1998, who infer that mass segregation in the Orion Nebula cluster must have been prompt and hence an important constraint for understanding massive star formation).

\subsubsection{Runaway OB Stars}

Runaway OB stars were defined by Blaauw (1961) as massive stars with radial velocities in excess of $40 \mathrm{~km} \mathrm{~s}^{-1}$. Their high space velocities prompt the question of how they were accelerated to such kinetic energies. The original proposal involved an asymmetric supernova in a massive binary system that provided the kick to eject the companion from the system (Blaauw 1961). A strong prediction of this model is that the runaway massive stars should themselves be single.

More recently, an alternative suggestion for the runaway phenomenon was put forward (Poveda, Ruiz \& Allen 1967, Gies 1987). Here the idea is that massive stars in dense cluster cores (which is their preferential location) undergo dynamical three-body encounters, especially if a massive binary is involved. In such gravitational interactions, potential and kinetic energy are exchanged: The binary orbit shrinks, and the intruder extracts the potential energy and converts 
it into the corresponding kinetic energy. It can also happen that an exchange reaction occurs so that one of the binary components (usually the lighter one, rather than the intruder) is ejected. Conservation of momentum leads to a recoil for each of the two system components, so they fly apart in roughly opposite directions. In this scenario, the runaway stars need not be single but can, in principle, be binaries, at least in $50 \%$ of the cases.

Hoogerwerf, de Bruijne \& de Zeeuw (2001) give evidence that both processes for runaway OB stars occur in nature: while $\zeta$ Ophiuchi is a very high proper motion massive O9.5 star without an antipode in the opposite direction, and hence an example for the supernova scenario, another famous case, that of $\mu$ Columbae and AE Aurigae, argues for the dynamical interaction scenario, where two hard binary systems likely had a close encounter in the $5 \mathrm{Myr}$ old NGC 1980 Orion cluster, in which two of the binary components were set off in opposite directions with speeds of around $100 \mathrm{~km} \mathrm{~s}^{-1}$. In their wake is $\iota$ Ori, a tight, eccentric, massive binary (Gualandris, Portegies Zwart \& Eggleton 2004), known to be classic, colliding-wind X-ray binary. Other famous examples of massive runaway stars include $\lambda$ Cep, $\xi$ Per, and $\alpha$ Cam (astronomy picture of the day on 24 Nov 2006).

The dynamical ejection model for runaway stars would predict not only very fast ejection speeds, but also slower ones, as milder interaction events with wider, i.e., softer, binary pairs can occur (Kroupa 2000). This must be kept in mind when discussing the origin of massive field stars: with an escape speed of only $5-10 \mathrm{~km} \mathrm{~s}^{-1}$, a massive $\mathrm{O}$ star with an age of around $3-5 \mathrm{Myr}$ could still travel $15-50 \mathrm{pc}$ from its cluster birth place. Some 40 nearby field O stars are known and at least half of them can be associated with a cluster origin (de Wit et al. 2004); for the rest the situation is unclear. It is interesting to recall that the spectroscopic and visual binary frequency among these runaway $\mathrm{O}$ stars is very low (Mason et al. 1998). 


\section{MASSIVE STAR FORMATION: BINARY AND MULTIPLE SYSTEMS}

Some of the most important clues toward understanding the formation of massive stars, that have been neglected in previous reviews (e.g., Massey 2003), come from their high frequency of binary and multiple systems, together with an analysis of their properties (period distributions, mass ratios, orbital eccentricities); see Section 2.4 and the discussion later in Section 6.2. Massive stars often come in hierarchical triples with an almost equal-mass close massive binary and a third more distant companion. One example includes $\theta^{2}$ Ori A in Orion's Bar (see Preibisch, Weigelt \& Zinnecker 2001). Another is $\sigma$ Ori in the center of the namesake cluster, whose hierarchical configuration is even more complex (see Sanz-Forcada, Franciosini \& Pallavicini 2004). These close massive binaries likely play an important role in the evolution and age dating of starburst galaxies, as pointed out by van Bever \& Vanbeveren (1998), and an equally important role in estimating supernova rates and the rates of highand low-mass X-ray binaries (see Verbunt 1993; see also H.A. Kobulnicky, C.L. Fryer \& D.C. Kiminki, submitted). Massive close binaries also bias and obfuscate measurements of the velocity dispersion in dense starburst clusters (see Bosch et al. 2001).

It seems worthwhile and appropriate to summarize the various formation processes of massive binary and multiple systems. The following five processes could be relevant.

\subsection{Disk or Filament Fragmentation}

This is the most obvious mechanism; it is similar to suggestions of how to form low-mass T Tauri binaries from filament fragmentation (Zinnecker 1991, Bonnell \& Bastien 1992, Monin et al. 2007). A filamentary geometry may play a key role in the fragmentation process, because the isothermal case is a critical one for the collapse of a cylinder: the collapse and fragmentation of a cylinder can continue freely as long as the temperature continues to decrease, but not if it begins to increase (Larson 2005). As for disk fragmentation, gas 
thermal physics controls the non-linear outcome of gravitational instability in low-mass circumstellar disks (Durisen 2001). The fragmentation of massive disks has recently been studied analytically by Kratter \& Matzner (2006). They found that these disks are unstable to fragment if they are cold enough, and catastrophically so when $\dot{\mathrm{M}}>10^{-3} \mathrm{M}_{\odot} \mathrm{yr}^{-1}$. This mechanism accounts only for initially wide binaries. Cold rotating Keplerian disks produce circular orbits; sub-Keplerian disk rotation or cold filaments tumbling end over end produce highly eccentric orbits. $\theta^{1}$ Ori $\mathrm{C}$ may be an example of the latter (Kraus et al. 2007). Note that fragmentation is different from fission, the splitting up of a hydrostatic, rapidly rotating body. Fission does not work for a compressible fluid (Tohline \& Durisen 2001); it only leads to the ejection of spiral arms and torques that slow down the rapid stellar rotation.

\subsection{Accretion onto a Low-Mass, Wide Binary}

This is a less obvious but potentially key mechanism to form tight massive binaries. Speculated upon by Maeder \& Behrend (2002), it was worked out by Bonnell \& Bate (2005). These authors realized that the orbital separation of the components of close binaries is much smaller than the Jeans length at reasonable gas densities and temperatures. Therefore, they investigated the physical idea that the orbital separation of an initially wide binary would shrink by letting both components accrete and grow in mass; the final separation then depends on the orbital angular momentum of the accreted material, as follows:

Let us consider the angular momentum $\mathrm{A}=\mathrm{Mv} \times \mathrm{R}$ of a binary system. Because of $\mathrm{v} \propto(\mathrm{M} / \mathrm{R})^{1 / 2}(\mathrm{v}$ is the Keplerian orbital speed),

$$
\mathrm{A} \propto \mathrm{M}^{3 / 2} \mathrm{R}^{1 / 2}
$$

where $\mathrm{M}$ is the instantaneous total mass of the binary system and $\mathrm{R}$ is the separation of the binary components. If the accreted material has zero net angular momentum, as would be expected if the infall were spherically symmetric, then $\mathrm{A}$ remains constant $\left(\mathrm{A}=\right.$ const), implying that $\mathrm{R} \propto \mathrm{M}^{-3}$, i.e., the binary separation should be a strong function of the binary mass. If, instead, the accreted material has constant specific angular momentum $(\mathrm{A} / \mathrm{M}=\mathrm{const})$, then the total angular momentum will scale with the mass of the binary $(A \propto M)$, 
implying that $\mathrm{R} \propto \mathrm{M}^{-1}$. Thus it is easy to see that accretion onto a binary system can significantly decrease its separation at the same time that it increases its mass (Bate 2000). In the numerical SPH simulations, the early evolution is well parameterized by the relation $\mathrm{R} \propto \mathrm{M}^{-2}$, intermediate between the above two cases, indicating that the binary is accreting some angular momentum with the mass but that the net specific angular momentum of the accreting gas is decreasing with time. The reason for this is that random velocities of gas at large radii tend to cancel each other out, resulting in accretion of a lower net angular momentum. With an $\mathrm{R} \propto \mathrm{M}^{-2}$ relation, we infer that a low-mass (e.g., $3 \mathrm{M}_{\odot}$ ) wide pair (e.g., separation of order $100 \mathrm{AU}$ ) will become a binary system with a semimajor axis of around $1 \mathrm{AU}$ by the time the system has accreted up to $30 \mathrm{M}_{\odot}$. That is, accretional growth in mass by a factor of 10 will make the separation decrease by a factor of 100 , or more if the growth in mass is more than a factor of ten. This may explain the observed very tight O-star spectroscopic binaries (see Section 2.4).

The component masses, even if unequal in the beginning, would tend to become equal later when higher angular momentum cloud material falls preferentially on the secondary component (Bate \& Bonnell 1997), as the lower mass secondary is, by definition, further away from the center of mass and would thus carry the higher angular momentum in the system initially.

Another very interesting new model to explain the origin of close massive 'twins' is proposed by Krumholz \& Thompson (2006). They invoke mass transfer in close, rapidly accreting protobinaries; this always pushes the initial binaries toward mass ratio unity. Their model is superficially similar to the model of Bonnell \& Bate (2005), but in fact the physical details are quite different, involving the swelling of protostars undergoing deuterium shell burning.

\subsection{Failed Mergers in Stellar Collisions}

If massive stars can form through stellar mergers, then near misses might sometimes form tight and eccentric massive binaries. A necessary condition is that the kinetic energy of the quasi-parabolic encounter can be dissipated as tidal energy (Fabian, Pringle \& Rees 1975) in a gravitationally focused grazing fly- 
by (Zinnecker \& Bate 2002, Dale \& Davies 2006). Apart from the very high stellar number densities required for this process to be significant and frequent, the main problem is the extreme fine tuning of the collision impact parameter. Indeed, the cross section is a very small concentric annulus in impact parameter space that is much smaller than the central area inside the annulus relevant for massive mergers (F. Rasio, private communication).

One way out is disk formation during the tidal disruption of a lower density star by a higher density star in a non-head-on collision; see the example of a grazing collision between a $3 \mathrm{M}_{\odot}$ premain-sequence star and a $10 \mathrm{M}_{\odot}$ mainsequence star in Figure 13. This can lead to subsequent disk-assisted capture of a companion, to be discussed extensively in the next subsection (the socalled 'shred and add' scenario, Davies et al. 2006). A variant of the failed merger scenario is the off-center collision of two protostellar cores with extended accretion envelopes (Stahler, Palla \& Ho 2000). These protostellar envelopes offer a much higher interaction cross section and can provide the necessary orbital drag for collisional massive binary formation (cf. Silk 1978).

\subsection{Disk-Assisted Capture}

If massive stars form via disk accretion, then the large disk radii increase the interaction cross section considerably. This suggests that disk interactions with neighboring stars could assist in capturing binary companions (Bally \& Zinnecker 2005). Although this mechanism has been found insufficient for solar mass stars with disks (Heller 1995, Boffin et al. 1998), recent SPH/N-body simulations by Moeckel \& Bally $(2006,2007)$ convincingly showed that diskassisted capture is much more efficient in a regime suited to massive stars (ca. $20 \mathrm{M}_{\odot}$ ) with large disks (ca. $500 \mathrm{AU}$ ). We note that this process works particularly well in providing massive stars with lower mass companions in rather wide orbits (separation ca. $100 \mathrm{AU}$ ). The same authors also discuss the consequences of a mass-dependent velocity dispersion and of an initial mass segregation for the capture rates. Furthermore, they considered the long-term survival of these binaries in a dense cluster.

The point is that massive binaries with different separations and mass ratios 
form by different processes. Although accretion seems to be the only way to form the observed tight equal-mass binaries in young clusters, fragmentation and disk-assisted capture can form the wider, unequal-mass binaries.

\subsection{N-Body Dynamical Evolution}

Small-N groups of massive and intermediate-mass stars are seen forming in 3D SPH simulations of the collapse and fragmentation of gas-rich protoclusters (e.g., Bonnell, Bate \& Vine 2003). Binary formation in these groups is common and occurs through dynamical three-body capture (different from tidal capture). To begin with, a massive star typically has a lower mass wide companion. With time, an exchange interaction with a more massive third object occurs, thus forming an almost equal mass but still wide binary. The wide binary then shrinks ('hardens') as it takes up most of the binding energy of the small group when the lower mass group members get kicked and escape. The typical final outcome is a tight equal-mass massive binary, with a lower mass wide companion (Bate, Bonnell \& Bromm 2002). This scenario explains many aspects of massive binaries, including the average companion star fraction of 1.5 and the prevalence of short-period spectroscopic systems. Also, the timescales for the N-body dynamics are short enough for the whole process to occur on or before the ZAMS (van Albada 1968, Aarseth 2003).

However, it would seem that the frequency of massive close binaries should correlate with the overall stellar number density in young clusters, which is not what is observed. In fact, at face value the opposite - an anticorrelation - is observed (Mermilliod \& García 2001). We have no real explanation as to how this environmental effect fits into the N-body picture, unless most of the most massive binaries in the densest young clusters have managed to merge into a single object. Stellar mergers are thought to occur in old globular clusters (e.g., Dale \& Davies 2006), and this is one possible explanation for the presence of blue stragglers (younger stars with masses higher than the cluster turnoff mass). Stellar mergers may therefore be even more important in dense, massive, young globular clusters, both now and in the past. 


\subsection{The Origin of Trapezium Systems}

Finally, we address the problem of how Trapezium systems of massive stars, such as the one in the center of the Orion Nebula Cluster $\left(\theta^{1}\right.$ Ori), likely came into being. In brief, the idea is as follows: numerical SPH simulations of supersonic gravo-turbulent fragmentation of a protocluster cloud $\left(1000 \mathrm{M}_{\odot}\right)$ suggest that a collapsing cloud develops a few subclusters (star+gas systems), which subsequently merge into a single cluster entity. Each subcluster carries one most massive star (likely already part of a multiple). Hence the merging of subclusters will result in a central Trapezium-type system (see Figure 14), as observed in the core of the Orion Nebula Cluster (see Figure 15). Note that components A1 and B1 of the Orion Trapezium are spatially unresolved eclipsing spectroscopic binaries; for a summary of the parameters of the Trapezium multiple stars we refer to Preibisch et al. (1999) and Schertl et al. (2003). Note also that component B is itself a Trapezium-like system, indicating the hierarchical nature of massive star formation. The dynamical evolution of Trapezium systems, including stellar ejections, is discussed by Allen, Poveda \& HernándezAlcántara (2004), taking into account their multiplicity substructure. Future studies of Trapezium-type systems will likely concentrate on embedded systems, such as W3-IRS5 (Megeath, Wilson \& Corbin 2005). 


\section{MASSIVE STAR FORMATION: DISCUSSION}

\subsection{Disks and Outflows}

Disks and outflows are a general phenomenon in low-mass star formation that is explained by the accretion-ejection connection (Camenzind 1990, Ferreira \& Pelletier 1995). That is, disk accretion energy powers either a disk wind (Pudritz \& Norman 1983, Pudritz et al. 2007) or an X-wind (Shu et al. 1994, 1995). These in turn are magnetically collimated into a molecular jet. The jet then runs into the parent molecular cloud and local ISM, thereby accelerating entrained ambient molecular gas into a wider momentum-driven molecular flow. Textbook examples include the famous Hubble Space Telescope (HST) source HH30 (Burrows et al. 1996) and the most beautiful IR jets HH211 and HH212 (McCaughrean, Rayner \& Zinnecker 1994; Gueth \& Guilloteau 1999; Zinnecker, McCaughrean \& Rayner 1998; Lee et al. 2006).

Similar but usually less collimated outflows have been observed around more luminous and, hence, more massive young stars (Shepherd \& Churchwell 1996a, b; see the compilation by Beuther \& Shepherd 2005). This has led to the notion that high-mass star formation is a scaled-up version of low-mass star formation. However, as noted by Arce et al. (2007), no highly collimated outflow has been observed for high-mass star-forming regions exceeding $10^{5} \mathrm{~L}_{\odot}$ (corresponding to $\sim 25 \mathrm{M}_{\odot}$ ). Likewise, circumstellar disks have been detected around young B-type massive stars (Zhang 2005), but disks around the progenitors of O-type massive stars in excess of $20 \mathrm{M}_{\odot}$ have eluded detection (Cesaroni et al. 2007). This suggests that simple-minded claims of exact similarity between high-mass star formation and low-mass star formation are partly based on a casual use of the term 'high-mass star' and partly on wishful thinking. Yes, the rotating disks around IRAS 20216+4104 (Cesaroni et al. 1997, 1999; Cesaroni et al. 2005b), G192.16-3.82 (Shepherd, Claussen \& Kurtz 2002), Cep A (Patel et al. 2005), or GL 490 (Schreyer et al. 2006) are wonderful examples of millimeterinterferometric observations. But the central sources are at best early B-type stars (below $20 \mathrm{M}_{\odot}$ ) rather than massive $\mathrm{O}$ stars where radiation pressure and photoevaporation play a role in the formation process. The same seems to be true for the large silhouette disk in M17 (Chini et al. 2004), which is associated 
with a molecular hydrogen jet (Nürnberger et al. 2007).

Let there be no misunderstanding. It is likely that the majority of massive stars forms by disk accretion. But the issue is really whether stars of $50-100 \mathrm{M}_{\odot}$ can also form by some other, more dramatic process like stellar or protostellar collisions (Bonnell, Bate \& Zinnecker 1998; Stahler, Palla \& Ho 2000). One could imagine that the reason why no disks and collimated outflows have been found for the most massive stars in statu nascendi is because their disks are being photoeroded at the same time the stellar mass grows by disk accretion. The combination of a powerful stellar wind and radiative acceleration clear the polar regions of material and magnetic fields, thus leading to poorer collimation for their molecular outflows. Maybe the outflow from AFGL 2591 (Figure 16) is such a case.

The most messy outflow from a massive star-forming region is from the $\mathrm{BN} / \mathrm{KL}$ region in Orion. The OMC1 outflow (mass $10 \mathrm{M}_{\odot}$, velocity $30-100 \mathrm{~km} \mathrm{~s}^{-1}$ ) and the $\mathrm{H}_{2}$ fingers (Allen \& Burton 1993, McCaughrean \& Mac Low 1997, Kaifu et al. 2000) have been interpreted as the result of a powerful explosion that occurred in the center of OMC1 within the last $1000 \mathrm{yr}$. It has been proposed that a dynamical interaction $500 \mathrm{yr}$ ago, possibly leading to a merger, may have produced the OMC1 outflow and $\mathrm{H}_{2}$ finger system (Bally \& Zinnecker 2005). In this scenario, interactions with surrounding gas have decelerated the impulsive outflow powered by the stellar collision by about a factor of two, thereby reconciling the 1000-yr dynamical timescale with the 500-yr timescale for the interaction. The detection of oppositely directed motions in radio sources IRc2-I and BN provides support for models in which the OMC1 outflow was powered by a dynamical interaction between high-mass or intermediate-mass stars in an ultradense environment (Rodríguez et al. 2005a; see also Tan 2004).

Another issue that needs to be appreciated in the context of massive outflows is the question: to what extent do the observed massive outflows trace the combination of stellar wind and intrinsic disk wind? The latter is able to entrain a lot of ambient gas, and care must be taken not to mistake this gas mass for the intrinsic wind mass. This is important because the accretion rate is estimated from the outflow rate, so if the outflow rate is overestimated, the accretion rate is overestimated too. 
Somewhat analogously, one must also be careful when deriving the stellar mass from the luminosity of the source (e.g., using IRAS and submm data) when part of this luminosity is nonstellar and comes from accretion onto the disk and onto the (proto-)star (see Section 3.4.2 and Figure 5). Accretion occurs as long as there is outflow activity, and the highest accretion rate translates into the most violent outflow. Spatially unresolved binary and multiple systems compound the issue (see the next section).

A final issue is the inferred nonlinear mass dependence of the disk accretion rate on the instantaneous central stellar mass (Norberg \& Maeder 2000, Behrend \& Maeder 2001). This is a consequence of a misunderstanding perpetuated in the literature. It dates back to the observed relation that the rate of mass outflow increases roughly linearly with the source luminosity (for a recent plot see $\mathrm{Wu}$ et al. 2005). If the outflow rate scales with the disk accretion rate and the luminosity varies with some power of the stellar mass (e.g., square), then we obtain $\dot{\mathrm{M}}_{*} \sim \mathrm{M}_{*}^{2}$ (Zhang 2005). However, it is a leap of faith to interpret this relation as one of instantaneous quantities rather than one regarding average quantities (the latter simply means that the formation of more massive stars requires higher average accretion rates, whereas the former would imply that the accretion rate keeps increasing with increasing central stellar mass). By completely ignoring the contribution of the accretion luminosity in this scenario, one implicitly assumes that a high luminosity implies a high core mass, as opposed to a high luminosity likely indicating that the accretion rate onto a lower mass (proto)star is high!

A problem with an ever increasing accretion rate is not only how to suddenly stop the accretion, but also the observation that outflow activity is strongest during the earliest phases of star formation rather than during the final phases. In fact, the same problem exists for the turbulent core scenario of massive star formation (McKee \& Tan 2003) where the accretion rate is predicted to increase linearly with time, implying that the most vigorous outflows should occur toward the end of accretion rather than at the beginning. 


\subsection{Binary Statistics and the Most Massive Binary Sys- tems}

The first spectroscopic binary survey among $\mathrm{O}$ stars $(\mathrm{V} \leq 7)$ was conducted by Garmany, Conti \& Massey (1980). They found that 24 out of their 67 O stars are spectroscopic binaries (36\%). Some 15 are double-lined and 4 are single-lined, mostly with 1-10 day periods; the remaining $5 \mathrm{O}$ stars are binary candidates. A later important work is the optical speckle survey of $\mathrm{O}$ stars carried out by Mason et al. (1998). This is a magnitude-limited sample $(\mathrm{V}<8)$ of 227 bright $\mathrm{O}$ stars, observed at 0.1 arcsec spatial resolution (15 new detections). In addition, the previously known spectroscopic binaries were included in a comprehensive analysis. We summarize their results as follows. Besides the 50 known spectroscopic binary systems with periods less than 30 days and typical mass ratios of $0.5-1.0$, there is a similar fraction of wide visual binaries (42 systems with probable orbital motions, with periods larger than $100-1000 \mathrm{yr}$, and typical mass ratios from 0.5 to 0.1 ). Clearly the distribution of mass ratios is different for wide binaries and for close binaries. However there is a huge gap in orbital periods between close and wide binaries. This is a selection effect: Such binaries have been hard to detect with past techniques. Interferometry will be able to find such binaries in the near future. Mason et al. (1998) estimate that the total O star (spectroscopic + visual) binary frequency is about $60 \%$, but they suggest that when the above gap is filled, the frequency may well be $100 \%$. They also note that most binaries occur in clusters and associations and that binaries are less common among field stars and especially among runaway stars.

$10-25 \%$ of all $\mathrm{O}$ stars are runaway stars, whereas among the B stars this fraction is only about $2 \%$ (Gies \& Bolton 1986). The fact that the O-star runaways are 10 times more common than the B-star runaways implies that the secondaries of O-star primaries (which are the ones that get ejected, Leonhard \& Duncan 1990) should also preferentially be O stars. That is, many O-O binaries must have mass ratios skewed to unity (Clarke \& Pringle 1992), in accordance with the observed high frequency of double-lined spectroscopic binaries among O-type stars in young clusters. However, the binary frequency for O-type runaways is generally lower than that in young clusters (Mason et al. 1998). A 
possible explanation could be that the runaway recoil process produces a tight binary and a single massive star ejected in almost opposite directions, thus predicting a spectroscopic binary frequency for runaway $\mathrm{O}$ stars that is half that of young O-star clusters.

Wolf-Rayet (WR) stars are evolved massive stars and, strictly speaking, do not belong to our main-sequence statistics. On the other hand, their progenitors were massive stars that lost much of their mass because of strong stellar winds. The mass loss rate and terminal wind speed depend on metallicity; more metalrich stars lose more mass and lose it more quickly. The initial masses of WR stars cannot easily be determined from observations alone. Many new WR star binaries as well as mixed WR-OB star pairs have recently been discovered. The important question is whether the binary frequency of WR binaries and WROB star pairs depends on metallicity. We leave a discussion of WR-star binary statistics and related topics to Crowther (2007, in this volume).

The O3 stars HD 93129A and HD 93205 in the Carina young star clusters Trumpler 14 and 16 were once thought to be the most massive single stars in the Galaxy (Taresch et al. 1997, Antokhina et al. 2000). In the case of HD 93129A this claim is based on the detailed non-local thermodynamic equilibrium analysis of the UV and hydrogen and helium optical spectrum of this O-type supergiant $\left(\mathrm{T}_{\text {eff }}=52,000 \mathrm{~K}\right)$. This analysis implies a very high bolometric luminosity, $2.5 \times 10^{6} \mathrm{~L}_{\odot}$. The extreme dynamical stellar wind properties $\left(\dot{\mathrm{M}}=-20 \mathrm{M}_{\odot} \mathrm{Myr}^{-1}, \mathrm{v}=3200 \mathrm{~km} \mathrm{~s}^{-1}\right)$ can be used to infer a stellar mass of $130 \pm 20 \mathrm{M}_{\odot}$ (Taresch et al. 1997). However, Walborn (2003) and Nelan et al. (2004), using the Fine Guidance Sensor on HST, recently resolved HD 93129A into two components with a separation of 60 mas or $165 \mathrm{AU}$ and a visual magnitude difference of $0.5 \mathrm{mag}$. This corresponds to component masses of about 80 and $50 \mathrm{M}_{\odot}$. It is unclear how the new stellar parameters can be consistent with the high luminosity and the extreme wind properties, but this example shows strikingly how we can be misled in our conclusions if the binary nature of massive stars is unrecognized.

As for HD 93205, this object consists of an O3V and an O8V noneclipsing pair with an orbital period of about 6 days. Because the inclination angle of the orbit is unknown, we only know that $\mathrm{M}_{1} \cdot \sin ^{3} \mathrm{i}=29 \mathrm{M}_{\odot}$ and $\mathrm{M}_{2} \cdot \sin ^{3} \mathrm{i}=13 \mathrm{M}_{\odot}$ 
(Antokhina et al. 2000). An O8V star should correspond to about $20-25 \mathrm{M}_{\odot}$, so one can tentatively infer a primary mass $\mathrm{M}_{1}=50-60 \mathrm{M}_{\odot}$. Morrell et al. (2001) suggest a spectral type $\mathrm{O} 3.5 \mathrm{~V}$ and a mass of $48 \mathrm{M}_{\odot}$ for the primary of HD 93205, making it one of the earliest main-sequence stars in the Galaxy at this time. There is at least one other massive double-lined spectroscopic binary in the Trumpler 16 cluster ( $\operatorname{Tr} 16-110$ with $\mathrm{P}=3.5$ days), as well as two massive eclipsing binaries ( $\operatorname{Tr} 16-1$ and $\operatorname{Tr} 16-104)$ with $\mathrm{P}=2.2$ days (Rauw, personal communication). Also in the same cluster, there is one of the most massive WR stars in the Galaxy (WR 22, HD 92740), which is the primary of a spectroscopic binary with $\mathrm{P}=80$ days. Its current mass is $45 \mathrm{M}_{\odot}$ (Schweickhardt et al. 1999), corresponding to a ZAMS mass $\sim 80 \mathrm{M}_{\odot}$ (Rauw, personal communication). We refer to Walborn et al. (2002) for further discussion of the most massive stars known (O2V stars). These include Cyg OB2-22A and Pismis 24-1, with masses in excess of $100 \mathrm{M}_{\odot}$ and, up until recently, believed to be single; however, the latter has now been resolved by HST as a visual triple system (see Maíz-Apellániz et al. 2007). A similar case was LBV 1806-20: Eikenberry et al. (2004) determined its mass to be close to $200 \mathrm{M}_{\odot}$, but Figer, Najarro \& Kudritzki (2004) dethroned it, finding that it is most likely a spectroscopic binary. Therefore, at present, the 'Pistol Star' with an estimated initial mass of $200-250 \mathrm{M}_{\odot}$ (Figer et al. 1998) in the Quintuplet Cluster near the Galactic Center is still considered to be the most massive star in the Galaxy.

However, the most massive stars with dynamically measured masses reside in the double-lined spectroscopic, eclipsing binary WR20a: the primary and secondary component masses are $83 \mathrm{M}_{\odot}$ and $82 \mathrm{M}_{\odot}$, respectively, with an error bar of $5 \mathrm{M}_{\odot}$. Rauw et al. (2004) originally discovered the spectroscopic binary nature of the object with an orbital period of about 3.7 days. Bonanos et al. (2004) obtained an eclipse light curve in the I-band and so could determine the inclination angle and hence the masses. Both objects are slightly evolved (SpT WN6ha), and still undergoing core hydrogen burning, but on their way from early O-type to WR stars - with no previous phase of Roche lobe overflow. The binary system lies in the Westerlund 2 cluster associated with the RCW 49 HII region, but interestingly not near the cluster center. Speculation has it that this most heavy close binary system was ejected from the cluster core due to 
a dynamical interaction. A spectacular Spitzer/IRAC image of the RCW 49 HII region and cluster can be found in the Infrared Legacy Gallery at IPAC, courtesy of E. Churchwell and NASA/JPL-Caltech. A glimpse of some other spectacular Spitzer/IRAC images of high-mass star forming regions is given in Brandl et al. (2005), including the embedded proto-OB association W49A.

Additional striking examples of multiplicity among very massive stars can be found in the 30 Dor cluster in the LMC, where Massey, Penny \& Vukovich (2002) have identified four tight, double-lined spectroscopic binaries, three of which are eclipsing systems (R136-38 is the most massive system, with component masses of 57 and $23 \mathrm{M}_{\odot}$, while R136-42 is a close second with component masses of 40 and $33 \mathrm{M}_{\odot}$ (the latter is actually a physical pair of O3V stars with a period of 2.89 days). Other massive stars in 30 Dor, including Melnick 34, are being monitored for radial velocity variations.

Unlike the center of NGC 3603, which is dominated by a massive WR binary system, a recent, still unpublished near-IR integral field spectroscopic survey of the R136 central regions with the ESO-VLT has not identified any WR spectroscopic binaries (T. Moffat \& O. Schnurr, personal communication). This result is contrary to the physical intuition that would lead us to expect the heaviest objects, i.e., massive binaries, to be located in the very center (mass segregation due to dynamical friction).

The center of the R136 cluster that was once considered to host a supermassive star (Feitzinger et al. 1980; Cassinelli, Mathis \& Savage 1981) actually consists of a group of eight massive stars within a projected radius of $0.4 \operatorname{arcsec}$ or equivalently 0.1 pc (Weigelt \& Baier 1985; Pehlemann, Hofmann \& Weigelt 1992), corresponding to a central mass density in massive stars of $10^{5} \mathrm{M}_{\odot}$ per cubic parsec. We do not know how much mass there is in low-mass stars in the center, as two-body dynamical mass segregation could have led to a depletion of low-mass stars there. The density of massive stars alone may not be high enough at this time to enable a runaway stellar collision process, leading to the formation of an intermediate mass black hole, as envisaged by Portegies Zwart \& McMillan (2002). It is enough, though, for the occasional collision between two massive stars, leading to the formation of a rapidly rotating massive object, possibly the progenitor for a gamma-ray burster (Fryer \& Heger 2005, Zinnecker 
2006b). Although the R136 cluster is quite dense in its center, more massive young globular clusters such as those seen in the Antennae merging galaxies may be denser still, and a runaway collision process cannot be excluded.

\subsection{The Universality of the Upper Initial Mass Function}

How can we understand the universality of the upper IMF? Wherever we look, the data seem to be compatible with a Salpeter power-law with a logarithmic slope of -1.35. Even population studies at moderate to high redshift are consistent with the assumption of a Salpeter power law (e.g., Baldry \& Glazebrook 2003).

In essence, two completely different schools of thought have attempted to explain the robustness of the Salpeter IMF despite various kinds of environmental factors that could be expected to change this distribution (such as metallicity, gas pressure, or the density of stellar systems, to name but a few). These are competitive accretion and random sampling of fractal clouds.

As for competitive accretion, this has been discussed extensively in the review of Bonnell, Larson \& Zinnecker (2007) and will not be repeated here. Suffice it to say that competitive accretion of protostars for cluster gas can explain the observed Salpeter stellar mass distribution for massive stars, if most massive stars form in dense clusters (Bonnell et al. 2001b, Klessen 2001a,b, Klessen \& Burkert 2001; for a discussion of the onset and richness of clustering as a function of the most massive cluster star see Testi et al. 1997 and Testi, Palla \& Natta 1999).

If, however, a substantial fraction of massive stars forms in less dense OB associations (Garmany 1994; Clark, Bonnell \& Zinnecker 2005 ) or in isolated places in the field (for example, where was Betelgeuse - a $\sim 20 \mathrm{M}_{\odot}$ red supergiant - born?), then competitive accretion cannot be invoked, and another idea is needed (e.g. Lamers et al. 2002; Li, Klessen \& Mac Low 2003).

Elmegreen (1997) presented such an idea, essentially a geometrical model, with a physical icing on the (geometrical) cake. This model is based on a random sampling of mass in turbulent fractal interstellar clouds (see Figure 17). A star was assumed to get a fixed fraction of the gas mass of the cloud piece in 
which it formed. The mass distribution of the pieces in any hierarchy has a logarithmic slope of -1 (i.e., equal mass in equal logarithmic intervals), so the IMF would seem to end up with this slope, but the sampling rate for pieces was assumed to be proportional to the square root of the local gas density to mimic the dynamical processes that are involved. This local density depends on the level in the hierarchy according to the fractal scaling of density with size (because mass scales with size to a power equal to the fractal dimension, density scales with size to a power equal to the fractal dimension minus three). This density dependence means that lower mass regions are sampled more frequently, and the mass function slope steepens from -1 to -1.35 , which is the Salpeter slope. The net result of this sampling is a mass function for model stars that is indistinguishable from the observed IMF for young clusters and OB associations.

Another interesting attempt to connect star formation to fractal cloud structure was made by Henriksen (1991, see his figure 1), but the details differ from Elmegreen's (1997) model. An important variant of the fractal molecular cloud IMF model is the concept in which the stellar mass function derives directly from the mass distribution of cloud cores. Recent observations show a surprising similarity between the scaled cloud core mass distribution and the stellar IMF (Alves, Lombardi \& Lada 2007).

Yet another alternative for explaining a power-law IMF is due to Basu \& Jones (2004). They note that the power-law tail in the mass function of protostellar condensations and stars arises from the accretion of ambient cloud material on to the condensation, coupled with a nonuniform (exponential) distribution of accretion lifetimes (cf. radioactive decay). Thus, this model assumes that not all condensations accrete for the same time. If we start with protostars with a log-normal mass distribution (possibly a reasonable assumption expected from the central limit theorem, see Zinnecker 1985), this log-normal distribution develops a power-law tail at high masses if the accretion rate is directly proportional to the instantaneous mass of the accreting object and if the probability of stopping accretion is constant in time. The latter implies an exponential probability distribution of accretion timescales with a constant death rate. How general this random accretion model is and how it relates to the competitive accretion model above needs to be further explored (cf. Bate 
\& Bonnell 2005).

Needless to say that the characteristic mass scale of fragmentation and the IMF (a few tenths of a solar mass) is beyond the scope of this review, and we refer to Larson $(1985,2005)$ and also Whitworth, Boffin \& Francis (1998) for an in-depth discussion of this issue. Finally, we refer to Klessen, Spaans \& Jappsen (2007) for the first numerical hydrodynamical calculations of the characteristic stellar mass in starburst regions, predicting a top-heavy IMF. This is naturally explained as a consequence of the elevated thermal Jeans mass in the warmer and dustier starburst environment (including the Galactic Center).

\subsection{The Number of Accreting Massive Protostars in the Galaxy}

In this subsection, we derive an estimate of high-mass accreting stellar objects in the Galaxy (note that we are trying to avoid the term protostars). To this end, we first calculate how much stellar mass is formed in the Galaxy over a timeinterval $t_{\text {acc }}$ corresponding to the accretion phase $(200,000 \mathrm{yr})$. Using a total Galactic star-formation rate (SFR) of $5 \mathrm{M}_{\odot} \mathrm{yr}^{-1}$ (Smith, Mezger \& Biermann 1978, Diehl et al. 2006) and multiplying by $200,000 \mathrm{yr}$, we obtain $10^{6} \mathrm{M}_{\odot}$. Of these, we assume a fraction $\mathrm{f} \approx 0.10$ ends up in massive stars (above $10 \mathrm{M}_{\odot}$, say). The rest goes into intermediate- and low-mass stars according to a Salpeter (1955) field star IMF, with an effective lower mass limit of $0.1 \mathrm{M}_{\odot}$ and upper mass limit of $100 \mathrm{M}_{\odot}$ (see Smith, Mezger \& Biermann 1978, their table 2). A more realistic low-mass field star IMF (Kroupa 2002, Chabrier 2003) would increase the fraction $\mathrm{f}$ by about a factor of 2 , yet would decrease the SFR derived above by about the same factor, thus leaving the product $\mathrm{f} \times \mathrm{SFR}$ approximately constant.

The number $\mathrm{N}_{*}\left(>\mathrm{M}_{*}\right)$ of stellar progenitors in the Galaxy to be found in the accretion phase, which eventually attain a final mass above a given value $\mathrm{M}_{*}$, can then be derived from the two equations (the first solving for $\mathrm{N}_{0}$ ), 


$$
\begin{gathered}
\mathrm{N}_{0} \int_{0.1}^{100} \mathrm{~m}^{-\mathrm{x}} \mathrm{dm}=\mathrm{f} \times \mathrm{SFR} \times \Delta \mathrm{t}_{\mathrm{acc}} \\
\mathrm{N}_{*}\left(>\mathrm{m}_{*}\right)=\mathrm{N}_{0} \int_{\mathrm{m}_{*}}^{\infty} \mathrm{m}^{-\mathrm{x}} \mathrm{dlogm}
\end{gathered}
$$

Here $\mathrm{m}_{*}=\mathrm{M}_{*} / \mathrm{M}_{\odot}$ is the dimensionless stellar mass and $\mathrm{dN} / \operatorname{dlog}(\mathrm{m})=\mathrm{N}_{0} \mathrm{~m}^{-\mathrm{x}}$ is the power-law upper stellar IMF with a slope $\mathrm{x}(\mathrm{x}=1.35$ for a Salpeter IMF). Solving this equation results in the numbers $\mathrm{N}_{*}\left(>\mathrm{M}_{*}\right)$ given in Table 4.

Table 4: Expected number $\mathrm{N}$ of stellar progenitors in the Galaxy to be found in the accretion phase ${ }^{a}$, which eventually attain a final mass above $\mathrm{M}_{*}$, for various logarithmic slopes $\mathrm{x}$ of the $\mathrm{IMF}^{b}$

\begin{tabular}{cccc}
\hline \hline $\mathbf{M}_{*}$ & $\mathbf{N}_{*}$ & $\mathbf{N}_{*}$ & $\mathbf{N}_{*}$ \\
{$\left[\mathrm{M}_{\odot}\right]$} & $\mathrm{x}=1.3$ & $\mathrm{x}=1.35$ & $\mathrm{x}=1.7$ \\
\hline$>10$ & 6300 & 5400 & 1600 \\
$>20$ & 2400 & 2000 & 480 \\
$>30$ & 1300 & 1000 & 220 \\
$>50$ & 480 & 390 & 74 \\
\hline
\end{tabular}

${ }^{a}$ Assumed to last 200,000 yr.

${ }^{b}$ Assuming a total Galactic star formation rate of $5 \mathrm{M}_{\odot} \mathrm{yr}^{-1}$.

The table shows that we can expect about 1000 accreting massive stars in the Galaxy with masses in excess of $30 \mathrm{M}_{\odot}$ (i.e., spectral types earlier than $\mathrm{O} 6.5 \mathrm{~V}$, see Table 3). Assuming $10 \mathrm{kpc}$ as the reference radius of the Milky Way, we see that the average surface density of accreting early O-type massive stars is about 3 objects $/ \mathrm{kpc}^{2}$. This demonstrates the rareness of massive protostars, but is in agreement with the few luminous and totally embedded objects known, such as BN/KL and Cep-A (see Table 5 in the Appendix which provides a list of massive star forming regions within $1 \mathrm{kpc}$ from the Sun and some properties of the exciting stars). 


\subsection{Is There a Maximum Stellar Mass Set by Star Forma- tion?}

In Section 2.5 we reviewed the observational evidence for an upper stellar mass limit and concluded there was such a limiting mass somewhere in the range between 100 and $200 \mathrm{M}_{\odot}$, at least in Population I systems. Here we present a few thoughts on the possible physics of the stellar upper mass limit.

The first question is whether this limit is set by stellar stability considerations or star-formation theory. Ledoux (1941), and later Schwarzschild \& Härm (1959), concluded from linear stability analysis of radial stellar pulsations that there is an upper mass limit of the order of $100 \mathrm{M}_{\odot}$, corresponding to the existence of a vibrational instability owing to nuclear reactions. This refers to massive stars of Population I chemical composition, whereas for lower chemical abundances the unstable mass is higher. Appenzeller (1970) and Ziebarth (1970) concluded, from nonlinear calculations, that this vibrational instability (also known as $\epsilon$-instability) does not have a global disrupting effect, except perhaps in stars with masses greatly exceeding the critical mass. A more recent discussion can be found in Stothers \& Chin (1993). These authors concluded that, for a metallicity equal to or greater than 0.02 , no nuclear-induced pulsational instability developed for masses up to at least $150 \mathrm{M}_{\odot}$. For a metallicity equal to 0.004, corresponding to the value of the Small Magellanic Cloud, the critical mass for such an instability was still very high, around $140 \mathrm{M}_{\odot}$.

Larson \& Starrfield (1971) were the first to stress a possible upper mass limit resulting from star-formation theory. They came up with a value of $50-100 \mathrm{M}_{\odot}$ as a larger and larger fraction of the growing mass is thrown out by radiation before the star reaches stellar conditions.

Today, we realize that the opacity of dusty gas does not determine the upper mass limit, whereas the opacity of ionized gas (electron scattering and UV line blanketing) certainly may. The constraint imposed by dust opacity (see Figure 6) shows that a luminous object with an $\mathrm{L} / \mathrm{M}$ ratio consistent with a main-sequence star $\mathrm{M}_{*}>100 \mathrm{M}_{\odot}$ can still accrete material as long as the object appears to be cool $(<50 \mathrm{~K})$. This allows material to fall onto the disk. Within the disk much of the radial radiation flux is diverted into the polar 
direction (flashlight effect). Provided angular momentum transfer is adequate, dusty material can thus flow radially inward until at some point the dust is destroyed (sublimation radius). Dust-free neutral gas has a lower opacity and it can continue to flow inward. Eventually, as the disk gas gets closer to the accreting star, the gas is ionized and the opacity increases again sharply. Disk models currently do not allow an accurate estimate of the opacity of the ionized disk gas close to the star; the lower limit to the opacity provided by electron scattering limits the maximum L/M to $\sim 6 \times 10^{4}$, well in excess of that expected from a main-sequence star of $\mathrm{M}_{*}=200 \mathrm{M}_{\odot}$.

Because very massive stars also possess strong radiation-driven winds, it is clear that the opacity of ionized stellar gas is sufficiently high that radiative acceleration exceeds surface gravity. The upper mass limit could thus result from the fact that mass loss from the star matches or exceeds accretion. As the linedriven wind mass loss from massive stars is metallicity-dependent (Kudritzki 2002) the upper mass limit would be metallicity-dependent, too. However, if mass loss is by a continuum-driven instability, as suggested for $\eta$ Car and other Luminous Blue Variables (Smith \& Owocki 2006), the upper mass limit would be independent of metallicity.

It is unclear if stellar collisions and mergers in dense stellar systems can beat the opacity-limited accretion discussed above and form stars with masses beyond $200 \mathrm{M}_{\odot}$. The merging of a binary star with two $100-\mathrm{M}_{\odot}$ components is conceivable (Zinnecker 1986, Bonnell \& Bate 2005), doubling the single star upper mass limit. It is likely that a coalescence process would deplete the number density of stars to collide with near the center of a deep cluster potential, thus imposing a density-dependent limiting mass, especially for young clusters undergoing core collapse (Portegies Zwart et al. 2006). Interestingly, in this case, too, the upper mass limit would be independent of the heavy element abundance. We refer to Omukai \& Palla (2003) for a theoretical prediction of the increased upper mass limit $\left(\sim 600 \mathrm{M}_{\odot}\right)$ in zero-metallicity, Population III conditions, albeit based on 1D models. 


\subsection{Evolutionary Sequence}

We can characterize the formation of massive stars by the following crude fourstage evolutionary sequence: $\mathrm{CDMC} \longrightarrow \mathrm{HDMC} \longrightarrow$ DAMS $\longrightarrow$ FIMS. Here CDMC means cold dense massive core, HDMC means hot dense massive core, DAMS stands for disk-accreting main-sequence star, and FIMS denotes the final main-sequence star. The FIMS star is more evolved than the theoretical concept of the ZAMS star, which assumes no prior hydrogen consumption. Of course, the sequence needs a more detailed description. The CDMCs are starless turbulent gravitationally bound condensations; they have near-virial equilibrium and are either on the verge of collapsing or, indeed, already collapsing. In the center an intermediate-mass protostar forms that can heat up the dense massive core, turning it into a HDMC. At this stage, collimated jets and outflows first appear, traced by $\mathrm{H}_{2} \mathrm{O}$ and later by methanol maser emission. The central star grows in mass primarily by disk accretion and quickly becomes a DAMS object powered more by hydrogen burning than by disk accretion. The collimated outflows become less collimated with widening opening angles. At this stage, the accretion disk starts to get photoionized and partly photoevaporated, giving rise to a gravitationally confined hypercompact HII region (HCHII) with broad hydrogen recombination lines.

Eventually the star has accreted its final mass and most of the disk has been dissipated. At this point the ionizing radiation is no longer quenched by the accretion flow and can expand freely into the vicinity of the star, blending together with ionized bubbles from other similarly massive stars. This is when we speak of an ultracompact HII region (UCHII), often formed by a group of ionizing stars within a volume of $(0.1 \mathrm{pc})^{3}$ (e.g., W3-IRS5). UCHII regions frequently exhibit $\mathrm{OH}$ maser 18-cm radio emission [e.g., W3 $(\mathrm{OH})]$, which can be used to obtain a magnetic field strength by measuring the Zeeman splitting of the $1667 \mathrm{MHz}$ line. Subsequently, the UCHII region keeps expanding and evolves into a compact radio HII region and finally into a normal diffuse optical HII region like the Orion Nebula.

How this simple-minded picture changes when we deal with a massive starburst protocluster, like the progenitor of R136 with dozens of massive stars 
born in a small volume, is anybody's guess. It is conceivable that competitive accretion takes place in a gravitationally bound dense HII region. It is even conceivable that competitive accretion turns into cooperative accretion (E. Keto, private communication) which means the ionized flow pulled in by the collective gravity of the cluster is redirected onto the less massive members of the cluster. Instead of being greedy and competing for additional mass with their siblings, the near-Eddington-limit massive stars start to deflect the inflow onto neighboring stars. By doing so, they aid the further growth of their lower mass neighbors. Thus, accretion becomes cooperative! Another possibility is the hierarchical merging of subclusters which naturally leads to prompt initial mass segregation in the resulting final cluster (McMillan, Vesperini \& Portegies Zwart 2007). In this scenario, many but not all subclusters (gas+stars) merge with each other and sink to the center of gravity owing to the loss of kinetic energy in these highly inelastic collisions. There they form a dense stellar cluster of massive stars, which will expel the residual cluster gas. Some subclusters miss and won't collide; these will form massive stars in the cluster outskirts. Such a core-halo structure is observed in many young clusters, including R136 (e.g., Moffat, Drissen \& Shara 1994). Super OB associations like NGC 604 in M33 have more widely spread subclusters that are unable to merge, thus avoiding dense cluster formation (cf. Hunter 1995). 


\section{WHY HIGH-MASS STAR FORMATION IS NOT A SCALED-UP VERSION OF LOW- MASS STAR FORMATION}

In this section, we try to convince the reader that high-mass star formation is indeed different from low-mass star formation, and not merely a scaled-up version of star formation by disk accretion for higher accretion rates. Many complex new physical processes enter the scene for high-mass stars.

To start with, radiative forces on gas and dust play little or no role in the build-up of low-mass, solar-type stars, whereas a substantial fraction of the luminosity of high-mass stars is emitted in ionizing radiation, which introduces new effects such as the photoevaporation of the star's accretion disk and protostellar envelope. This dramatically limits late accretion and the final stellar mass. In addition, the ionizing photons can photoevaporate the disks of the neighboring lower mass stars (cf. the proplyds in the Orion Nebula). The nonionizing far-UV radiation will influence the massive stars' molecular cloud environment by dissociating $\mathrm{H}_{2}$ and $\mathrm{CO}$ molecules. This requires photons of about $11.2 \mathrm{eV}$; even early-type $\mathrm{B}$ stars can produce these photons, but low-mass and intermediate-mass stars cannot. The radiative acceleration of dusty and gaseous matter also leads to radiation-driven bipolar winds, and ionizing radiation can escape through these wind-blown cavities (flashlight effect); however, the bipolar outflows from low-mass stars are generated by magneto-centrifugal forces.

The second big difference between the formation of low-mass and high-mass stars is the fact that massive stars are practically born on the main sequence, whereas low-mass stars spend a considerable part of their youth as contracting premain-sequence objects (30 Myr for a solar-mass star). A massive star, forming by accretional growth from an initially low-mass star with an accretion rate of $10^{-4} \mathrm{M}_{\odot} \mathrm{yr}^{-1}$ begins central hydrogen burning after about $9 \mathrm{M}_{\odot}$ have accumulated $-13 \mathrm{M}_{\odot}$ for an accretion rate of $10^{-3} \mathrm{M}_{\odot} \mathrm{yr}^{-1}$. For low-mass stars, circumstellar disk evolution proceeds during the whole extended premainsequence phase, whereas for massive stars the disk lifetime is very short (less 
than 1 Myr). When we see massive O stars close to the ZAMS (Walborn 2007), their disks have been dissipated (but see Kastner et al. 2006). It is only in the embedded phase that circumstellar CO-bandhead emission and hydrogen emission lines are detected (Bik et al. 2005, Blum 2005), indicating a dense neutral remnant disk with an ionized upper layer. Bipolar outflows are another strong indication of the existence of disks.

A further significant difference between low- and high-mass star formation is the role of competitive accretion in protoclusters. Competitive accretion is far more important for high-mass stars than for low-mass stars. The latter can form directly by Jeans-type gravitational instability and turbulence-induced cloud fragmentation. The former must accrete large amounts of protocluster gas.

Gravitational dynamics (N-body interactions) also have a much greater effect when massive stars are involved. This can best be seen when considering the dynamical ejection of members of massive multiple systems producing runaway OB stars. This phenomenon is largely absent for low-mass stars. It appears that massive stars, when they form in their own local subclusters, are always accompanied by a small group of lower mass stars, some of which stay bound to their big parent star even after subclusters merge. Thus the higher companion star fraction observed for massive stars compared to solar-type stars testifies to a more dynamic scenario for the heavy objects thanks to their higher than average gravitational attraction.

Finally, massive stars have a much bigger influence on triggering new star formation in adjacent regions than low-mass stars. Massive stars provide external pressure in the form of expanding HII regions, stellar winds, and supernovae explosions. They are capable of sustaining sequential and self-propagating star formation (Gerola \& Seiden 1978), a process that low-mass stars are incapable of. Through runaway OB stars, massive star formation can trigger further massive star formation over large (kpc) distances, an important feature in sustaining large-scale nuclear starbursts. We speculate that, if massive star formation is massively triggered, the individual collapse of massive cores is outside-in, instead of inside-out (Banerjee, Pudritz \& Anderson 2006), and always magnetically supercritical (Shu, Adams \& Lizano 1987). In other words, magnetic fields likely play a more passive role in massive star formation, whereas in low-mass stars 
this is opposite.

In defense of the notion of a scaled-up formation picture, some arguments have been raised. Foremost is the observation that collimated outflows occur both in high-mass and low-mass young stars (Beuther et al. 2002, Davis et al. 2004). However, a closer look reveals that collimated outflows and jets do not occur in the most luminous sources, but only in sources with total luminosities up to $10^{4} \mathrm{~L}_{\odot}$ (Shepherd 2005), with one or two exceptions (Garay et al. 2003, Rodríguez et al. 2005b). This implies that massive star formation can be a scaled-up version of low-mass star formation, but only up to early B stars. The outflow morphology does not scale further for O stars, which generate powerful, wide-angle, ionized winds, calling into question the relationship between outflow and accretion (Shepherd 2005).

Another point that has been made relates to the IMF. It has been suggested (see the discussion in Zinnecker 2004) that there should be a feature (knee) in the IMF, at a critical mass where the intermediate-mass and high-mass starformation processes diverge. However, this feature has not been seen and the upper IMF is a power-law with a constant slope (Kroupa 2002). It is unclear how to refute this argument, but the constant slope of the upper IMF may simply imply that stellar collisions and mergers are not a dominant factor in massive star formation, except for the most massive stars (Bonnell \& Bate 2002) where statistical fluctuations can hide a slope change. Collisional growth of massive stars (Bonnell, Bate \& Zinnecker 1998; Bally \& Zinnecker 2005) also seems to be ruled out by recent observations of stellar rotation: There is a continuous behavior of the specific stellar spin angular momentum over the full range of stellar masses, i.e., $\mathrm{J} / \mathrm{M} \sim \mathrm{M}^{0.3}$ between $0.2 \mathrm{M}_{\odot}$ and $50 \mathrm{M}_{\odot}$, indicative of a single stellar formation and angular momentum regulation mechanism (Wolff et al. 2006). However, a collisional process and stellar mergers are not ruled out for the most massive early O-type stars $\left(\mathrm{M}>50 \mathrm{M}_{\odot}\right)$. 


\section{OUTLOOK: RELEVANT FUTURE OBSER- VATIONS}

Here we suggest a number of future observations that would help promote?advance our understanding of massive star formation. The list, however, is by no means complete.

1. IR-observations with an Extremely Large Telescope (ELT)

(diameter $30-42 \mathrm{~m}$ )

As described in Zinnecker (2006a), such a powerful telescope could penetrate the dust extinction of ultracompact HII regions $\left(A_{V}=100-200 \mathrm{mag}\right)$ in the near-IR $\left(\mathrm{A}_{\mathrm{K}}=10-20\right)$ and see the stellar photospheres of massive stars, resolving very tight embedded clusters, such as W3-IRS5 (cf. Megeath, Wilson \& Corbin 2005). There is hope that an ELT might have sharp enough vision to test the prediction (Zinnecker 2006b) that gammaray bursts occur in the centers of young massive protoglobular clusters.

2. Submillimeter observations with the Atacama Large Millimeter Array The spatial resolution of the Atacama Large Millimeter Array (ALMA) at 350 microns ( $\sim 0.01$ arcsec, i.e., $50 \mathrm{AU}$ at $5 \mathrm{kpc}$ ) would allow us to map dense molecular clumps, where massive stars are believed to form, in the dust continuum and see whether fragmentation is occurring (Dobbs, Bonnell \& Clark 2005) or not (Krumholz 2006). ALMA should also be able to measure rotation curves of circumstellar disk gas around massive stars and thus measure their enclosed stellar mass (M. Krumholz, R.I. Klein \& C.F. McKee, submitted).

3. Mid-infrared observations with the James Webb Space Telescope The James Webb Space Telescope will have unparalleled background-limited sensitivity to reveal very embedded populations around young massive stars in the making in near- and far-galactic star forming regions as well as regions like 30 Dor/R136 in the Magellanic Clouds. Progress may also be expected for understanding star formation in the Galactic Center Regions, including resolution of the Arches and Quintuplet clusters and the 
OB populations near Sgr A* (Figer 2003, Kim et al. 2006, Paumard et al. 2006, Martins et al. 2006).

4. Proper motion observations with Gaia

Precise proper motion data of young clusters and OB associations are eagerly awaited, surpassing the limited milli-arcsec precision of Hipparcos (de Zeeuw et al. 1999). This will be crucial to understanding the dynamical nature of $\mathrm{OB}$ associations, such as the question of whether an OB association is just an expanding, dissolving massive star cluster or something of its own (Blaauw 1983, Briceño et al. 2007). Hence Gaia will tell us if the stars of an association expand from a common center or from several centers or from no center at all. Gaia will also provide us with a complete sample of field $\mathrm{O}$ stars and their parallaxes within a few kiloparsecs.

5. Far-IR observations with Herschel

The immediate future will see the launch of ESA's Herschel space observatory, whose Photodetector Array Camera \& Spectrometer (PACS) instrument will make it possible to follow up on Spitzer/MIPS observations and to detect highly embedded massive protostellar sources down to a spatial resolution of 3 arcsec at 60 microns. Surveys with the Spectral and Photometric Imaging Receiver (SPIRE) will allow us to identify and characterize pre-collapse cores at an early evolutionary stage (Boss \& Yorke 1995). PACS together with SPIRE can be used to better constrain the 'collect and collapse' model of triggered massive star formation (Zavagno et al. 2005), discriminating between true triggering versus the compression of pre-existing clumps.

6. Interferometric near-IR observations with VLTI and LBTI

Very high angular resolution observations with closure phases will give us interferometric images (and not just visibilities) of ultracompact HII regions and the stellar content of hot molecular cores. This will allow us to obtain a clearer picture of the maternities of massive stars (Garay 2005), including images of disks and jets as well as astrometric information, such 
as the stellar velocity dispersion, dynamics of the stars in protocluster centers, and the orbital motion of short-period embedded binary and multiple systems.

7. Long-term variability surveys The formation and evolution of massive stars is likely to involve a variety of cataclysmic or eruptive phenomena on short timescales (days to years), such as episodic FU Orionis-like accretion (Chini, private communication) and stellar mergers (Bally \& Zinnecker 2005), or $\eta$ Car-like LBV eruptions (Smith \& Owocki 2006) and even infrared supernovae in luminous starburst galaxies (Maiolino et al. 2002). Without continuous monitoring, these (embedded) events may go unnoticed. This calls for long-term variability compaigns, in the nearinfrared, for example from a medium-sized survey telescope at Dome $\mathrm{C}$ in Antarctica.

8. "Observations" with a Numerical Telescope

As numerical simulations of the star formation process become more refined in $3 \mathrm{D}$ resolution and the microphysics involved (magnetic fields, chemistry, ionization, heating, cooling, dust physics, etc.) the ability to accurately "observe" the evolution at various stages with respect to dust continuum features, line intensities and line profiles increases in importance. In other words, theoretical modeling will have to keep pace with the rapid development of observing capabilities as described above. 


\section{FINAL SUMMARY}

Massive star formation is not a simple scaled-up version of low-mass star formation, particularly when it comes to ZAMS O stars $\left(>20 \mathrm{M}_{\odot}\right)$. The formation of early-type B stars, however, may still be considered a continuation of lowmass star formation. In terms of formation processes, the monolithic collapse and disk accretion model and the competitive accretion scenario are the two opposite ends of a continuum of cases in the accretion theory of O-type massive star formation. The coalescence process, introduced to circumvent the obstacle of radiation pressure, is no longer generally necessary but may still occur in exceptional circumstances, especially for very massive stars in the centers of very dense protoclusters or subclusters with strong initial mass segregation. The observed multiplicity and clustering of massive stars suggests complex Nbody and gas dynamical interactions (tidal, drag, and capture effects) among the youngest stars or protostars that would contribute to the argument against a monolithic collapse of isolated massive protostellar cores.

Massive stars are rare and correspond to the tail of a power-law stellar mass distribution. For a Salpeter IMF, the number of all newborn ZAMS OB stars $\left(>8 \mathrm{M}_{\odot}\right)$ is a mere $10 \%$ of all stars in the mass range of $1-2 \mathrm{M}_{\odot}$. At present, competitive accretion (i.e., runaway growth of a few objects from cluster gas) provides the best physical explanation of the high-mass IMF and its surprisingly universal Salpeter slope. The maximum stellar mass (around $150 \mathrm{M}_{\odot}$ ), long believed to be a result of stellar instability (Eddington-Ledoux limit), might be due to the negative feedback caused by the increasingly destructive radiative erosion of massive accretion disks at increasingly higher stellar masses. This self-limiting star formation can, however, be beaten by stellar collisions under very special circumstances.

Rapid external shock compression (i.e., supersonic gas motions) generating high column densities in less than a local free-fall time rather than slow quasistatic build-up of massive cores may be the recipe to set up the initial conditions for local and global bursts of massive star formation. Such bursts can both enhance or quench further massive star formation (positive and negative feedback), depending on environment (gas density and pressure), but such a discussion is 
beyond the scope of this review. The question of how feedback from massive stars can influence proto galaxy evolution and morphological types (cf. Sandage 1986, Elmegreen 1999, Silk 1997, 2005) should be the topic of a future Annual Reviews article. 


\section{APPENDIX - NEARBY ( $<1 \mathrm{kpc})$ MASSIVE STAR-FORMATION REGIONS}

In this Appendix, we attempt to give a complete table of star formation regions within $1 \mathrm{kpc}$ that contain at least one massive star (see Table 5). Some of these nearby regions of massive star formation are described in more detail by Bally et al. (2005). Moreover, the massive stellar content of nearby (distance $<650 \mathrm{pc}$ ) OB associations are disscussed in the classic Hipparcos paper by de Zeeuw et al. (1999), including Sco OB2, Vel OB2, Per OB2, and Cep OB2 (see their figures 1 and 29). We also should mention the Galactic O-star catalog of Maíz-Apellániz, Pérez \& Mas-Hesse (2004), which includes spectral classification, optical-NIR photometry, multiplicity, and astrometric information, as well as cluster?association membership. (See also the living O-star Web-catalogue http://www-int.stsci.edu/ jmaiz/GOS/GOSmain.html). O stars in open cluster regions can also be selected from the combined photometric and astrometric membership analysis of 520 previously known (Kharchenko et al. 2004) and 130 newly detected (Kharchenko et al. 2005) open clusters. As a final highlight, we show here Westerlund 1, the most massive young star cluster in the Galaxy (Figure 18). 
Table 5: Star formation regions (within $1 \mathrm{kpc}$ ) with massive stars (B2 and earlier)

\begin{tabular}{|c|c|c|c|c|c|}
\hline Region & Sources & Distance & Age & $\mathrm{L} / \mathrm{SpT}$ & References \\
\hline \multirow[t]{2}{*}{ OMC-1 } & $\mathrm{BN} / \mathrm{KL}$ & $450 \mathrm{pc}$ & $\mathrm{emb}^{a}$ & $10^{5} \mathrm{~L}_{\odot}$ & Menten \& Reid 1995 \\
\hline & & & & & Greenhill et al. 2004 \\
\hline \multirow[t]{2}{*}{ OMC-1S } & FIR4 & $450 \mathrm{pc}$ & $\mathrm{emb}$ & $10^{4} \mathrm{~L}_{\odot}$ & Schmid-Burgk et al. 1990 \\
\hline & & & & & Smith et al. 2004 \\
\hline \multirow[t]{2}{*}{ NGC1977 } & HD 37018 & $450 \mathrm{pc}$ & $5 \mathrm{Myr}$ & $\mathrm{B} 1 \mathrm{~V}$ & Makinen et al. 1985 \\
\hline & & & & & Howe et al. 1991 \\
\hline \multirow[t]{2}{*}{ NGC1980 } & $\iota$ Ori & $550 \mathrm{pc}$ & $5 \mathrm{Myr}$ & O9III & Johnstone \& Bally 2006 \\
\hline & & & & & Piskunov et al. 2006 \\
\hline \multirow[t]{2}{*}{ NGC2023 } & HD 37903 & $475 \mathrm{pc}$ & $\sim 5 \mathrm{Myr}$ & $\mathrm{B} 1.5 \mathrm{~V}$ & Howe et al. 1991 \\
\hline & & & & & Wyrowski et al. 2000 \\
\hline \multirow[t]{2}{*}{ NGC2024 } & IRS2b & $360 \mathrm{pc}$ & $\mathrm{emb}$ & $\mathrm{O} 8 \mathrm{~V}$ & Bik et al. 2003 \\
\hline & & & & & Lenorzer et al. 2004 \\
\hline \multirow[t]{2}{*}{$\sigma$ Ori } & $\sigma$ Ori $\mathrm{AB}$ & $350 \mathrm{pc}$ & $\sim 3 \mathrm{Myr}$ & $09.5 \mathrm{~V}$ & Sanz-Forcada et al. 2004 \\
\hline & & & & & Hernandez et al. 2007 \\
\hline \multirow[t]{2}{*}{ Coll69 } & $\lambda$ Ori & $400 \mathrm{pc}$ & $\sim 6 \mathrm{Myr}$ & O8III & Dolan \& Mathieu 2002 \\
\hline & & & & & Barrado y Navascués 2005 \\
\hline \multirow[t]{2}{*}{ S106 } & IRS4 & $600 \mathrm{pc}$ & $\mathrm{emb}$ & O9V & Hodapp \& Rayner 1991 \\
\hline & & & & & Furuya et al. 1999 \\
\hline \multirow[t]{2}{*}{ W40 } & OS1a, 2a, 3a & $600 \mathrm{pc}$ & $\operatorname{obsc}^{b}$ & O9V & Smith et al. 1985 \\
\hline & & & & & Vallée \& MacLeod 1994 \\
\hline
\end{tabular}




\begin{tabular}{|c|c|c|c|c|c|}
\hline Region & Sources & Distance & Age & $\mathrm{L} / \mathrm{SpT}$ & References \\
\hline \multirow[t]{2}{*}{ NGC1579 } & $\mathrm{LkH} \alpha 101$ & $700 \mathrm{pc}$ & obsc & $\mathrm{B} 0.5 \mathrm{~V}$ & Barsony et al. 1991 \\
\hline & & & & & Herbig et al. 2004 \\
\hline \multirow[t]{2}{*}{ NGC2264 } & IRS1 & $800 \mathrm{pc}$ & emb & $\mathrm{B} 2 \mathrm{~V}$ & Schwartz et al. 1985 \\
\hline & & & & & Schreyer et al. 2003 \\
\hline \multirow[t]{2}{*}{ Mon R2 } & IRS3 & $830 \mathrm{pc}$ & $\mathrm{emb}$ & $\mathrm{B} 1 \mathrm{~V}$ & Carpenter et al. 1997 \\
\hline & & & & & Preibisch et al. 2002 \\
\hline \multirow[t]{2}{*}{ GGD 12-15 } & IRAS06084 & $830 \mathrm{pc}$ & $\mathrm{emb}$ & $\mathrm{B} 0.5 \mathrm{~V}$ & Gomez et al. 1998 \\
\hline & & & & & Gutermuth et al. 2005 \\
\hline \multirow[t]{2}{*}{ S140 } & IRS1 & $900 \mathrm{pc}$ & $\mathrm{emb}$ & $\mathrm{B} 2 \mathrm{~V}$ & Lester et al. 1986 \\
\hline & & & & & Preibisch et al. 2001 \\
\hline \multirow[t]{2}{*}{ IC 1396} & HD 206267 & $800 \mathrm{pc}$ & $\sim 3 \mathrm{Myr}$ & $\mathrm{O} 6.5 \mathrm{~V}$ & Schulz et al. 1997 \\
\hline & & & & & de Zeeuw et al. 1999 \\
\hline \multirow[t]{2}{*}{ Cep-OB3 } & Cep-A/HW2 & $725 \mathrm{pc}$ & $\mathrm{emb}$ & $2 \times 10^{4} \mathrm{~L}_{\odot}$ & Garay et al. 1996 \\
\hline & & & & & Hartigan et al. 2000 \\
\hline \multirow[t]{2}{*}{ Cyg-X } & AFGL 2591 & $1000 \mathrm{pc}$ & $\mathrm{emb}$ & $2 \times 10^{4} \mathrm{~L}_{\odot}$ & Tamura \& Yamashita 1992 \\
\hline & & & & & van der Tak et al. 2006 \\
\hline
\end{tabular}

${ }^{a}$ emb stands for embedded, i.e., a very young phase $(<1 \mathrm{Myr})$; sources optically invisible

${ }^{b}$ obsc stands for obscured, i.e., a young phase ( $\left.\sim 1 \mathrm{Myr}\right)$; sources optically visible but heavily dust extincted

Note: AFGL 2591 may be at a distance of 1700 pc (Schneider et al. 2006) and part of the Cyg OB2 association, one of the richest regions of massive stars in the Galaxy with $\sim 100 \mathrm{O}$ stars, including a hidden very massive OB cluster, shown in Figure 18. Note that this massive star forming region is a source of significant diffuse $\gamma$ ray emission, i.e. the $1.8 \mathrm{MeV}$ line from the radioactive decay of ${ }^{26} \mathrm{Al}$ (Plüschke et al. 2002) 


\section{GLOSSARY}

- Accretion

gas accumulation of a star or protostar, increasing the mass of the object

- Competitive accretion

gas initially unbound to a star or protostar and moving with relative speed, $\mathrm{v}_{\text {rel }}$, w.r.t. to the object is added to it, i.e., to its gravitational sphere or column of influence (defined by an impact parameter $2 \mathrm{GM} / \mathrm{v}_{\text {rel }}^{2}$ )

- Coalescence

growth of the mass of a stellar or protostellar object by a physical collision and merger with another object

- Merger

inelastic collision between two stars or protostars leading to the amalgamation of the two bodies

- Massive star

star more massive than about $8 \mathrm{M}_{\odot}$ that ends its life with a type II supernova (unless it is in a close binary system with mass transfer); a massive star on the main sequence has spectral type B3 or earlier.

- OB star

star of spectral type $\mathrm{O}\left(>16 \mathrm{M}_{\odot}\right)$ or type $\mathrm{B}\left(\mathrm{B}>4 \mathrm{M}_{\odot}\right)$

- Ultracompact HII region

small blob (typical density $10^{4} \mathrm{~cm}^{-3}$, size $0.1 \mathrm{pc}$ ) of ionized gas emitting radio continuum radiation of high emission measure $\left(\mathrm{L} \mathrm{n}_{\mathrm{e}}^{2}=10^{7} \mathrm{pc} \mathrm{cm}^{-6}\right)$

- Hypercompact HII region

very small blob (density $10^{6} \mathrm{~cm}^{-3}$, typical size $0.01 \mathrm{pc}$ ) of ionized gas emitting radio continuum radiation of very high emission measure $\left(\mathrm{L} \mathrm{n}_{\mathrm{e}}^{2}=10^{10} \mathrm{pc} \mathrm{cm}^{-6}\right)$

- Protostar

object on its way to become a star, with more than half of its final mass still to be accumulated 
- Infrared Dark Cloud (IRDC)

dense interstellar cloud (often filamentary) seen in absorption against background thermal IR emission; detected with ISO, MSX, and Spitzer

- Core

basic molecular cloud unit (small dense gas fragment, mass $10-100 \mathrm{M}_{\odot}$, size $\sim 0.1 \mathrm{pc}$ ) to form one or a few stars

- Clump

basic molecular cloud unit (large dense gas fragment, mass $\sim 1000-5000 \mathrm{M}_{\odot}$, size $\sim 0.5 \mathrm{pc}$ ) to form a young cluster of stars

- Hot Molecular Core (HMC)

dense warm $(\mathrm{T}>100 \mathrm{~K})$ molecular gas in emission $\left(\mathrm{CO}, \mathrm{NH}_{3}, \mathrm{CH}_{3} \mathrm{OH}\right)$

heated from a protostar inside; a compact region (size $<0.1 \mathrm{pc}$ ) in a star

forming molecular clump such as the BN/KL region in Orion

- Orion Nebula Cluster (ONC)

elongated $1 \times 2$ pc cluster of young stars, centered on the high-mass Trapezium stars in Orion; about 4000 cluster members with a total stellar mass of $\sim 1800 \mathrm{M}_{\odot}$

- Trapezium Cluster

The 4-5 stars in the center of the Orion nebula, $\theta^{1} \mathrm{C}, \mathrm{A}, \mathrm{B}, \mathrm{D}, \mathrm{E}$ in order of decreasing luminosity, the main exciting/ionising source being $\theta^{1}$ $\mathrm{C}\left(\mathrm{O} 5.5 \mathrm{~V}\right.$, ca. $\left.35 \mathrm{M}_{\odot}\right)$

- 30 Doradus (30 Dor) prime example of a giant (200 pc) extragalactic HII region, powered by $\sim 100$ massive O stars stars in the NGC 2070 cluster (cluster mass some $10^{4} \mathrm{M}_{\odot}$, age $<3 \mathrm{Myr}$, cluster radius about 1 arcmin or $15 \mathrm{pc}$ )

- R136

nominally the 1 arcsec core of the 30 Dor nebula and HII region (Radcliffe object No. 136), but R136 also often designates the central dense core of 1 pc ( 4 arcsec) radius of the exciting star cluster NGC 2070 
- Jeans mass

critical mass that must be exceeded for a gas cloud to collapse dynamically, with the self-gravity of the gas cloud being opposed only by thermal pressure; if squeezed by an external pressure, the critical mass for the onset of collapse is called the Bonnor-Ebert mass. Other anisotropic forces such as rotation or magnetic fields can oppose the cloud's self-gravity and thus impede star formation.

- Free-fall timescale

timescale it takes for a pressure-free cloud to collapse dynamically to a very small size (nominally a point) under its own self-gravity; the timescale depends on the average cloud gas density only (for number density $\mathrm{n}=10^{5} \mathrm{~cm}^{-3}$, $\left.\mathrm{t}_{\mathrm{ff}}=10^{5} \mathrm{yr}\right)$.

- Kelvin-Helmholtz (KH) timescale time it takes for a young stellar object or stellar core to radiate its thermal energy content, typically $10^{4} \mathrm{yr}$ for young high-mass stars but $10^{7} \mathrm{yr}$ for solar-type stars $\left(\mathrm{t}_{\mathrm{KH}}=\mathrm{GM}^{2} / \mathrm{RL}\right)$. Nuclear energy generation eventually offsets the thermal energy loss.

- Premain-Sequence evolution

While high-mass stars are born on the Main-Sequence (i.e. they reach hydrogen burning while still accreting matter onto their stellar surface during their deeply embedded phase), the collapse of a low-mass star does not immediately lead to a hydrogen-burning star, rather to a quasi-static object that keeps contracting in radius by factors of a few before hydrogen burning ignites in the center. During this contraction, the young stellar object has a time-dependent radius, effective temperature, and luminosity that can be calculated by pre-MS evolutionary models.

\section{- ZAMS}

zero-age Main Sequence: a star that has contracted enough to start hydrogen burning in its central region

- Initial Mass Function (IMF)

mass distribution of (single) stars at birth introduced by Salpeter in 1955 
- Spitzer

NASA mid-infrared space telescope of $85 \mathrm{~cm}$ diameter launched in 2003

- $M S X$

mid-infrared satellite explorer with an aperture of $20 \mathrm{~cm}$ diameter

- Chandra

$0.5-10 \mathrm{keV}$ NASA X-ray telescope in space with particularly good imaging quality comparable to optical seeing (angular resolution $\sim 1$ arcsec)

- $H S T$

Hubble Space Telescope, $2.4 \mathrm{~m}$ aperture, workhorse for high angular resolution space astronomy in UV, optical, and near-IR; in orbit since 1990 


\section{Acknowledgments}

The present review benefited greatly from insightful discussions with John Bally, Henrik Beuther, Adriaan Blaauw, Ian Bonnell, Bernhard Brandl, Wolfgang Brandner, Anthony Brown, Ed Churchwell, Paul Crowther, Melvyn Davies, Lise Deharveng, Bruce Elmegreen, Neal Evans, Simon Glover, Wolf-Rainer Hamann, Thomas Henning, George Herbig, David Hollenbach, Jim Jackson, Eric Keto, Spyros Kitsionas, Ralf Klessen, Pavel Kroupa, Mark Krumholz, Richard Larson, Hendrik Linz, Andre Maeder, Eric Mamajek, Jorge Melnick, Karl Menten, Vincent Minier, Tony Moffat, Dieter Nürnberger, Tolya Piskunov, Simon Portegies Zwart, Thomas Preibisch, Gregor Rauw, Hugues Sana, Fernando Selman, Joe Silk, Nathan Smith, Bringfried Stecklum, Jonathan Tan, Neal Turner, Virpi Niemela (deceased), Nolan Walborn, and Gerd Weigelt. We thank Geoffrey Burbidge and Allan Sandage for their vote of confidence, and Ewine van Dishoeck and particularly John Kormendy for their critical reading of the manuscript.

Portions of this research was conducted at the Jet Propulsion Laboratory, California Institute of Technology, which is supported by the National Aeronautics and Space Administration (NASA). The assistance of U. Hanschur at AIP Potsdam was indispensible in completing this review and is gratefully acknowledged. H.Z. would like to thank Andrea Lagarini and the Magalhaes family in Rio de Janeiro and ESO in Santiago de Chile for their hospitality during the very final stages of writing this review. This collaboration has a long history, dating back to 1990-1995 when the authors were colocated at the University of Würzburg. 


\section{References}

[1] Aarseth SJ. 2003. Gravitational N-Body Simulations. Cambridge, UK: Cambridge Univ. Press

[2] Abel T, Bryan GL, Norman ML. 2000. Ap. J. 540:39-44

[3] Adams FC, Fatuzzo M. 1996. Ap. J. 464:256-71

[4] Allen DA, Burton MG. 1993. Nature. 363:54-56

[5] Allen C, Poveda A, Hernández-Alcántara A. 2004. Rev. Mex. Astron. Astrofis. Ser. Conf. 21:195-99

[6] Alves J, Lombardi M, Lada CJ. 2007. Astron. Astrophys.Lett. 462:L17-21

[7] Antokhina EA, Moffat AFJ, Antokhin II, Bertrand J-F, Lamontagne R. 2000. Ap. J. 529:463-76

[8] Apai D, Bik A, Kaper L, Henning T, Zinnecker H. 2007. Ap. J. 655:484-91

[9] Appenzeller I. 1970. Astron. Astrophys. 9:216-20

[10] Arce HG, Shepherd D, Gueth F, Lee C-F, Bachiller R, et al. 2007. See Reipurth et al. 2007, pp. 245-60

[11] Balbus SA. 2003. Annu. Rev. Astron. Astrophys. 41:555-97

[12] Balbus SA, Hawley JF. 1991. Ap. J. 376:214-22

[13] Baldry IK, Glazebrook K. 2003. Ap. J. 593:258-71

[14] Ballesteros-Paredes J, Hartmann L, Vázquez-Semadeni E. 1999. Ap. J. 527:285-97

[15] Bally J. 2002. See Crowther 2002, pp. 219-33

[16] Bally J, Moeckel N, Throop H. 2005. In Chondrites and the Protoplanetary Disk, ed. AN Krot, ERD Scott, B Reipurth. ASP Conf. Ser. 341:81-106

[17] Bally J, Cunningham N, Moeckel N, Smith N. 2005. See Cesaroni et al. 2005a, pp. 12-22 
[18] Bally J, Zinnecker H. 2005. Astron. J. 129:2281-93

[19] Banerjee R, Pudritz RE, Anderson DW. 2006. MNRAS 373:1091-06

[20] Banerjee R, Pudritz RE. 2007. Ap. J. 660:479-88

[21] Baraffe I, Chabrier G, Allard F, Hauschildt PH. 2002. Astron. Astrophys. $382: 563-72$

[22] Barrado y Navascués D. 2005. Rev. Mex. Astron. Astrofis. Ser. Conf. 24:217-18

[23] Barsony M, Schombert J, Kis-Halas K. 1991. Ap. J. 379:221-31

[24] Bastian N, Goodwin SP. 2006. MNRAS Lett. 369:L9-13

[25] Basu S, Jones CE. 2004. MNRAS Lett. 347:L47-51

[26] Bate MR. 2000. MNRAS 314:33-53

[27] Bate MR, Bonnell IA, Price NM. 1995. MNRAS 277:362-76

[28] Bate MR, Burkert A. 1997. MNRAS 288:1060-72

[29] Bate MR, Bonnell IA. 1997. MNRAS 285:33-48

[30] Bate MR, Bonnell IA, Bromm V. 2002. MNRAS 336:705-13

[31] Bate MR, Bonnell IA. 2005. MNRAS 356:1201-21

[32] Begelman MC. 2001. Ap. J. 551:897-906

[33] Behrend R, Maeder A. 2001. Astron. Astrophys. 373:190-98

[34] Belkus H, Van Bever J, Vanbeveren D. 2007. Ap. J. 659:1576-81

[35] Benjamin RA, Churchwell E, Babler BL, Bania TM, Clemens DP, et al. 2003. Publ. Astron. Soc. Pac. 115:953-64

[36] Bertoldi F, McKee CF. 1992. Ap. J. 395:140-57

[37] Beuther H, Schilke P, Sridharan TK, Menten KM, Walmsley CM, Wyrowski F. 2002. Astron. Astrophys. 383:892-904 
[38] Beuther H, Shepherd DS. 2005. In Cores to Clusters: Star Formation with Next Generation Telescopes, ed. MS Nanda Kumar, M Tafalla, P Caselli. Astrophys. Space Sci. Libr. 324:105-19

[39] Beuther H, Sridharan TK, Saito M. 2005. Ap. J. Lett. 634:L185-88

[40] Beuther H, Churchwell E, McKee CF, Tan JC. 2007. See Reipurth et al. 2007, pp. $165-80$

[41] Bik A, Lenorzer A, Kaper L, Comerón F, Waters LBFM. 2003. Astron. Astrophys. 404:249-54

[42] Bik A, Kaper L, Hanson MM, Smits M. 2005. Astron. Astrophys. 440:12137

[43] Binney J, Tremaine S. 1987. Galactic Dynamics. Princeton, NJ: Princeton Univ. Press. 755 pp.

[44] Blaauw A. 1961. Bull. Astron. Inst. Neth. 15:265-90

[45] Blaauw A. 1964. Annu. Rev. Astron. Astrophys. 2:213-46

[46] Blaauw A. 1983. Irish Astron. J. 16:141-47

[47] Blaauw A. 1991. In The Physics of Star Formation and Early Stellar Evolution, ed. CJ Lada, ND Kylafis. NATO ASI Ser. C, 342:125-54 Dordrecht: Kluwer

[48] Black DC, Bodenheimer P. 1975. Ap. J. 199:619-32

[49] Blaes O, Socrates A. 2003. Ap. J. 595:509-37

[50] Blandford RD, Payne DG. 1982. MNRAS 199:883-903

[51] Blum RD. 2005. See Cesaroni et al. 2005a, pp. 216-24

[52] Bodenheimer P. 1995. Annu. Rev. Astron. Astrophys. 33:199-238

[53] Bodenheimer P, Laughlin GP, Różyczka M, Yorke HW. 2007. Numerical Methods in Astrophysics. An Introduction. New York: Taylor \& Francis 
[54] Boffin HMJ, Watkins SJ, Bhattal AS, Francis N, Whitworth AP. 1998. MNRAS 300:1189-204

[55] Bonanos AZ. 2007. Astron. J. 133:2696-708

[56] Bonanos AZ, Stanek KZ, Udalski A, Wyrzykowski L, Żebruń K, et al. 2004. Ap. J. Lett. 611:L33-36

[57] Bonnell I, Bastien P. 1992. Ap. J. 401:654-66

[58] Bonnell IA, Bate MR, Clarke CJ, Pringle JE. 1997. MNRAS 285:201-08

[59] Bonnell IA, Bate MR, Zinnecker H. 1998. MNRAS 298:93-102

[60] Bonnell IA, Davies MB. 1998. MNRAS 295:691-98

[61] Bonnell IA, Bate MR, Clarke CJ, Pringle JE. 2001a. MNRAS 323:785-94

[62] Bonnell IA, Clarke CJ, Bate MR, Pringle JE. 2001b. MNRAS 324:573-79

[63] Bonnell IA, Bate MR. 2002. MNRAS 336:659-69

[64] Bonnell IA, Bate MR, Vine SG. 2003. MNRAS 343:413-18

[65] Bonnell IA, Vine SG, Bate MR. 2004. MNRAS 349:735-41

[66] Bonnell IA, Bate MR. 2005. MNRAS 362:915-20

[67] Bonnell IA, Bate MR. 2006. MNRAS 370:488-94

[68] Bonnell IA, Larson RB, Zinnecker H. 2007. See Reipurth et al. 2007, pp. $149-64$

[69] Bosch G, Selman F, Melnick J, Terlevich R. 2001. Astron. Astrophys. 380:137-41

[70] Boss AP, Yorke HW. 1995. Ap. J. Lett. 439:L55-58

[71] Brandl B, Sams BJ, Bertoldi F, Eckart A, Genzel R, et al. 1996. Ap. J. $466: 254-73$

[72] Brandl B, Brandner W, Eisenhauer F, Moffat AFJ, Palla F, Zinnecker H. 1999. Astron. Astrophys. Lett. 352:L69-72 
[73] Brandl BR, Townsley LK, Churchwell E, Carey S, Zinnecker H, et al. 2005. See Cesaroni et al. 2005a, pp. 311-17

[74] Brandner W, Grebel EK, Barbá RH, Walborn NR, Moneti A. 2001. Astron. J. 122:858-65

[75] Brandner W, Clark JS, Stolte A, Waters R, Neguerela I, Goodwin SP. 2007. Astron. Astrophys. In press

[76] Briceño C, Preibisch T, Sherry WH, Mamajek EA, Mathieu RD, et al. 2007. See Reipurth et al. 2007, pp. 345-60

[77] Bromm V, Larson RB. 2004. Annu. Rev. Astron. Astrophys. 42:79-118

[78] Bronfman L, Casassus S, May J, Nyman L-Å. 2000. Astron. Astrophys. 358:521-34

[79] Brown A. 2001. Astron. Nachr. 322:43-46

[80] Burrows CJ, Stapelfeldt KR, Watson AM, Krist JE, Ballester GE, et al. 1996. Ap. J. 473:437-51

[81] Burton MG, Hill T, Longmore SN, Purcell CR, Walsh AJ. 2005. See Cesaroni et al. 2005a, pp. 157-62

[82] Camenzind M. 1990. Rev. Mod. Astron. 3:234-65

[83] Carpenter JM, Snell RL, Schloerb FP, Skrutskie MF. 1993. Ap. J. 407:65779

[84] Carpenter JM, Meyer MR, Dougados C, Strom SE, Hillenbrand LA. 1997. Astron. J. 114:198-221

[85] Cassinelli JP, Mathis JS, Savage, BD. 1981. Science 212:1497-501

[86] Cassinelli JP, Churchwell EB, ed. 1993. Massive Stars: Their Lives in the Interstellar Medium, ASP Conf. Ser., Vol. 35. San Francisco: ASP

[87] Cesaroni R. 2005. Ap. Space Sci. 295:5-17 
[88] Cesaroni R, Felli M, Testi L, Walmsley CM, Olmi L. 1997. Astron. Astrophys. $325: 725-44$

[89] Cesaroni R, Felli M, Jenness T, Neri R, Olmi L, et al. 1999. Astron. Astrophys. 345:949-64

[90] Cesaroni R, Felli M, Churchwell E, Walmsley M, ed. 2005a. Massive Star Birth: A Crossroads of Astrophysics, IAU Symp. 22\%. Cambridge: Cambridge University Press

[91] Cesaroni R, Neri R, Olmi L, Testi L, Walmsley CM, Hofner P. 2005b. Astron. Astrophys. 434:1039-54

[92] Cesaroni R, Galli D, Lodato G, Walmsley CM, Zhang Q. 2007. See Reipurth et al. 2007, pp. 197-212

[93] Chabrier G. 2003. Publ. Astron. Soc. Pac. 115:763-95

[94] Chini R, Hoffmeister V. Kimeswenger S, Nielbock M, Nürnberger D, et al. 2004. Nature 429:155-57

[95] Churchwell E. 2002. Annu. Rev. Astron. Astrophys. 40:27-62

[96] Churchwell E, Povich MS, Allen D, Taylor MG, Meade MR, et al. 2006. Ap. J. 649:759-78

[97] Clark JS, Negueruela I, Crowther PA, Goodwin SP. 2005. Astron. Astrophys. 434:949-69

[98] Clark PC, Bonnell IA. 2005. MNRAS 361:2-16

[99] Clark PC, Bonnell IA, Zinnecker H. 2005. MNRAS 359:809-18

[100] Clarke CJ, Pringle JE. 1992. MNRAS 255:423-30

[101] Clarke CJ, Edgar RG, Dale JE. 2005. See Corbelli et al. 2005, pp. 449-54

[102] Comerón F, Schneider N, Russeil D. 2005. Astron. Astrophys. 433:955-77

[103] Comerón F, Pasquali A. 2007. Astron. Astrophys. Lett. 467:L23-27 
[104] Corbelli E, Palla F, Zinnecker H. ed. 2005. The Initial Mass Function 50 Years Later, Astrophys. Space Sci. Libr. 327. Dordrecht: Springer

[105] Costero R, Echevarria J, Richer MG, Poveda A, Li W. 2006. IAU Circ. 8669

[106] Crowther PA, ed. 2002. Hot Star Workshop III: The Earliest Stages of Massive Star Birth, ASP Conf. Proc., Vol. 267. San Francisco: ASP

[107] Crowther PA. 2007. Annu. Rev. Astron. Astrophys. 45:In press

[108] Crutcher RM. 1999. Ap. J. 520:706-13

[109] Crutcher RM. 2005. See Cesaroni et al. 2005a, pp. 98-107

[110] Crutcher RM, Troland TH. 2007. See Elmegreen, Palous. 2007, pp. 141-47

[111] Dale JE, Davies MB. 2006. MNRAS 366:1424-36

[112] Dale JE, Bonnell IA, Whitworth AP. 2007. MNRAS 375:1291-98

[113] Dale JE, Clark PC, Bonnell IA. 2007. MNRAS 377:535-44

[114] Davies MB, Bate MR, Bonnell IA, Bailey VC, Tout CA. 2006. MNRAS 370:2038-46

[115] Davis CJ, Varricatt WP, Todd SP, Ramsay Howat SK. 2004. Astron. Astrophys. 425:981-95

[116] De Becker M, Rauw G, Manfroid J, Eenens P. 2006. Astron. Astrophys. 456:1121-30

[117] De Buizer JM. 2003. MNRAS 341:277-98

[118] De Buizer JM, Minier V. 2005. Ap. J. Lett. 628:L151-54

[119] de Geus EJ. 1992. Astron. Astrophys. 262:258-70

[120] de Wit WJ, Testi L, Palla F, Vanzi L, Zinnecker H. 2004. Astron. Astrophys. 425:937-48 
[121] de Wit WJ, Testi L, Palla F, Zinnecker H. 2005. Astron. Astrophys. $437: 247-55$

[122] de Zeeuw PT, Hoogerwerf R, de Bruijne JHJ, Brown AGA, Blaauw A. 1999. Astron. J. 117:354-99

[123] Diehl R, Halloin H, Kretschmer K, Lichti GG, Schönfelder V, et al. 2006. Nature 439:45-47

[124] Dobbs CL, Bonnell IA, Clark PC. 2005. MNRAS 360:2-8

[125] Dolan CJ, Mathieu RD. 2002. Astron. J. 123:387-403

[126] Donati J-F, Babel J, Harries TJ, Howarth ID, Petit P, Semel M. 2002. MNRAS 333:55-70

[127] Donati J-F, Howarth ID, Bouret J-C, Petit P, Catala C, Landstreet J. 2006. MNRAS Lett. 365:L6-10

[128] Drissen L, Moffat AFJ, Walborn NR, Shara MM. 1995. Astron. J. 110:2235-41

[129] Duchêne G, Simon T, Eisloffel J, Bouvier J. 2001. Astron. Astrophys. 379:147-61

[130] Durisen RH. 2001. See Zinnecker, Mathieu. 2001, pp. 381-90

[131] Edgar R, Clarke C. 2004. MNRAS 349:678-86

[132] Edwards S, Strom SE, Hartigan P, Strom KM, Hillenbrand LA, et al. 1993. Astron. J. 106:372-82

[133] Egan MP, Shipman RF, Price SD, Carey SJ, Clark FO, Cohen M. 1998. Ap. J. Lett. 494:L199-202

[134] Eikenberry SS, Matthews K, LaVine JL, Garske MA, Hu D, et al. 2004. Ap. J. $616: 506-18$

[135] Eisenhauer F, Quirrenbach A, Zinnecker H, Genzel R. 1998. Ap. J. 498:278-92 
[136] Elmegreen BG. 1997. Ap. J. 486:944-54

[137] Elmegreen BG. 1998. In The Origin of Stars and Planetary Systems, ed. CE Woodward, JM Shull, HA Thronson Jr. ASP Conf. Ser., 148:150-83

[138] Elmegreen BG. 1999. Ap. J. 517:103-07

[139] Elmegreen BG. 2000. Ap. J. 539:342-51

[140] Elmegreen BG, Lada CJ. 1977. Ap. J. 214:725-41

[141] Elmegreen BG, Palous J. ed. 2007. Triggered Star Formation in a Turbulent ISM, IAU Symp. 23\%. Cambridge: Cambridge University Press

[142] Evans NJ. 1999. Annu. Rev. Astron. Astrophys. 37:311-62

[143] Evans NJ. 2005. See Cesaroni et al. 2005a, pp. 443-48

[144] Fabian AC, Pringle JE, Rees MJ. 1975. MNRAS 172:15-18

[145] Feitzinger JV, Schlosser W, Schmidt-Kaler T, Winkler C. 1980. Astron. Astrophys. 84:50-59

[146] Ferreira J, Pelletier G. 1995. Astron. Astrophys. 295:807-32

[147] Figer DF. 2003. In A Massive Star Odyssey: From Main Sequence to Supernova, ed. K van der Hucht, A Herrero, E César. IAU Symp. 212:487-96. San Francisco: ASP

[148] Figer DF. 2005. Nature 434:192-94

[149] Figer DF, Najarro F, Morris M, McLean IS, Geballe TR, et al. 1998. Ap. J. 506:384-404

[150] Figer DF, Najarro F, Kudritzki RP. 2004. Ap. J. Lett. 610:L109-12

[151] Fromang S, Balbus S, Terquem C, de Villiers JP. 2004. Ap. J. 616:364-75

[152] Fryer CL, Heger A. 2005. Ap. J. 623:302-13

[153] Furuya RS, Kitamura Y, Saito M, Kawabe R, Wootten HA. 1999. Ap. J. $525: 821-31$ 
[154] Gammie CF. 1998. MNRAS 297:929-35

[155] Garay G. 2005. See Cesaroni et al. 2005a, pp. 86-91

[156] Garay G, Ramirez S, Rodríguez LF, Curiel S, Torrelles JM. 1996. Ap. J. 459:193-208

[157] Garay G, Lizano S. 1999. Publ. Astron. Soc. Pac. 111:1049-87

[158] Garay G, Brooks KJ, Mardones D, Norris RP. 2003. Ap. J. 587:739-47

[159] Garay G, Faúndez S, Mardones D, Bronfman L, Chini R, Nyman L-Å. 2004. Ap. J. 610:313-19

[160] García B, Mermilliod JC. 2001. Astron. Astrophys. 368:122-36

[161] Garmany CD. 1994. Publ. Astron. Soc. Pac. 106:25-37

[162] Garmany CD, Conti PS, Massey P. 1980. Ap. J. 242:1063-76

[163] Garmany CD, Conti PS, Chiosi C. 1982. Ap. J. 263:777-90

[164] Genzel R, Stutzki J. 1989. Annu. Rev. Astron. Astrophys. 27:41-85

[165] Gerola H, Seiden PE. 1978. Ap. J. 223:129-35

[166] Gies DR. 1987. Ap. J. Suppl. 64:545-63

[167] Gies DR, Bolton CT. 1986. Ap. J. Suppl. 61:419-54

[168] Glover SCO. 2005. Space Sci. Rev. 117:445-508

[169] Glover SCO, Mac Low M-M. 2007. Ap. J. 659:1317-37

[170] Gomez Y, Lebron M, Rodríguez LF, Garay G, Lizano S, et al. 1998. Ap. J. 503:297-306

[171] Greenhill LJ, Gezari DY, Danchi WC, Najita J, Monnier JD, Tuthill PG. 2004. Ap. J. Lett. 605:L57-60

[172] Gualandris A, Portegies Zwart S, Eggleton PP. 2004. MNRAS 350:615-26

[173] Güsten R, Mezger PG. 1982. Vistas Astron. 26:159-224 
[174] Gueth F, Guilloteau S. 1999. Astron. Astrophys. 343:571-84

[175] Gutermuth RA, Megeath ST, Pipher JL, Williams JP, Allen LE, et al. 2005. Ap. J. 632:397-420

[176] Habing HJ, Israel FP. 1979. Annu. Rev. Astron. Astrophys. 17:345-85

[177] Hartigan P, Morse J, Bally J. 2000. Astron. J. 120:1436-48

[178] Hawley JF, Balbus SA. 1991. Ap. J. 376:223-33

[179] Heger A, Fryer CL, Woosley SE, Langer N, Hartmann DH. 2003. Ap. J. 591:288-300

[180] Heitsch F, Mac Low M-M, Klessen RS. 2001. Ap. J. 547:280-91

[181] Heller CH. 1995. Ap. J. 455:252-59

[182] Henning T, Schreyer K, Launhardt R, Burkert A. 2000. Astron. Astrophys. $353: 211-26$

[183] Henning T, Stecklum B. 2002. In Modes of Star Formation and the Origin of Field Populations, ed. EK Grebel, W Brandner. ASP Conf. Proc. 285:40-48

[184] Henriksen RN. 1991. Ap. J. 377:500-09

[185] Herbig GH, Andrews SM, Dahm SE. 2004. Astron. J. 128:1233-53

[186] Herbig GH, Griffin RF. 2006. Astron. J. 132:1763-67

[187] Herbst W, Assousa GE. 1977. Ap. J. 217:473-75

[188] Hernandez J, Hartmann L, Megeath T, Gutermuth R, Muzerolle J, et al. 2007. Ap. J. In press (astro-ph/0701476)

[189] Heydari-Malayeri M, Rosa MR, Schaerer D, Martins F, Charmandaris V. 2002. Astron. Astrophys. 381:951-58

[190] Heydari-Malayeri M, Meynadier F, Charmandaris V, Deharveng L, Le Bertre T, et al. 2003. Astron. Astrophys. 411:427-35 
[191] Hill T, Burton MG, Minier V, Thompson MA, Walsh AJ, et al. 2005. MNRAS 363:405-51

[192] Hillenbrand LA. 1997. Astron. J. 113:1733-68

[193] Hillenbrand LA, Hartmann LW. 1998. Ap. J. 492:540-53

[194] Hoare MG, Kurtz SE, Lizano S, Keto E, Hofner P. 2007. See Reipurth et al. 2007, pp. $181-96$

[195] Hodapp K-W, Rayner J. 1991. Astron. J. 102:1108-17

[196] Hofmann K-H, Seggewiss W, Weigelt G. 1995. Astron. Astrophys. 300:40314

[197] Hofner P, Delgado H, Whitney B, Churchwell E, Linz H. 2002. Ap. J. Lett. 579:L95-98

[198] Hollenbach D, Johnstone D, Lizano S, Shu F. 1994. Ap. J. 428:654-69

[199] Hollenbach D, Yorke HW, Johnstone D. 2000. See Mannings et al. 2000, pp. $401-28$

[200] Hoogerwerf R, de Bruijne JHJ, de Zeeuw PT. 2001. Astron. Astrophys. $365: 49-77$

[201] Howe JE, Jaffe DT, Genzel R, Stacey GJ. 1991. Ap. J. 373:158-68

[202] Hunter DA. 1995. In Gaseous Nebulae and Star Formation. Rev. Mex. Astron. Astrofis. Ser. Conf. 3:1-7

[203] Indebetouw R, Whitney BA, Johnson KE, Wood K. 2006. Ap. J. 636:36280

[204] Irvine NJ. 1989. Ap. J. Lett. 337:L33-35

[205] Jappsen A-K, Klessen RS, Larson RB, Li Y, Mac Low M-M. 2005. Astron. Astrophys. 435:611-23

[206] Jijina J, Adams FC. 1996. Ap. J. 462:874-87

[207] Johnstone, Doug; Bally, John. 2006. Ap. J. 653:383-97 
[208] Kahn FD. 1974. Astron. Astrophys. 37:149-62

[209] Kaifu N, Usuda T, Hayashi SS, Itoh Y, Akiyama M, et al. 2000. Publ. Astron. Soc. Jpn. 52:1-8

[210] Kastner JH, Buchanan CL, Sargent B, Forrest WJ. 2006. Ap. J. Lett. 638:L29-32

[211] Kennicutt RC. 1998. Annu. Rev. Astron. Astrophys. 36:189-232

[212] Kennicutt RC. 2005. See Cesaroni et al. 2005a, pp. 3-11

[213] Kessel-Deynet O, Burkert A. 2003. MNRAS 338:545-54

[214] Keto E. 2002. Ap. J. 580:980-86

[215] Keto E. 2007. Ap. J. In press (astro-ph/0603856)

[216] Keto E, Wood K. 2006. Ap. J. 637:850-59

[217] Kharchenko NV, Piskunov AE, Röser S, Schilbach E, Scholz R-D. 2004. Astron. Nachr. 325:740-48

[218] Kharchenko NV, Piskunov AE, Röser S, Schilbach E, Scholz R-D. 2005. Astron. Astrophys. 440:403-08

[219] Kim SS, Figer DF, Kudritzki RP, Najarro F. 2006. Ap. J. Lett. 653:L11316

[220] Kippenhahn R, Meyer-Hofmeister E. 1975. Astron. Astrophys. 54:539-42

[221] Kitsionas S, Whitworth AP. 2002. MNRAS 330:129-36

[222] Klein RI, Fisher R, McKee CF. 2004. In Gravitational Collapse: From Massive Stars to Planets, ed. G Garcia-Segura, G Tenorio-Tagle, J Franco, HW Yorke. Rev. Mex. Astron. Astrophys. Ser. Conf., 22:3-7

[223] Klessen RS. 2001a. Ap. J. Lett. 550:L77-80

[224] Klessen RS. 2001b. Ap. J. 556:837-46

[225] Klessen RS, Burkert A. 2001. Ap. J. 549:386-401 
[226] Klessen RS, Ballesteros-Paredes J, Vázquez-Semadeni E, Durán-Rojas C. 2005. Ap. J. 620:786-94

[227] Klessen RS, Spaans M, Jappsen A-K. 2007. MNRAS Lett. 374:L29-33

[228] Knodlseder J. 2000. Astron. Astrophys. 360:539-48

[229] Koen C. 2006. MNRAS 365:590-94

[230] Konigl A. 1991. Ap. J. Lett. 370:L39-43

[231] Kratter KM, Matzner CD. 2006. MNRAS. 373:1563-76

[232] Kraus S, Balega YY, Berger J-P, Hofmann K-H, Millan-Gabet R, et al. 2007. Astron. Astrophys. 466:649-59

[233] Kritsuk AG, Norman ML, Padoan P. 2006. Ap. J. 638:L25-28

[234] Kroupa P. 2000. In Massive Stellar Clusters, ed. A Lancon, C Boily. ASP Conf. Ser., 211:233-40

[235] Kroupa P. 2002. Science 295:82-91

[236] Kroupa P, Aarseth S, Hurley J. 2001, MNRAS 321:699-712

[237] Krumholz MR. 2006. Ap. J. Lett. 641:L45-48

[238] Krumholz MR. 2007. In Massive Stars: From Pop III and GRBs to the Milky Way, ed. M Livio, E Villaver. In press (astro-ph/0607429)

[239] Krumholz MR, McKee CF, Klein RI. 2004. Ap. J. 611:399-412

[240] Krumholz MR, Klein RI, McKee CF. 2005. See Cesaroni et al. 2005a, pp. $231-36$

[241] Krumholz MR, McKee CF, Klein RI. 2005a. Ap. J. 618:L33-36

[242] Krumholz MR, McKee CF, Klein RI. 2005b. Nature 438:332-34

[243] Krumholz MR, Klein RI, McKee CF. 2007. Ap. J. 656:959-79

[244] Krumholz MR, Thompson TA. 2007. Ap. J. In press (astro-ph/0611822) 
[245] Kudritzki R. 2002. Ap. J. 577:389-408

[246] Kurtz S. 2005. See Cesaroni et al. 2005a, pp. 111-19

[247] Kurtz S, Cesaroni R, Churchwell E, Hofner P, Walmsley CM. 2000. See Mannings et al. 2000, pp. 299-326

[248] Lada CJ, Thronson HA Jr., Smith HA, Schwartz PR, Glaccum W. 1984. Ap. J. 286:302-09

[249] Lada CJ, Lada EA. 2003. Annu. Rev. Astron. Astrophys. 41:57-115

[250] Lamers HJGLM, Panagia N, Scuderi S, Romaniello M, Spaans M, et al. 2002. Ap. J. 566:818-32

[251] Larson RB. 1969. MNRAS 145:271-95

[252] Larson RB. 1978. MNRAS 184:69-85

[253] Larson RB. 1981. MNRAS 194:809-26

[254] Larson RB. 1982. MNRAS 200:159-74

[255] Larson RB. 1985. MNRAS 214:379-98

[256] Larson RB. 2005. MNRAS 359:211-22

[257] Larson RB, Starrfield S. 1971. Astron. Astrophys. 13:190-97

[258] Laughlin G, Bodenheimer P. 1994. Ap. J. 436:335-54

[259] Ledoux P. 1941. Ap. J. 94:537-38

[260] Lee C-F, Ho PTP, Beuther H, Bourke TL, Zhang Q, et al. 2006. Ap. J. 639:292-302

[261] Lee H-T, Chen WP. 2007. Ap. J. 657:884-96

[262] Lenorzer A, Bik A, de Koter A, Kurtz SE, Waters LBFM, et al. 2004. Astron. Astrophys. 414:245-59

[263] Leonard PJT, Duncan MJ. 1990. Astron. J. 99:608-16 
[264] Lester DF, Harvey PM, Joy M, Ellis HB Jr. 1986. Ap. J. 309:80-89

[265] Li Y. Klessen RS, Mac Low M-M. 2003. Ap. J. 592:975-85

[266] Li Z-Y, Nakamura F. 2006. Ap. J. Lett. 640:L187-90

[267] Linz H, Stecklum B, Henning T, Hofner P, Brandl B. 2005. Astron. Astrophys. 429:903-21

[268] Mac Low M-M, Klessen RS. 2004. Rev. Mod. Phys. 76:125-94

[269] Maeder A, Behrend R. 2002. See Crowther 2002, pp. 179-92

[270] Maiolino R, Vanzi L, Mannucci F, Cresci G, Ghinassi F, Della Valle M. 2002. Astron. Astrophys. 389:84-92

[271] Maíz-Apellániz J, Pérez E, Mas-Hesse JM. 2004. Astron. J. 128:1196-218

[272] Maíz-Apellániz J, Walborn NR, Morrell NI, Niemela VS, Nelan EP. 2007. Ap. J. In press (astro-ph/0612012)

[273] Makinen P, Harvey PM, Wilking BA, Evans NJ II. 1985. Ap. J. 299:34150

[274] Mannings I, Boss AP, Russell SS, ed. 2000. Protostars and Planets IV. Tucson: Univ. Ariz. Press

[275] Martel H, Evans NJ II, Shapiro PR. 2006 Ap. J. Suppl. 163:122-44

[276] Martins F, Schaerer D, Hillier DJ. 2005. Astron. Astrophys. 436:1049-65

[277] Martins F, Trippe S, Paumard T, Ott T, Genzel R, et al. 2006. Ap. J. Lett. 649 :L103-06

[278] Mason BD, Gies DR, Hartkopf WI, Bagnuolo WG Jr., ten Brummelaar T, McAlister HA. 1998. Astron. J. 115:821-47

[279] Massey P. 1998. In The Stellar Initial Mass Function, ed. G Gilmore, D Howell. ASP Conf. Ser., 142:17-44

[280] Massey P. 2002. Ap. J. Suppl. 141:81-122 
[281] Massey P. 2003. Annu. Rev. Astron. Astrophys. 41:15-56

[282] Massey P, Johnson KE, Degioia-Eastwood K. 1995. Ap. J. 454:151-71

[283] Massey P, Hunter DA. 1998. Ap. J. 493:180-94

[284] Massey P, Penny LR, Vukovich J. 2002. Ap. J. 565:982-93

[285] Masunaga H, Miyama SM, Inutsuka S-I. 1998. Ap. J. 495:346-69

[286] Masunaga H, Inutsuka S-I. 2000. Ap. J. 531:350-65

[287] Mathis JS, Rumpl W, Nordsieck KH. 1977. Ap. J. 217:425-33

[288] McCaughrean MJ. 2001. In From Darkness to Light: Origin and Evolution of Young Stellar Clusters, ed. T Montmerle, P André. ASP Conf. Ser., 243:449-60

[289] McCaughrean MJ, Rayner JT, Zinnecker H. 1994. Ap. J. Lett. 436:L189-

92

[290] McCaughrean MJ, Mac Low M-M. 1997. Astron. J. 113:391-400

[291] McKee CF, Tan JC. 2003. Ap. J. 585:850-71

[292] McKee CF, Ostriker EC. 2007. Annu. Rev. Astron. Astrophys. 45:In press [293] McMillan SLW, Vesperini E, Portegies Zwart SF. 2007. Ap. J. Lett. 655:L45-49

[294] Megeath ST, Wilson TL, Corbin MR. 2005. Ap. J. Lett. 622:L141-44

[295] Meixner M, Gordon KD, Indebetouw R, Hora JL, Whitney B, et al. 2006. Astron. J. 132:2268-88

[296] Melioli C, de Gouveia Dal Pino EM, de La Reza R, Raga A. 2006. MNRAS $373: 811-18$

[297] Mengel S. Lehnert MD, Thatte N, Genzel R. 2002. Astron. Astrophys. $383: 137-52$ 
[298] Mengel S, Tacconi-Garman LE. 2007. Astron. Astrophys. In press (astroph/0701415)

[299] Menten KM. 1991. Ap. J. Lett. 380:L75-78

[300] Menten KM, Reid MJ. 1995. Ap. J. Lett. 445:L157-60

[301] Menten KM, Pillai T, Wyrowski F. 2005. See Cesaroni et al. 2005a, pp. $23-34$

[302] Mermilliod J-C, García B. 2001. See Zinnecker, Mathieu. 2001, pp. 191-98

[303] Meynet G, Maeder A. 2003. Astron. Astrophys. 404:975-90

[304] Meynet G, Maeder A. 2005. Astron. Astrophys. 429:581-98

[305] Meynet G, Maeder A, Schaller G, Schaerer D, Charbonnel C. 1994. Astron. Astrophys. Suppl. 103:97-105

[306] Mezger PG, Altenhoff W, Schraml J, Burke BF, Reifenstein EC III, Wilson, TL.1967. Ap. J. 150:L157-66

[307] Minier V, Burton MG, Hill T, Pestalozzi MR, Purcell CR, et al. 2005. Astron. Astrophys. 429:945-60

[308] Moeckel N, Bally J. 2006. Ap. J. 653:437-46

[309] Moeckel N, Bally J. 2007. Ap. J. 656:275-86

[310] Moffat AFJ, Drissen L, Shara MM. 1994. Ap. J. 436:183-93

[311] Moffat AFJ, Poitras V, Marchenko SV, Shara MM, Zurek DR, et al. 2004. Astron. J. 128:2854-61

[312] Monaghan JJ. 1992. Annu. Rev. Astron. Astrophys. 30:543-74

[313] Monaghan JJ. 2005. Rep. Prog. Phys. 68:1703-59

[314] Monin J-L, Clarke CJ, Prato L, McCabe C. 2007. See Reipurth et al. 2007, pp. 395-409

[315] Morrell NI, Levato H. 1991. Ap. J. Suppl. 75:965-85 
[316] Morrell NI, Barbá RH, Niemela VS, Corti MA, Albacete Colombo JF, et al. 2001. MNRAS 326:85-94

[317] Motte F, Bontemps S, Schilke P, Lis DC, Schneider N, Menten KM. 2005. See Cesaroni et al. 2005a, pp. 151-56

[318] Muench AA, Lada EA, Lada CJ, Alves J. 2002. Ap. J. 573:366-93

[319] Nakano T, Hasegawa T, Norman C. 1995. Ap. J. 450:183-95

[320] Nelan EP, Walborn NR, Wallace DJ, Moffat AFJ, Makidon RB, et al. 2004. Astron. J. 128:323-29

[321] Nielbock M, Chini R, Hoffmeister VH, Scheyda CM, Steinacker J, et al. 2007. Ap. J. Lett. 656:L81-84

[322] Norberg P, Maeder A. 2000. Astron. Astrophys. 359:1025-34

[323] Nürnberger DEA. 2003. Astron. Astrophys. 404:255-65

[324] Nürnberger DEA, Chini R, Eisenhauer F, Kissler-Patig M, Modigliani A, et al. 2007. Astron. Astrophys. 465:931-36

[325] Oey MS, Clarke CJ. 2005. Ap. J. Lett. 620:L43-46

[326] Omukai K, Palla F. 2003. Ap. J. 589:677-87

[327] Öpik EJ. 1953. Irish Astron. J. 2:219-33

[328] Padgett DL, Strom SE, Ghez A. 1997. Ap. J. 477:705-10

[329] Padoan P, Nordlund Å. 2002. Ap. J. 576:870-79

[330] Palla F, Stahler SW. 1992. Ap. J. 392:667-77

[331] Palla F, Stahler SW. 1993. Ap. J. 418:414-25

[332] Parker JW, Garmany CD. 1993. Astron. J. 106:1471-83

[333] Patel NA, Curiel S, Sridharan TK, Zhang Q, Hunter TR, et al. 2005. Nature 437:109-11 
[334] Paumard T, Genzel R, Martins F, Nayakshin S, Beloborodov AM, et al. 2006. Ap. J. 643:1011-35

[335] Pehlemann E, Hofmann K-H, Weigelt G. 1992. Astron. Astrophys. 256:701-14

[336] Perault M, Omont A, Simon G, Seguin P, Ojha D, et al. 1996. Astron. Astrophys. Lett. 315:L165-68

[337] Peretto N, André P, Belloche A. 2006. Astron. Astrophys. 445:979-98

[338] Pflamm-Altenburg J, Kroupa P. 2006. MNRAS 373:295-304

[339] Pietrinferni A, Cassisi S, Salaris M, Castelli F. 2004. Ap. J. 612:168-90

[340] Piskunov AE, Kharchenko NV, Röser S, Schilbach E, Scholz RD. 2006. Astron. Astrophys. 445:545-65

[341] Plume R, Jaffe DT, Evans NJ II, Martin-Pintado J, Gomez-Gonzalez J. 1997. Ap. J. 476:730-49

[342] Plüschke S, Cerviño M, Diehl R, Kretschmer K, Hartmann DH, Knödlseder J. 2002. New Astron. Rev. 46:535-39

[343] Poetzel R, Mundt R, Ray TP. 1992. Astron. Astrophys. 262:229-47

[344] Portegies Zwart SF, McMillan SLW. 2002. Ap. J. 576:899-907

[345] Portegies Zwart SF, Baumgardt H, McMillan SLW, Makino J, Hut P, Ebisuzaki T. 2006. Ap. J. 641:319-26

[346] Poveda A, Ruiz J, Allen C. 1967. Bol. Obs. Tonantzintla Tacubaya 4:86-90

[347] Preibisch T, Ossenkopf V, Yorke HW, Henning T. 1993. Astron. Astrophys. 279:577-88

[348] Preibisch T, Balega Y, Hofmann K-H, Weigelt G, Zinnecker H. 1999. New Astron. 4:531-42

[349] Preibisch T, Zinnecker H. 1999. Astron. J. 117:2381-97 
[350] Preibisch T, Weigelt G, Zinnecker H. 2001. See Zinnecker \& Mathieu 2001, p. $69-78$

[351] Preibisch T, Balega YY, Schertl D, Smith MD, Weigelt G. 2001. Astron. Astrophys. 378:539-45

[352] Preibisch T, Balega YY, Schertl D, Weigelt G. 2002. Astron. Astrophys. 392:945-54

[353] Preibisch T, Balega YY, Schertl D, Weigelt G. 2003. Astron. Astrophys. $412: 735-43$

[354] Preibisch T, Zinnecker H. 2007. See Elmegreen, Palous. 2007, pp. 270-77

[355] Pudritz RE, Norman CA. 1983. Ap. J. 274:677-97

[356] Pudritz RE, Ouyed R, Fendt C, Brandenburg A. 2007. See Reipurth et al. 2007, pp. 277-94

[357] Rathborne JM, Jackson JM, Simon R. 2006. Ap. J. 641:389-405

[358] Reipurth B, Zinnecker H. 1993. Astron. Astrophys. 278:81-108

[359] Rauw G, De Becker M, Nazé Y, Crowther PA, Gosset E, et al. 2004. Astron. Astrophys. Lett. 420:L9-13

[360] Reipurth B, Jewitt D, Keil K, ed. 2007. Protostars and Planets V. Tucson: Univ. Ariz. Press

[361] Richer JS, Shepherd DS, Cabrit S, Bachiller R, Churchwell E. 2000. See Mannings et al. 2000, pp. 867-96

[362] Richling S, Yorke HW. 1997. Astron. Astrophys. 327:317-24

[363] Roberts MS. 1957. Publ. Astron. Soc. Pac. 69:59-64

[364] Rodríguez LF, Poveda A, Lizano S, Allen C. 2005a. Ap. J. Lett. 627:L6568

[365] Rodríguez LF, Garay G, Brooks KJ, Mardones D. 2005b. Ap. J. 626:95358 
[366] Rubio M, Barbá RH, Walborn NR, Probst RG, García J, Roth MR. 1998. Astron. J. 116:1708-18

[367] Salpeter EE. 1955. Ap. J. 121:161-67

[368] Sana H, Rauw G, Gosset E. 2005. In Massive Stars and High-Energy Emission in OB Associations, Proc. Workshop JENAM, ed. G Rauw, Y Nazé, R Blomme, E Gosset, pp. 107-10

[369] Sandage A. 1986. Astron. Astrophys. 161:89-101

[370] Sanz-Forcada J, Franciosini E, Pallavicini R. 2004. Astron. Astrophys. $421: 715-27$

[371] Scalo JM. 1998. In The Stellar Initial Mass Function, ed. G Gilmore, D Howell. ASP Conf. Ser. 142:201-36

[372] Schaerer D, de Koter A. 1997. Astron. Astrophys. 322:598-614

[373] Schertl D, Balega YY, Preibisch T, Weigelt G. 2003. Astron. Astrophys. 402:267-75

[374] Schmeja S, Klessen RS. 2004. Astron. Astrophys. 419:405-17

[375] Schmid-Burgk J, Guesten R, Mauersberger R, Schulz A, Wilson TL. 1990. Ap. J. Lett. 362:L25-28

[376] Schneider N, Bontemps S, Simon R, Jakob H, Motte F, et al. 2006. Astron. Astrophys. 458:855-71

[377] Schreyer K, Stecklum B, Linz H, Henning T. 2003. Ap. J. 599:335-41

[378] Schreyer K, Semenov D, Henning T, Forbrich J. 2006. Ap. J. Lett. 637:L129-32

[379] Schulz NS, Berghoefer TW, Zinnecker H. 1997. Astron. Astrophys. $325: 1001-12$

[380] Schwartz PR, Thronson HA Jr., Odenwald SF, Glaccum W, Loewenstein RF, Wolf G. 1985. Ap. J. 292:231-37 
[381] Schwarzschild M, Härm R. 1959. Ap. J. 129:637-46

[382] Schweickhardt J, Schmutz W, Stahl O, Szeifert T, Wolf B. 1999. Astron. Astrophys. 347:127-36

[383] Selman FJ, Melnick J. 2005. Astron. Astrophys. 443:851-61

[384] Sewilo M, Churchwell E, Kurtz S, Goss WM, Hofner P. 2004. Ap. J. 605:285-99

[385] Shepherd D. 2005. See Cesaroni et al. 2005a, pp. 237-46

[386] Shepherd DS, Churchwell E. 1996a. Ap. J. 472:225-39

[387] Shepherd DS, Churchwell E. 1996b. Ap. J. 457:267-76

[388] Shepherd DS, Claussen MJ, Kurtz SE. 2002. See Crowther 2002, pp. 415-

16

[389] Shirley YL, Evans NJ II, Young KE, Knez C, Jaffe DT. 2003. Ap. J. Suppl. 149:375-403

[390] Shu FH. 1977. Ap. J. 214:488-97

[391] Shu FH, Adams FC, Lizano S. 1987. Annu. Rev. Astron. Astrophys. 25:23-

81

[392] Shu FH, Najita J, Ostriker E, Wilkin F, Ruden S, Lizano S. 1994. Ap. J. 429:781-96

[393] Shu FH, Najita J, Ostriker EC, Shang H. 1995. Ap. J. Lett. 459:L155-8

[394] Silk J. 1978. In Protostars and Planets I, ed. T Gehrels, p. 172. Tucson: Univ. Ariz. Press

[395] Silk J. 1995. Ap. J. Lett. 438:L41-44

[396] Silk J. 1997. Ap. J. 481:703-09

[397] Silk J. 2005. See Corbelli et al. 2005, pp. 439-47 
[398] Simon R, Jackson JM, Rathborne JM, Chambers ET. 2006. Ap. J. 639:227-36

[399] Sirianni M, Nota A, Leitherer C, De Marchi G, Clampin M. 2000. Ap. J. 533:203-14

[400] Smith J, Bentley A, Castelaz M, Gehrz RD, Grasdalen GL, Hackwell JA. 1985. Ap. J. 291:571-80

[401] Smith LF, Mezger PG, Biermann P. 1978. Astron. Astrophys. 66:65-76

[402] Smith N, Bally J, Shuping RY, Morris M, Hayward TL. 2004. Ap. J. Lett. 610:L117-20

[403] Smith N, Owocki SP. 2006. Ap. J. Lett. 645:L45-48

[404] Smith N, Brooks KJ. 2007. MNRAS. In press (arXiv:0705.3053 astro-ph)

[405] Sridharan TK, Williams SJ, Fuller GA. 2005. Ap. J. Lett. 631:L73-76

[406] Stahl O, Kaufer A, Rivinius T, Szeifert T, Wolf B, et al. 1996. Astron. Astrophys. 312:539-48

[407] Stahler SW, Palla F, Ho PTP. 2000. See Mannings et al. 2000, pp. 327-51

[408] Stecklum B, Brandl B, Henning T, Pascucci I, Hayward TL, Wilson JC. 2002. Astron. Astrophys. 392:1025-29

[409] Stolte A, Brandner W, Brandl B, Zinnecker H. 2006. Astron. J. 132:253-70

[410] Stone JM, Ostriker EC, Gammie CF. 1998. Ap. J. Lett. 508:L99-102

[411] Stothers RB, Chin C-W. 1993. Ap. J. Lett. 408:L85-88

[412] Sung H, Bessell MS. 2004. Astron. J. 127:1014-28

[413] Suttner G, Yorke HW. 2001. Ap. J. 551:461-77

[414] Tamura M, Yamashita T. 1992. Ap. J. 391:710-18

[415] Tan JC. 2004. Ap. J. Lett. 607:L47-50

[416] Tan JC. 2005. See Cesaroni et al. 2005a, pp. 318-27 
[417] Tan JC, Krumholz MR, McKee CF. 2006. Ap. J. Lett. 641:L121-24

[418] Taresch G, Kudritzki RP, Hurwitz M, Bowyer S, Pauldrach AWA, et al. 1997. Astron. Astrophys. 321:531-48

[419] Terebey S, Shu FH, Cassen P. 1984. Ap. J. 286:529-51

[420] Terquem CEJMLJ. 2001. See Zinnecker \& Mathieu 2001, pp. 406-09

[421] Testi L, Palla F, Prusti T, Natta A, Maltagliati S. 1997. Astron. Astrophys. $320: 159-66$

[422] Testi L, Palla F, Natta A. 1999. Astron. Astrophys. 342:515-23

[423] Tohline JE, Durisen RH. 2001. See Zinnecker \& Mathieu 2001, pp. 40-44

[424] Trinidad MA, Curiel S, Cantó J, D’Alessio P, Rodríguez LF. 2003. Ap. J. $589: 386-96$

[425] Truelove JK, Klein RI, McKee CF, Holliman JH II, Howell LH, Greenough JA. 1997. Ap. J. Lett. 489:L179-83

[426] Turner JL, Beck SC, Crosthwaite LP, Larkin JE, McLean IS, Meier DS. 2003. Nature 423:621-23

[427] Turner NJ, Blaes OM, Socrates A, Begelman MC, Davis SW. 2005. Ap. J. $624: 267-88$

[428] Turner NJ, Quataert E, Yorke HW. 2007. Ap. J. In press (astroph/0701800)

[429] Vallée JP, MacLeod JM. 1994. Astron. J. 108:998-1001

[430] van Albada TS. 1968. Bull. Astron. Inst. Neth. 20:57-68

[431] van Altena WF, Lee JT, Lee J-F, Lu PK, Upgren AR. 1988. Astron. J. $95: 1744-54$

[432] van Bever J, Vanbeveren D. 1998. Astron. Astrophys. 334:21-28

[433] van der Tak FFS, Menten KM. 2005. Astron. Astrophys. 437:947-56 
[434] van der Tak FFS, Walmsley CM, Herpin F, Ceccarelli C. 2006. Astron. Astrophys. 447:1011-25

[435] Vanhala HAT, Boss AP, Cameron AGW, Foster PN. 1998. 29th Annu. Lunar Planet. Sci. Conf., March 16-20, 1998, Houston. Abstr. 1470

[436] Vanhala HAT, Cameron AGW. 1998. Ap. J. 508:291-307

[437] Vázquez-Semadeni E, Kim J, Shadmehri M, Ballesteros-Paredes J. 2005. Ap. J. 618:344-59

[438] Verbunt F. 1993. Annu. Rev. Astron. Astrophys. 31:93-127

[439] von Hoerner S. 1968. In Interstellar Ionized hydrogen, Proc. Symp. HII Regions, ed. Y Terzian, pp. 101-69. New York: Benjamin

[440] Walborn NR. 2003. In A Massive Star Odyssey: From Main Sequence to Supernova, ed. K van der Hucht, A Herrero, E César. IAU Symp. 212:13-21. San Francisco: ASP

[441] Walborn NR. 2007. In Massive Stars: From Pop III and GRBs to the Milky Way, ed. M Livio, E Villaver. In press (astro-ph/0701573)

[442] Walborn NR, Parker JW. 1992. Ap. J. Lett. 399:L87-89

[443] Walborn NR, Drissen L, Parker JW, Saha A, MacKenty JW, White RL. 1999. Astron. J. 118:1684-99

[444] Walborn NR, Howarth ID, Lennon DJ, Massey P, Oey MS, et al. 2002. Astron. J. 123:2754-71

[445] Walborn NR, Maíz-Apellániz J, Barbá RH. 2002. Astron. J. 124:1601-24

[446] Walborn NR, Howarth ID, Herrero A, Lennon DJ. 2003. Ap. J. 588:102538

[447] Walsh AJ, Burton MG, Hyland AR, Robinson G. 1998. MNRAS 301:64098

[448] Weidenschilling SJ, Ruzmaikina TV. 1994. Ap. J. 430:713-26 
[449] Weidner C, Kroupa P. 2004. MNRAS 348:187-91

[450] Weigelt G, Baier G. 1985. Astron. Astrophys. Lett. 150:L18-20

[451] Whitworth AP, Bhattal AS, Chapman SJ, Disney MJ, Turner JA. 1994. MNRAS 268:291-98

[452] Whitworth AP, Boffin HMJ, Francis N. 1998. MNRAS 299:554-61

[453] Williams JP, Blitz L, McKee CF. 2000. See Mannings et al. 2000, pp. $97-120$

[454] Winkler K-H, Newman M. 1980. Ap. J. 236:201-11

[455] Wolff SC, Strom SE, Dror D, Lanz L, Venn K. 2006. Astron. J. 132:749-55

[456] Wolfire MG, Cassinelli JP. 1987. Ap. J. 319:850-67

[457] Woodward PR. 1978. Annu. Rev. Astron. Astrophys. 16:555-84

[458] Wu Y, Zhang Q, Chen H, Yang C, Wei Y, Ho PTP. 2005. Astron. J. 129:330-47

[459] Wynn-Williams CG. 1982. Annu. Rev. Astron. Astrophys. 20:587-618

[460] Wyrowski F, Walmsley CM, Goss WM, Tielens AGGM. 2000. Ap. J. $543: 245-56$

[461] Yorke HW. 1986. Annu. Rev. Astron. Astrophys. 24:48-87

[462] Yorke HW. 1988. In Dust in the Universe, Proc. Conf., ed. ME Bailey, DA Williams, pp. 355-72. Cambridge, UK: Cambridge Univ. Press

[463] Yorke HW. 2002. See Crowther 2002, pp. 165-78

[464] Yorke HW, Krügel E. 1977. Astron. Astrophys. 54:183-94

[465] Yorke HW, Welz A. 1996. Astron. Astrophys. 315:555-64

[466] Yorke HW, Bodenheimer P. 1999. Ap. J. 525:330-42

[467] Yorke HW, Sonnhalter C. 2002. Ap. J. 569:846-62 
[468] Zavagno A, Deharveng L, Brand J, Massi F, Caplan J, et al. 2005. See Cesaroni et al. 2005a, pp. 346-51

[469] Zhang Q. 2005. See Cesaroni et al. 2005a, pp. 135-44

[470] Ziebarth K. 1970. Ap. J. 162:947-62

[471] Zinnecker H. 1982. NY Acad. Sci. 395:226-35

[472] Zinnecker H. 1985. In Birth and Infancy of Stars. Proc. Les Houches Summer School, ed. R Lucas, A Omont, R Stora, pp. 473-75. NATO: Knudsen.

[473] Zinnecker H. 1986. In Luminous Stars $\&$ Associations in Galaxies, ed. CWH de Loore, AJ Willis, P Laskarides, IAU Symp. 116:271-73. Dordrecht: Kluwer Academic Publishers

[474] Zinnecker H. 1991. In Fragmentation of Molecular Clouds and Star Formation, ed. E Falgarone, F Boulanger, G Duvert, IAU Symp. 147:526-32. Dordrecht: Kluwer Academic Publishers

[475] Zinnecker H. 1996. In The Interplay Between Massive Star Formation, the ISM and Galaxy Evolution. Proc. 11th IAP Astrophys. Meet., ed. D Kunth, B Guiderdoni, M Heydari-Malayeri, TX Thuan, pp. 249-58. Gif-sur-Yvette: Editions Frontieres.

[476] Zinnecker H. 2002. In The Origin of Stars and Planets: The VLT View, ed. JF Alves, MJ McCaughrean, pp. 179-86. Berlin/Heidelberg: Springer-Verlag

[477] Zinnecker H. 2003. In A Massive Star Odyssey: From Main Sequence to Supernova, ed. K van der Hucht, A Herrero, E César. IAU Symp. 212:80-90. San Francisco: ASP

[478] Zinnecker H. 2004. In The Formation and Evolution of Massive Young Star Clusters, ed. HJGLM Lamers, LJ Smith, A Nota, ASP Conf. Ser. 322:349-58

[479] Zinnecker H. 2006a. In The Scientific Requirements for Extremely Large Telescopes, ed. PA Whitelock, M Dennefeld, B Leibundgut. IAU Symp. 232:324-27. Cambridge: Cambridge University Press 
[480] Zinnecker H. 2006b. In Stellar Evolution at Low Metallicity: Mass Loss, Explosions, Cosmology, ed. HJGLM Lamers, N Langer, T Nugis, K Annuk, ASP Conf. Ser. 353:339-47

[481] Zinnecker H, McCaughrean MJ, Wilking BA. 1993. In Protostars and Planets III, ed. EH Levy, JI Lunine, pp. 429-95. Tucson: Univ. Ariz. Press

[482] Zinnecker H, McCaughrean MJ, Rayner JT. 1998. Nature 394:862-65

[483] Zinnecker H, Mathieu RD, ed. 2001. The Formation of Binary Stars, IAU Symp. 200. San Francisco: ASP

[484] Zinnecker H, Bate MR. 2002. See Crowther 2002, pp. 209-18 


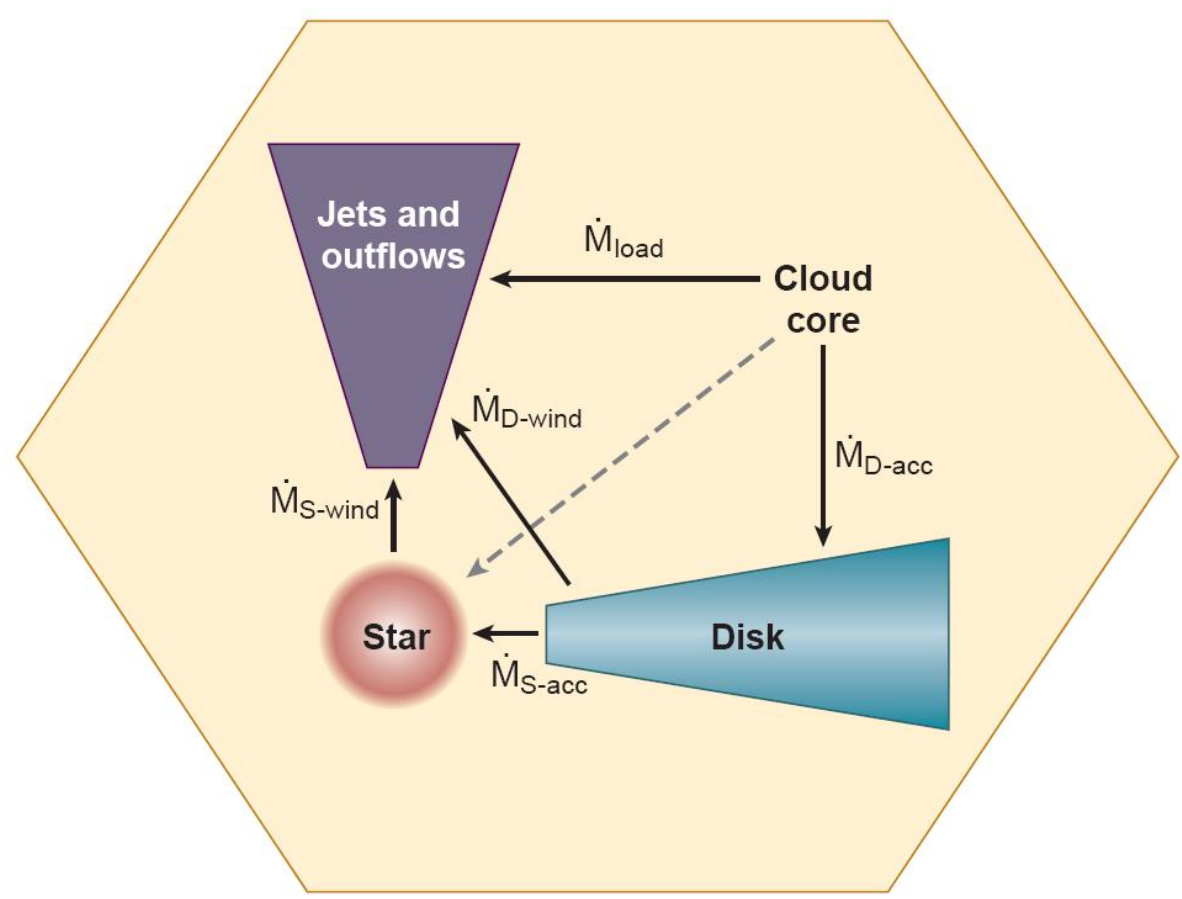

Figure 1: Accretion and mass loss as exchange between components: the accretion disk as reservoir and interface between the molecular cloud core and the forming star. 


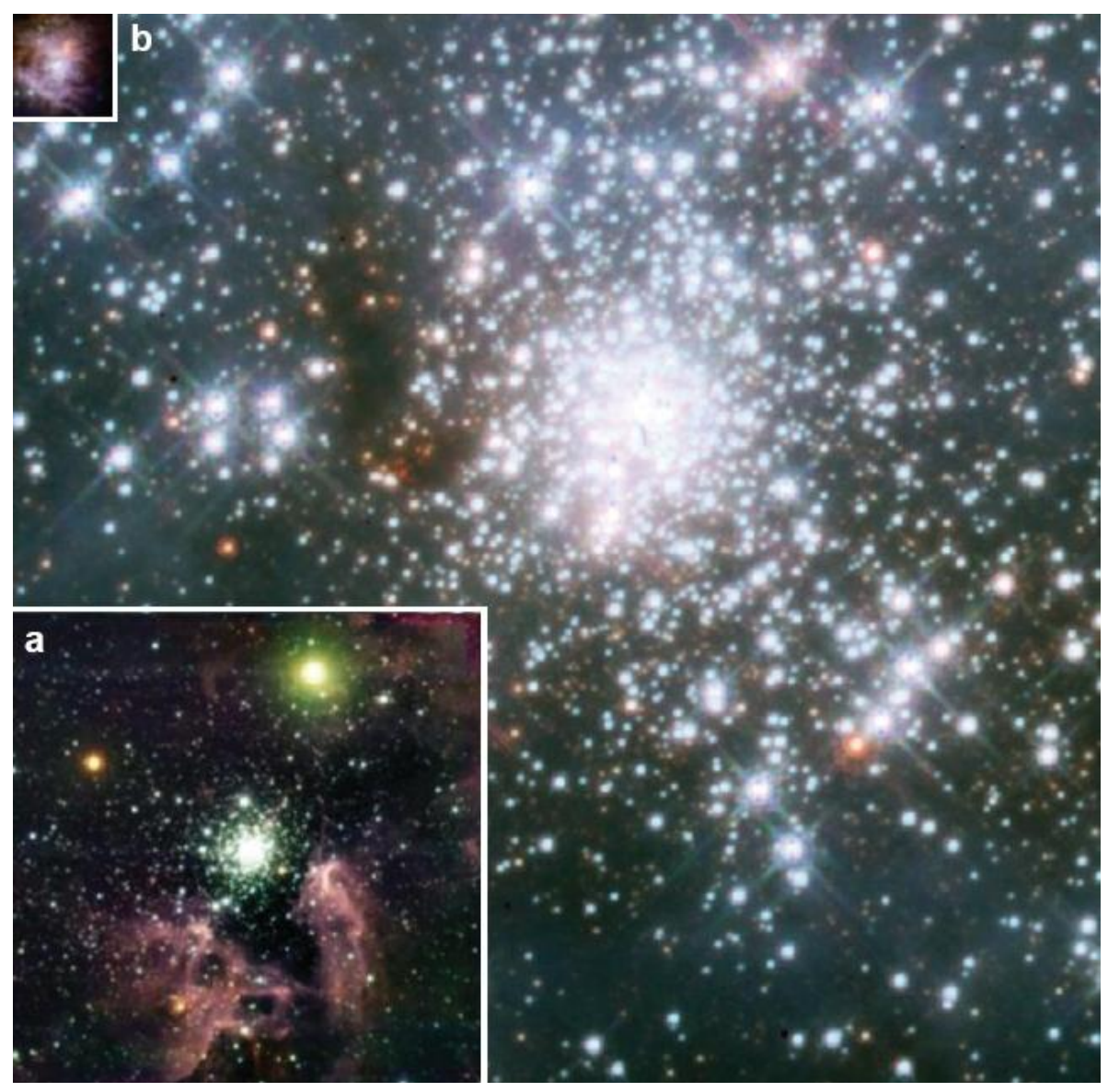

Figure 2: Hubble Space Telescope optical/IR image of the dense massive young cluster R136/30 Dor (courtesy of M.J. McCaughrean; FOV $\sim 30 \operatorname{arcsec} \times 30 \operatorname{arcsec}$ or $7.5 \mathrm{pc} \times 7.5 \mathrm{pc}$ ). Dozens of massive $\mathrm{O}$ stars are found crowded within the half-light radius of $2 \mathrm{pc}$ (Brandl et al. 1996). (a) A VLT image of NGC 3603 (Brandl et al. 1999) and (b) a VLT image of the Trapezium Cluster in Orion (McCaughrean 2001) are shown, as these two galactic clusters would be seen if they were located at the distance of R136 in the Large Magellanic Cloud $(50 \mathrm{kpc})$ and imaged with similar angular resolution (see Zinnecker 2002). 


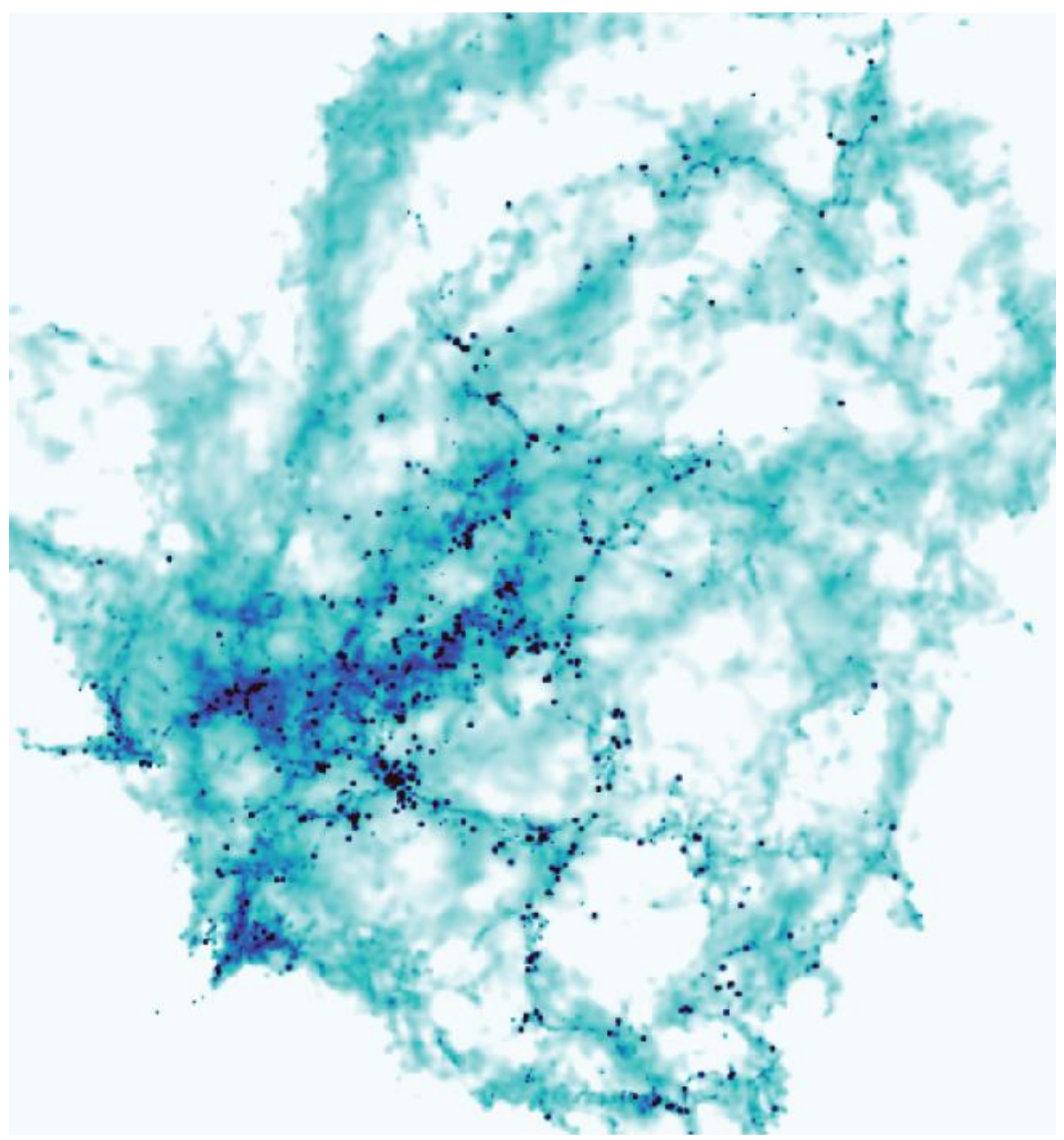

Figure 3: Collapse and fragmentation of a giant molecular cloud, simulated with smoothed particle hydrodynamics (SPH) using sink particles (I.A. Bonnell, P.C. Clark \& H. Zinnecker, in preparation). For a description of SPH, see the box. Plotted is the spatial distribution of gas column density, color-coded such that deep blue refers to the highest values. The initial conditions for this three-dimensional simulation included a molecular gas cloud of mass $10^{6} \mathrm{M}_{\odot}$ and diameter 100 pc, somewhat centrally condensed (factor of 20, with a Gaussian radial profile). The cloud's turbulent kinetic energy was equal to its gravitational energy. Note the filamentary structure and the associated dense cores (blue dots). These cores technically are represented by sink particles $(\sim 2400$ at this stage of evolution). With typical masses of 10 to $100 \mathrm{M}_{\odot}$ and typical sizes of $0.1 \mathrm{pc}$ they could be the initial fragments for high-mass star formation. A total of 2.5 million SPH particles was used. Some similarity to cosmological simulations of structure formation is noted. 


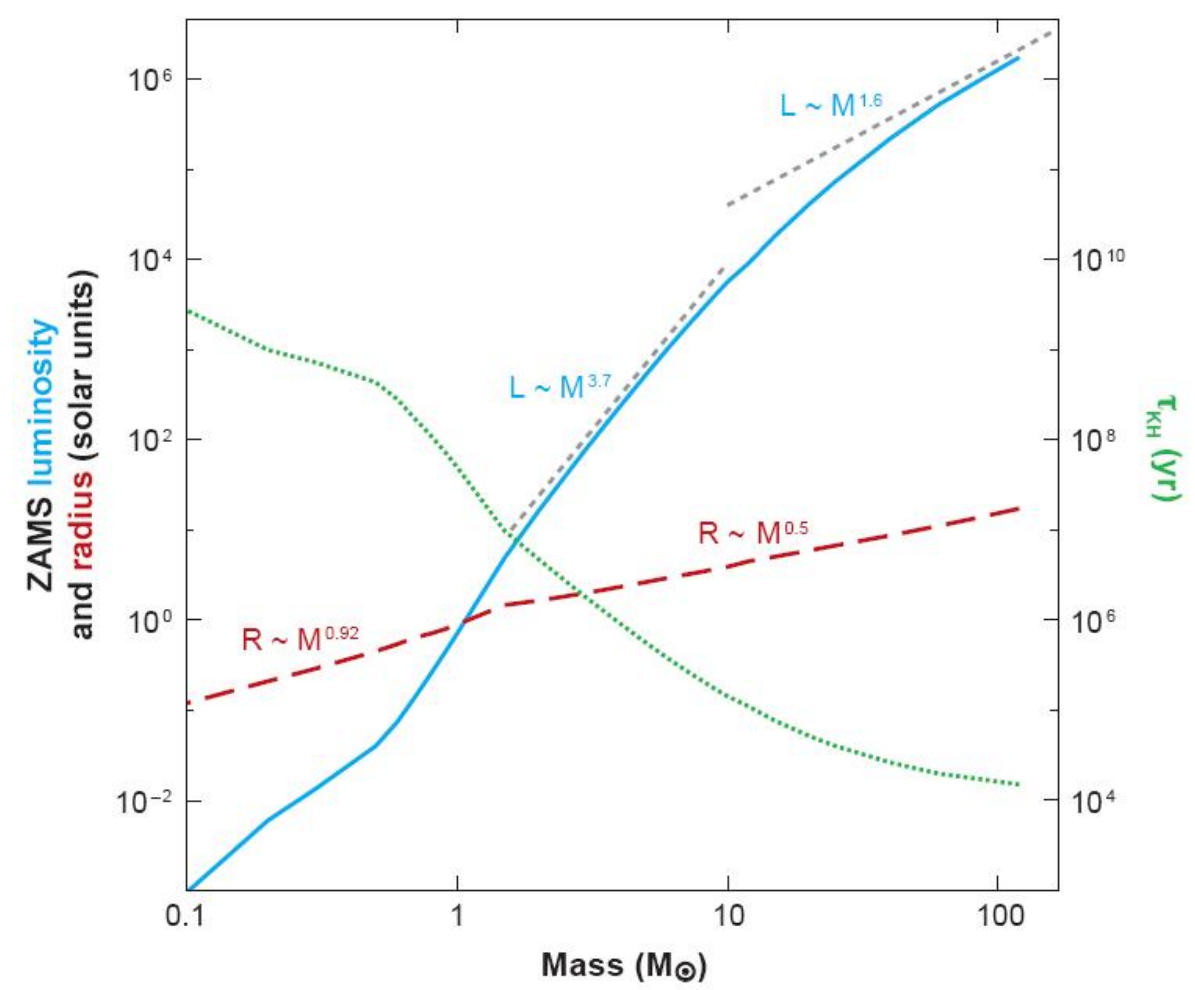

Figure 4: ZAMS luminosity (blue), ZAMS radius (red dashed), and KelvinHelmholtz quasi-static gravitational contraction timescale toward the ZAMS (green dotted) as a function of stellar mass (ZAMS means zero-age main sequence). These values are extracted from $\mathrm{t}=0 \mathrm{yr}$ models given by Meynet \& Maeder (2005) for rotating stars $\mathrm{M}_{*} / \mathrm{M}_{\odot} \geq 12$ with mass loss, by Pietrinferni et al. (2004) for stars $0.5 \leq \mathrm{M}_{*} / \mathrm{M}_{\odot} \leq 10$, and for completeness from unpublished tracks by Yorke for masses $\mathrm{M}_{*}=0.1,0.2$, and $0.3 \mathrm{M}_{\odot}$, using the computer code provided in the book by Bodenheimer et al. (2007). ZAMS radii are well represented by two power laws with a break at $\mathrm{M}_{*} / \mathrm{M}_{\odot}=1.5$. The power law slope of the luminosity-mass relation for massive stars varies from about 3.7 to 1.6 in the range $8<\mathrm{M}_{*} / \mathrm{M}_{\odot}<120$. 


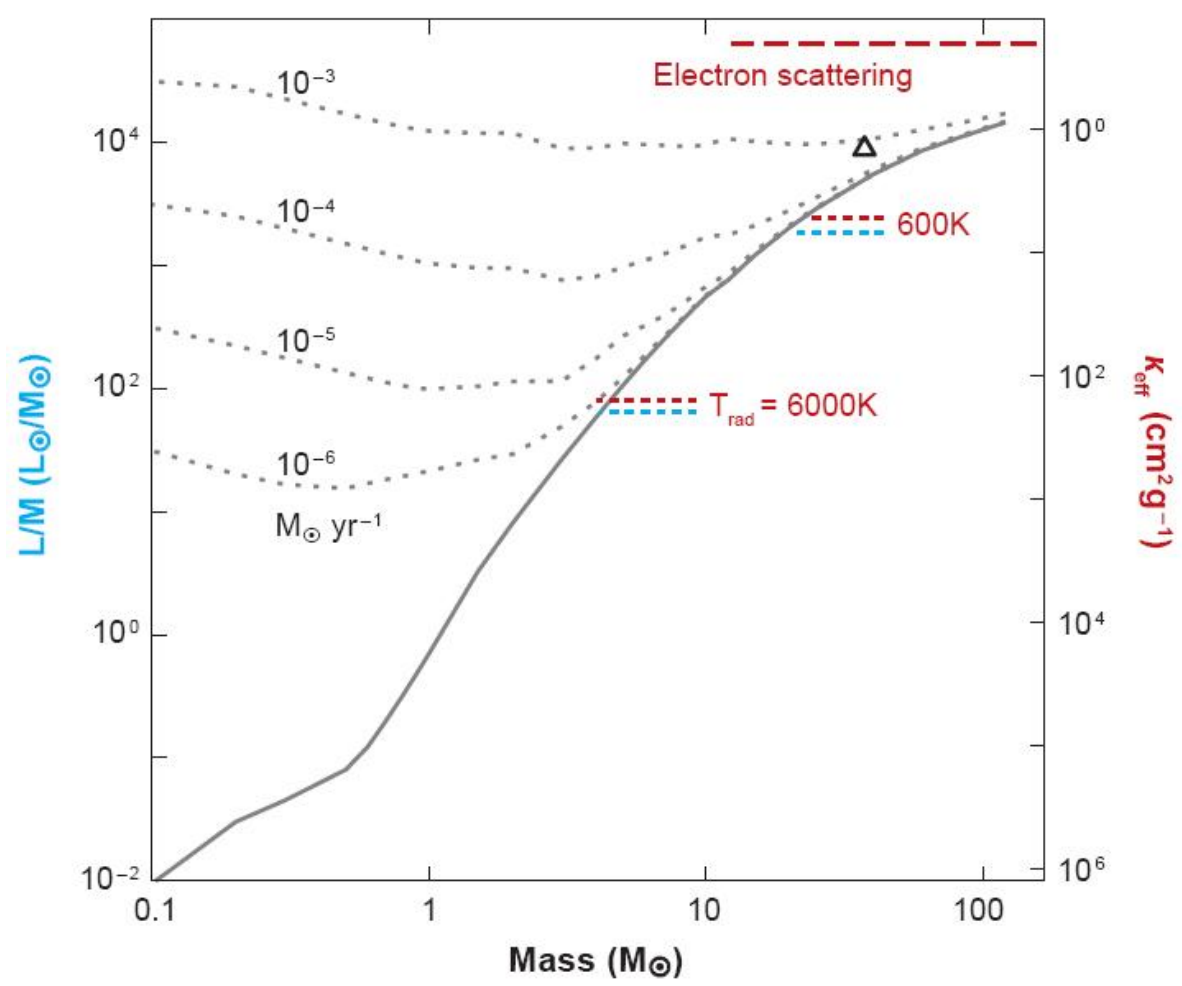

Figure 5: ZAMS luminosity to mass ratio (left scale) and corresponding critical effective opacity (right scale) as defined by Equation $\mathbf{3}$ as a function of stellar mass. The solid grey line depicts the ZAMS models shown in Figure 4. Dotted grey lines indicate the total luminosity (including accretion luminosity) of stars accreting at the indicated constant rate as discussed in the text (cf. Figure 8). The triangle denotes the position of an O5V star (see Figure 9). Dashed lines denote the opacities of dusty gas in the light of black body sources at the temperature indicated (for two different grain types, see Figure 6) and the contribution from electron scattering in a fully ionized plasma. Because of the combined effect of UV lines (UV line blanketing), the actual opacity of a hot plasma can be greater than that from electron scattering alone. This may be relevant for the existence of an upper stellar mass limit. 


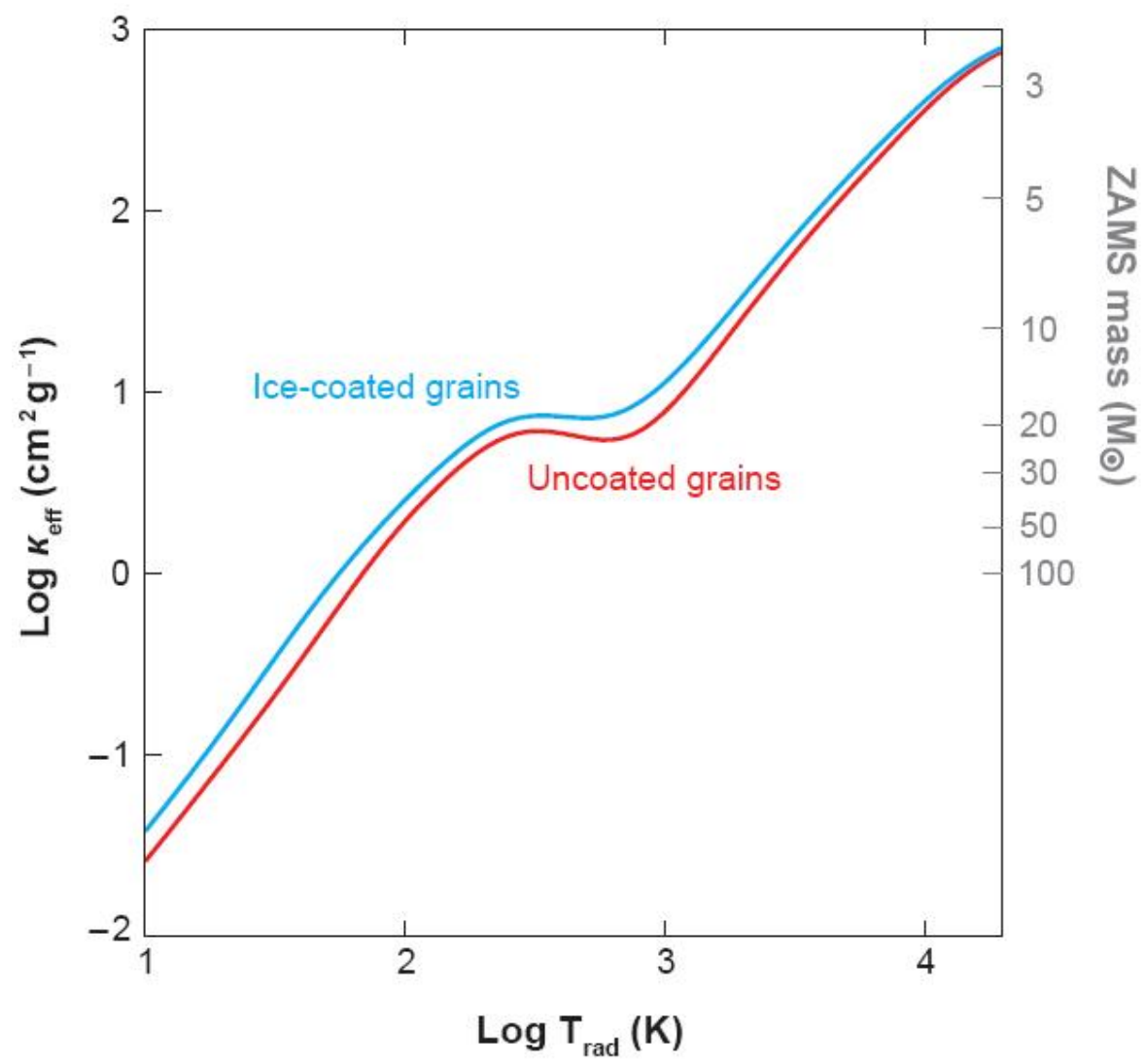

Figure 6: Planck-weighted mean effective opacity of dusty gas, using the (Preibisch et al. 1993) dust model with ice-coated grains (blue curve) and grains without ice mantels (red curve), assuming black-body radiation at the temperature $\mathrm{T}_{\mathrm{rad}}$ and solar abundances. The right-hand scale (grey) is based on the ZAMS models shown in Figure 5 (solid grey line). 


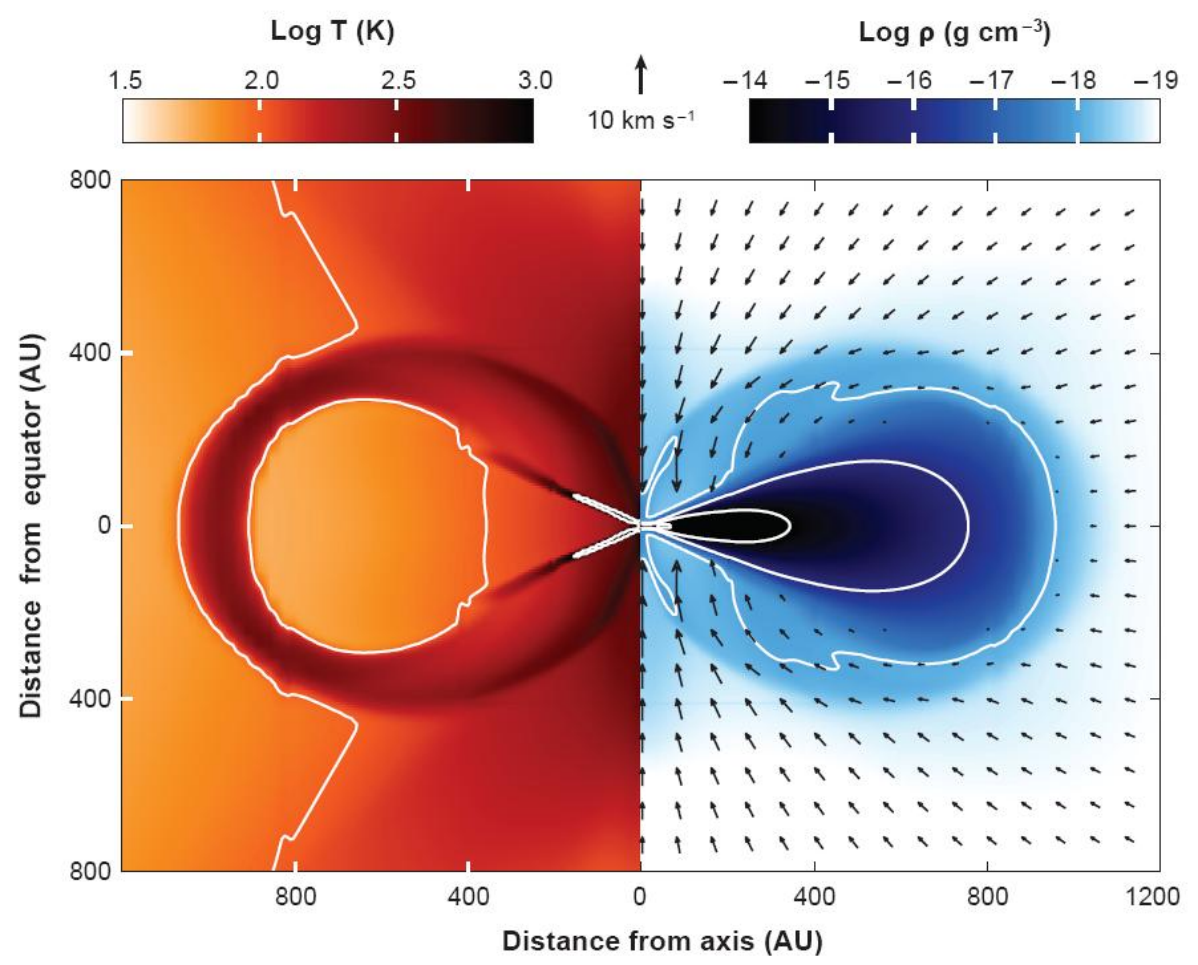

Figure 7: Edge-on cut through an accreting protostar and its circumstellar disk and envelope. The protostar is located at $(0,0)$ and is too tiny to see. Temperature (red scale, upper-left), density (blue scale, upper-right), and velocity (length and direction of arrows) distributions of the accreting material are displayed (case F of Yorke \& Bodenheimer 1999, recalculated using an improved version of their code). White contour lines are plotted for $\log \mathrm{T}=2.0,2.5$, and 3.0 and for $\log \rho=-18,-16,-14$, and -12 . At this evolutionary time $\mathrm{t}=65,000 \mathrm{yr}$ after formation of the protostellar core, $7.0 \mathrm{M}_{\odot}$ of material have accreted onto the protostar, $2.8 \mathrm{M}_{\odot}$ are in the disk, and $0.2 \mathrm{M}_{\odot}$ are in the infalling envelope. 


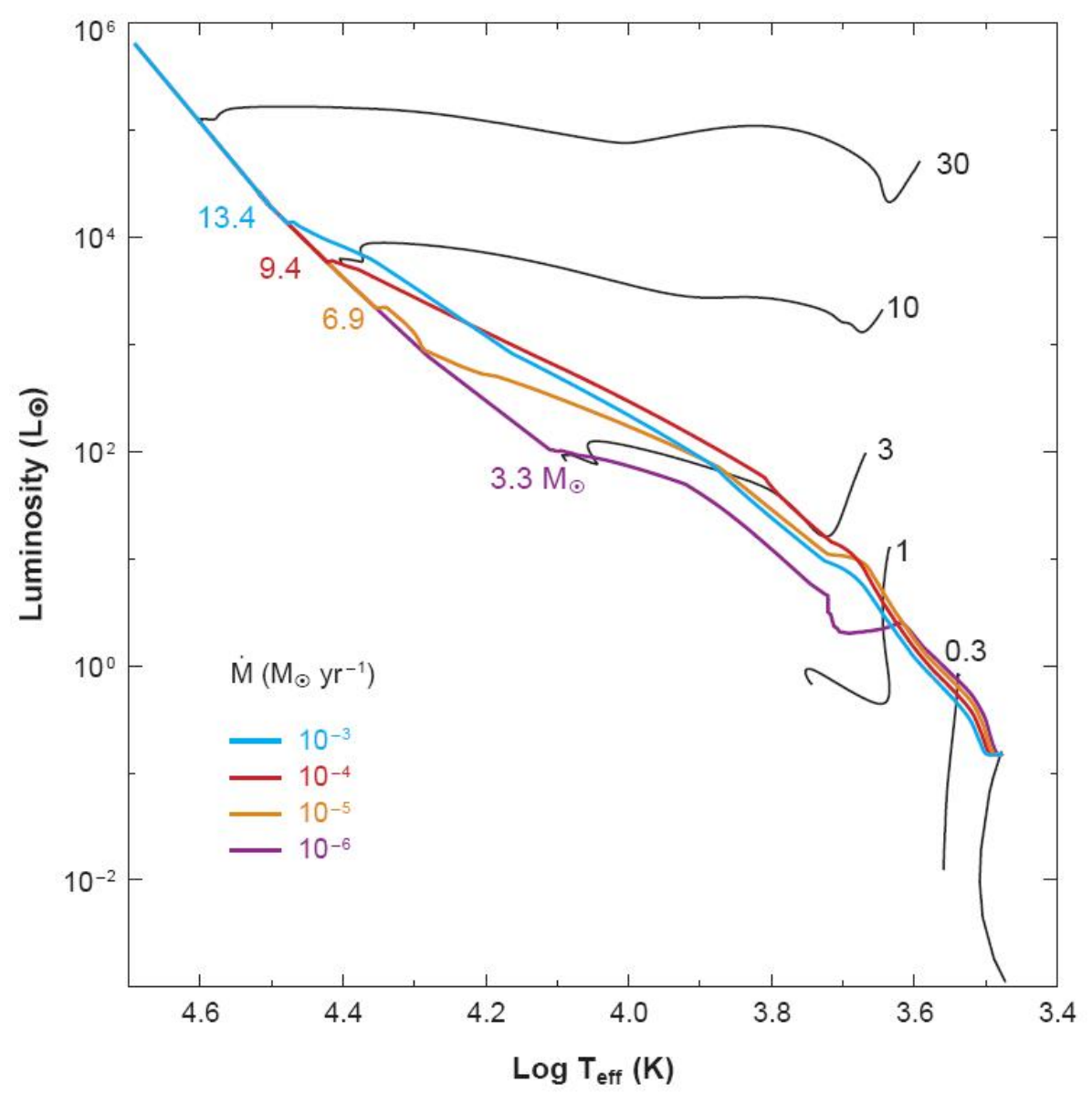

Figure 8: Evolutionary tracks in the HR diagram for (proto-)stars accreting at a constant rate (colored lines) are contrasted to the tracks of non-accreting stars (black lines). All accreting tracks are assumed to begin at the birthline of an equilibrium deuterium burning $0.1 \mathrm{M}_{\odot}$ pre-main-sequence star. Non-accreting tracks up to $\mathrm{H}$ burning were calculated by Yorke for $\mathrm{M}_{*} / \mathrm{M}_{\odot}=0.1,0.3,1,3,10$, and 30, using the computer code supplied with the book by Bodenheimer et al. (2007). [Adapted from Yorke (2002)] 


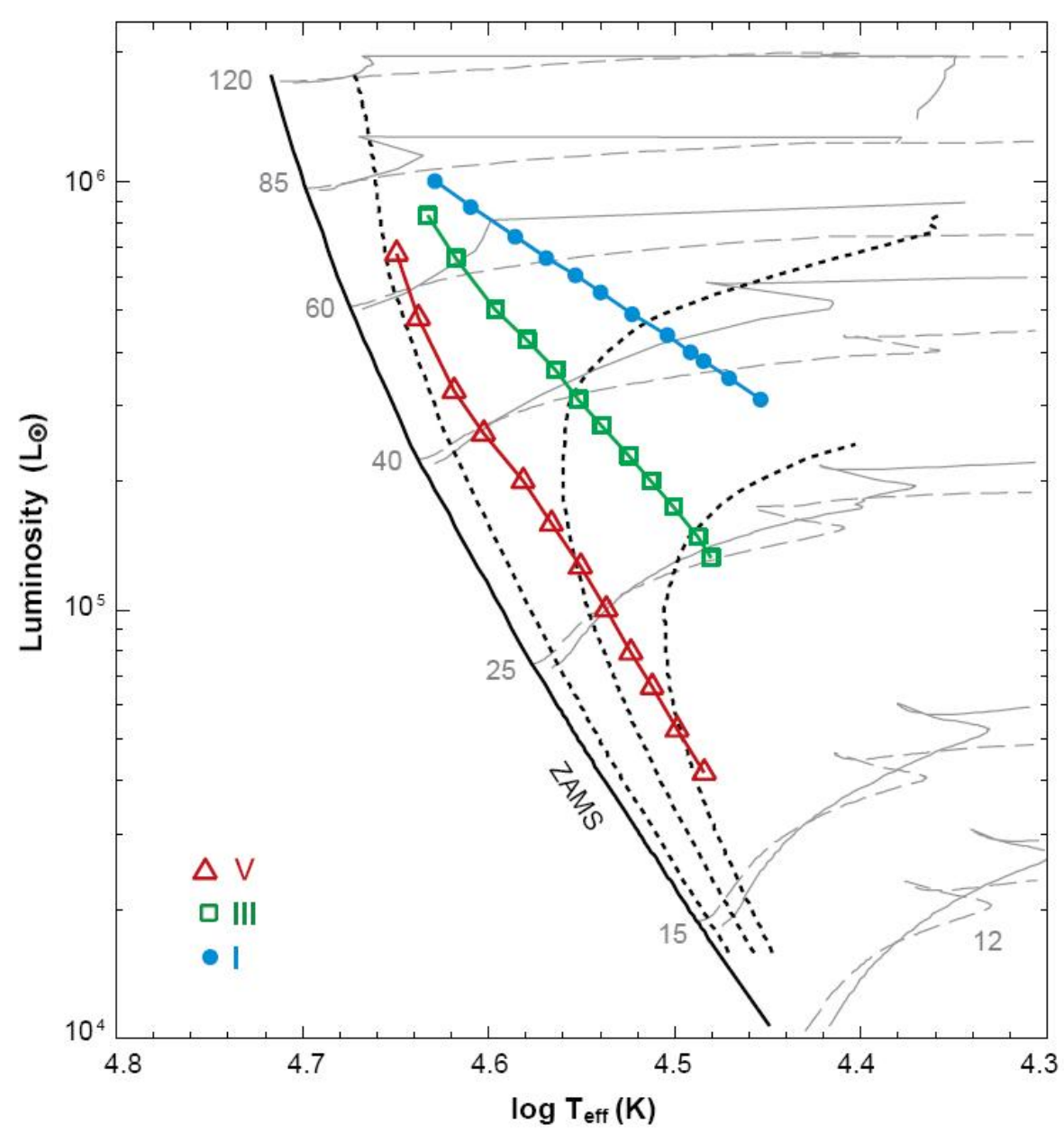

Figure 9: Luminosity and effective temperature calibration of luminosity classes I (blue-filled circles), III (green squares), and V (red triangles) for stars of spectral classes O3, O4, O5, O5.5, O6, O6.5, O7, O7.5, O8, O8.5, O9, and O9.5. For comparison, theoretical evolutionary tracks from Meynet \& Maeder (2003) for non-rotating (dashed grey) and for rotating stars (continuous grey) with mass loss are labeled by their starting ZAMS mass. Meynet et al. (1994) isochrones for non-rotating stars are also plotted for 0 (solid black line labeled ZAMS), 1, 3 , and $5 \mathrm{Myr}$ (dotted black lines). [Adapted from Figure 14 of Martins, Schaerer \& Hillier (2005)] 


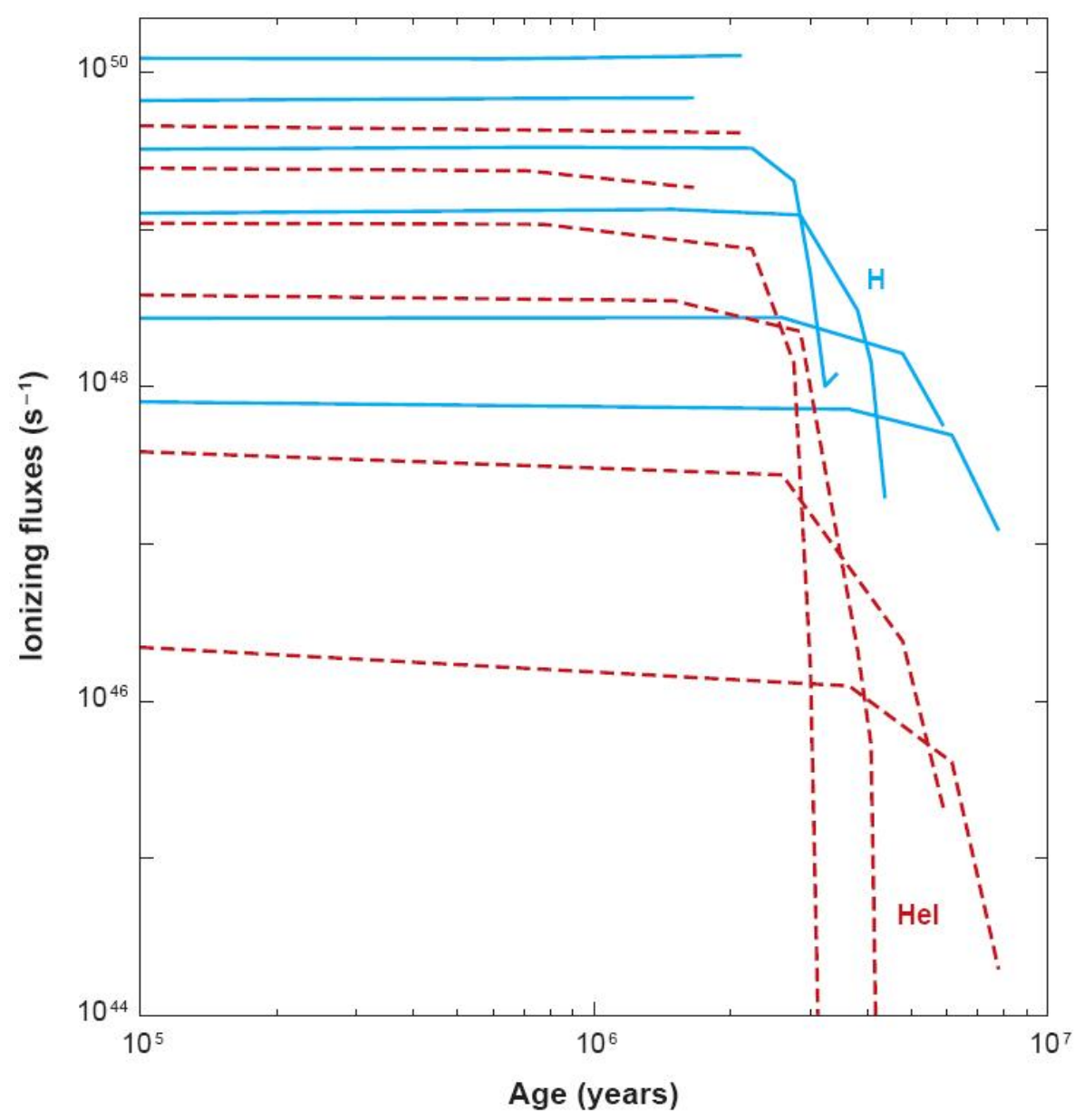

Figure 10: Flux of radiation that can ionize hydrogen (blue lines) and neutral helium (red dashed lines) as a function of age (counted from the moment of arrival on the ZAMS), for stars of ZAMS masses (top to bottom) of $\mathrm{M}_{*} / \mathrm{M}_{\odot}=120$, 85, 60, 40, 25, and 20. Mass loss is included in the evolution. [Based on models from Schaerer \& de Koter (1997)] 


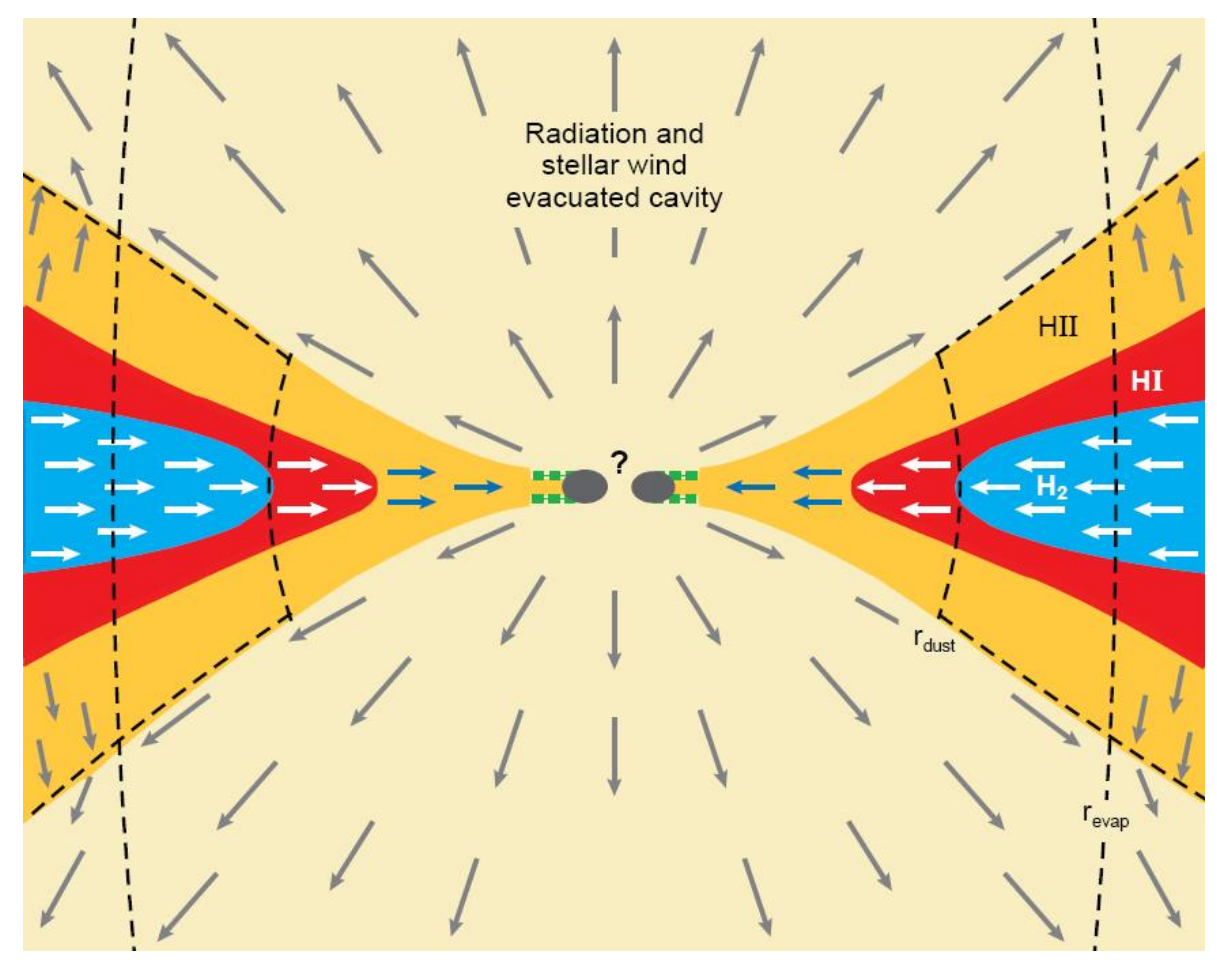

Figure 11: The inner accretion disk around a close massive accreting binary pair: inward radial flow is allowed in the equatorial plane. A polar cavity is evacuated by a combination of radiation and the stellar wind. The disk is self-shielded from the intense EUV field by an ionization front separating HII and $\mathrm{HI}$ gas; and from the FUV field interior to the $\mathrm{HI} / \mathrm{H}_{2}$ interface by dust, molecular hydrogen, and CO. The dust is destroyed at $r_{\text {dust }}$. Interior to $r_{\text {evap }}$, the radius where the sound speed exceeds the escape velocity, even the ionized gas is gravitationally bound. Sizes are not to scale. 


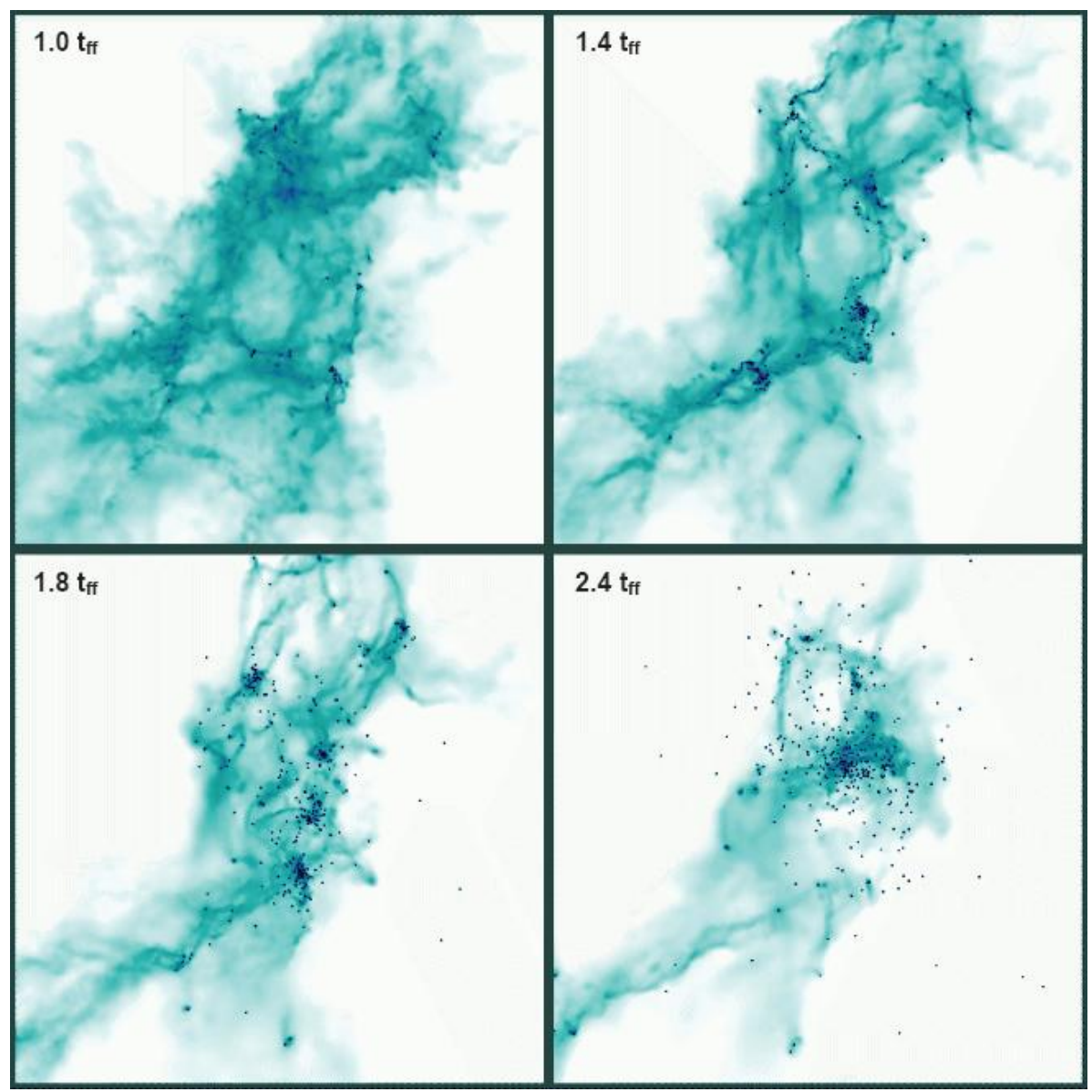

Figure 12: Time-dependent evolution of a turbulent, self-gravitating $1000-\mathrm{M}_{\odot}$ cloud showing the formation of sheets, filaments, and cores, the latter of which become gravitationally unstable. Newly formed stars are shown in dark blue, the gas is shown in blue-green. Stars tend to cluster, they continue to accrete material in competition with other stars. Times shown are 1.0, 1.4, 1.8, and 2.4 initial free-fall times $\left(t_{\mathrm{ff}}\right)$, from left to right and top to bottom. [Adapted from Bonnell, Bate \& Vine (2003)]. 


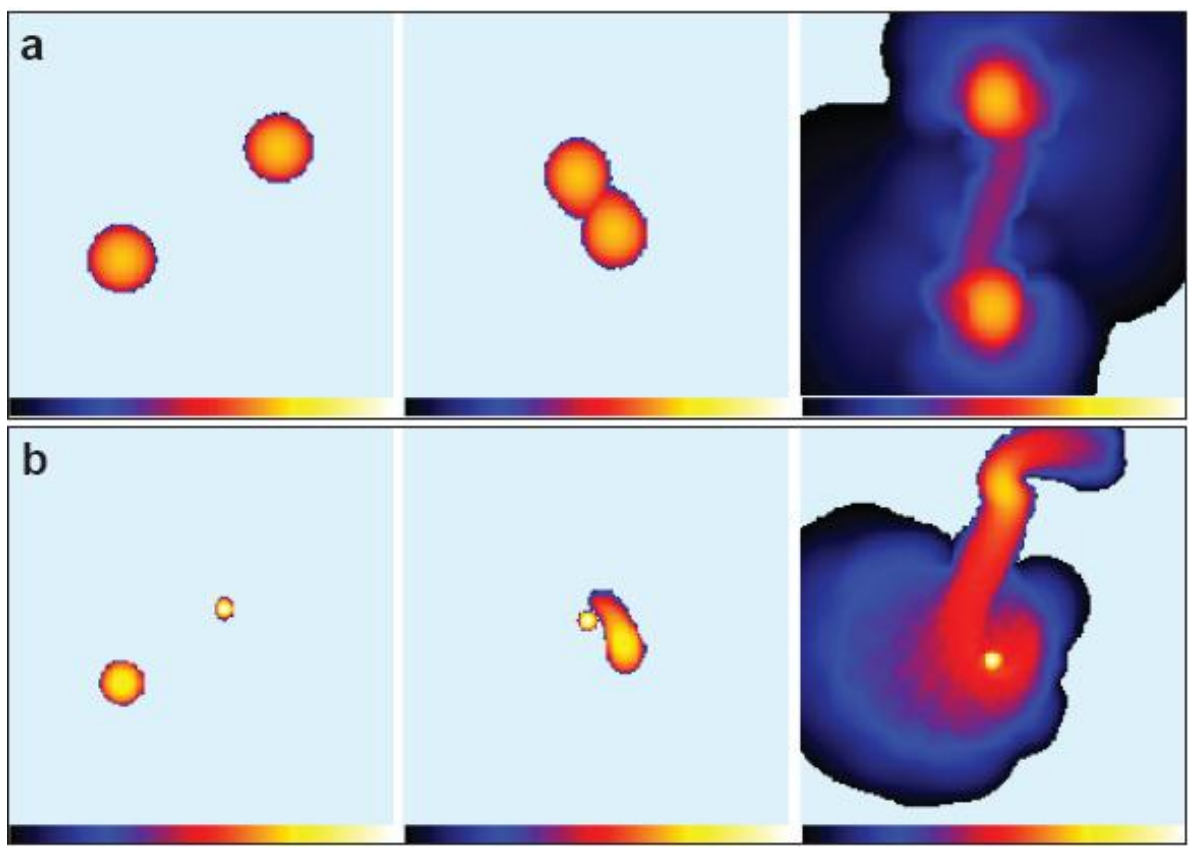

Figure 13: (a) A grazing encounter between two $3 \mathrm{M}_{\odot}$ premain-sequence stars that results in the formation of a binary $\left(\mathrm{r}_{\min }=25.8\right.$ solar radii). (b) A detached encounter between a $3 \mathrm{M}_{\odot}$ premain-sequence star and a $10 \mathrm{M}_{\odot}$ zero age main-sequence star with the same minimum periastron distance as in the (a) encounter. The stars have radii of 12.9 and 3.92 solar radii, respectively. The greater density of the $10 \mathrm{M}_{\odot}$ star results in tidal disruption of the low-density $3 \mathrm{M}_{\odot}$ star to form a disk around the massive star. The encounters have zero relative velocity at infinity (i.e., they are parabolic encounters). [ Adapted from Zinnecker \& Bate (2002), Davies et al. (2006)]. The cross section for a subsequent collision with (say) another $10 \mathrm{M}_{\odot}$ star is significantly increased, and a runaway collisional growth in mass is possible. This is the so-called 'shred and add' process. 


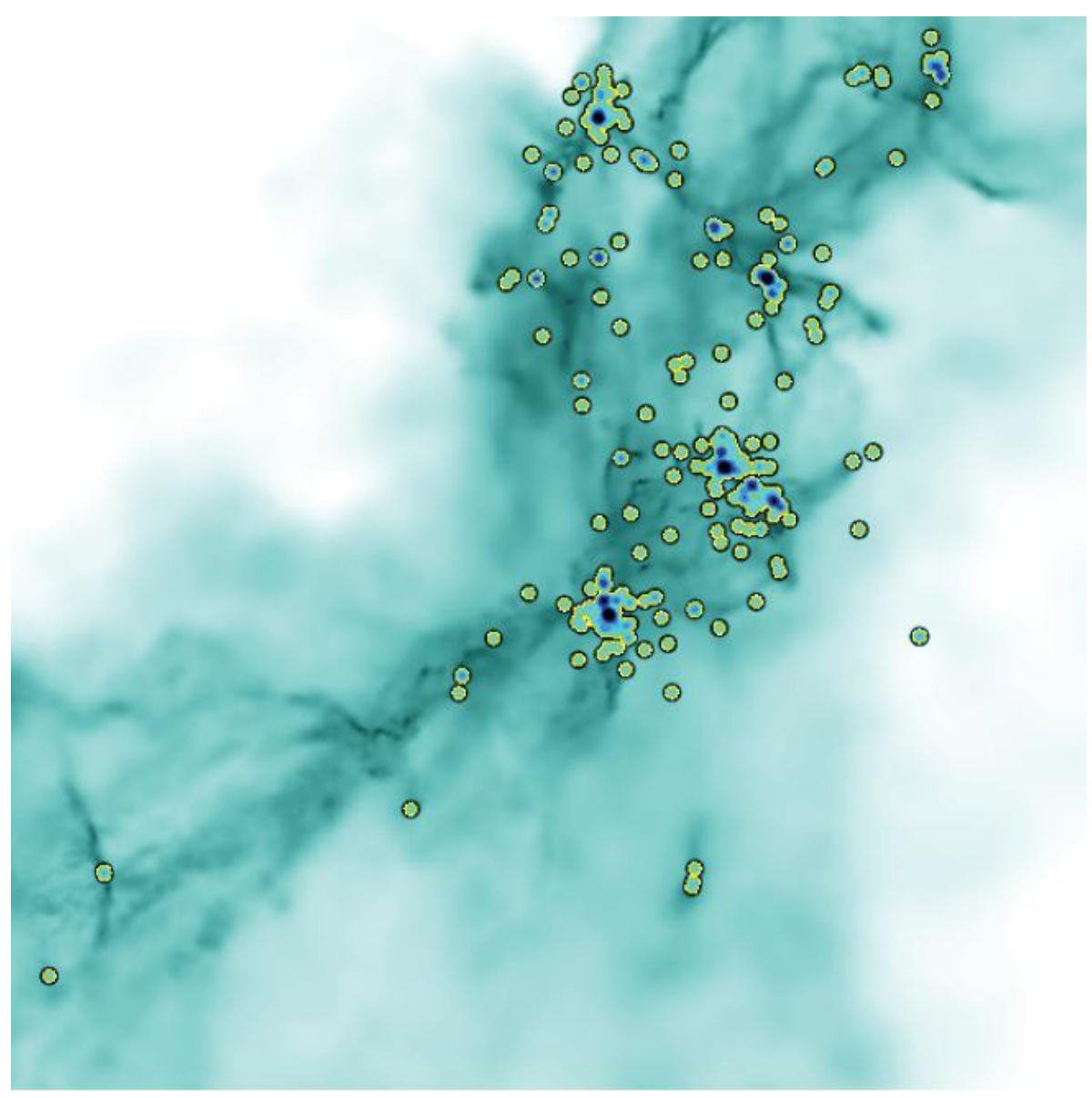

Figure 14: Massive stars (dark blue circles) are formed in the center of individual subclusters of low-mass stars (light circles) because of competitive accretion. These subclusters evolve by merging with the other subclusters. The final state of the simulation is a single, centrally condensed cluster with little substructure but with 4 massive stars, one from each subcluster. This then is a model for the origin of Trapezium-type systems. [Adapted from Bonnell, Vine \& Bate (2004)] 


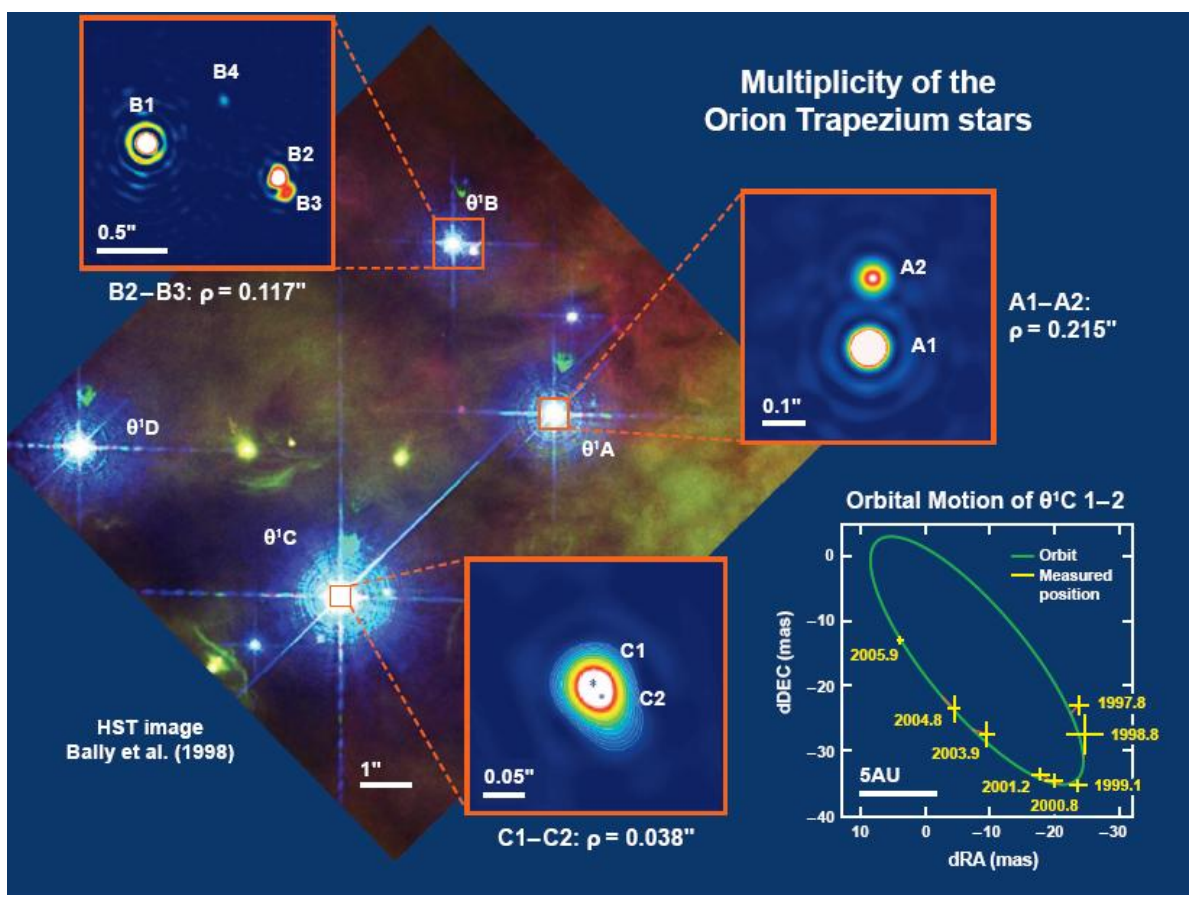

Figure 15: The multiple star systems of the Orion Trapezium as revealed by bispectrum speckle interferometry. [Courtesy of G. Weigelt \& Th. Preibisch; inserts adapted from Schertl et al. (2003) and Kraus et al. (2007)] 


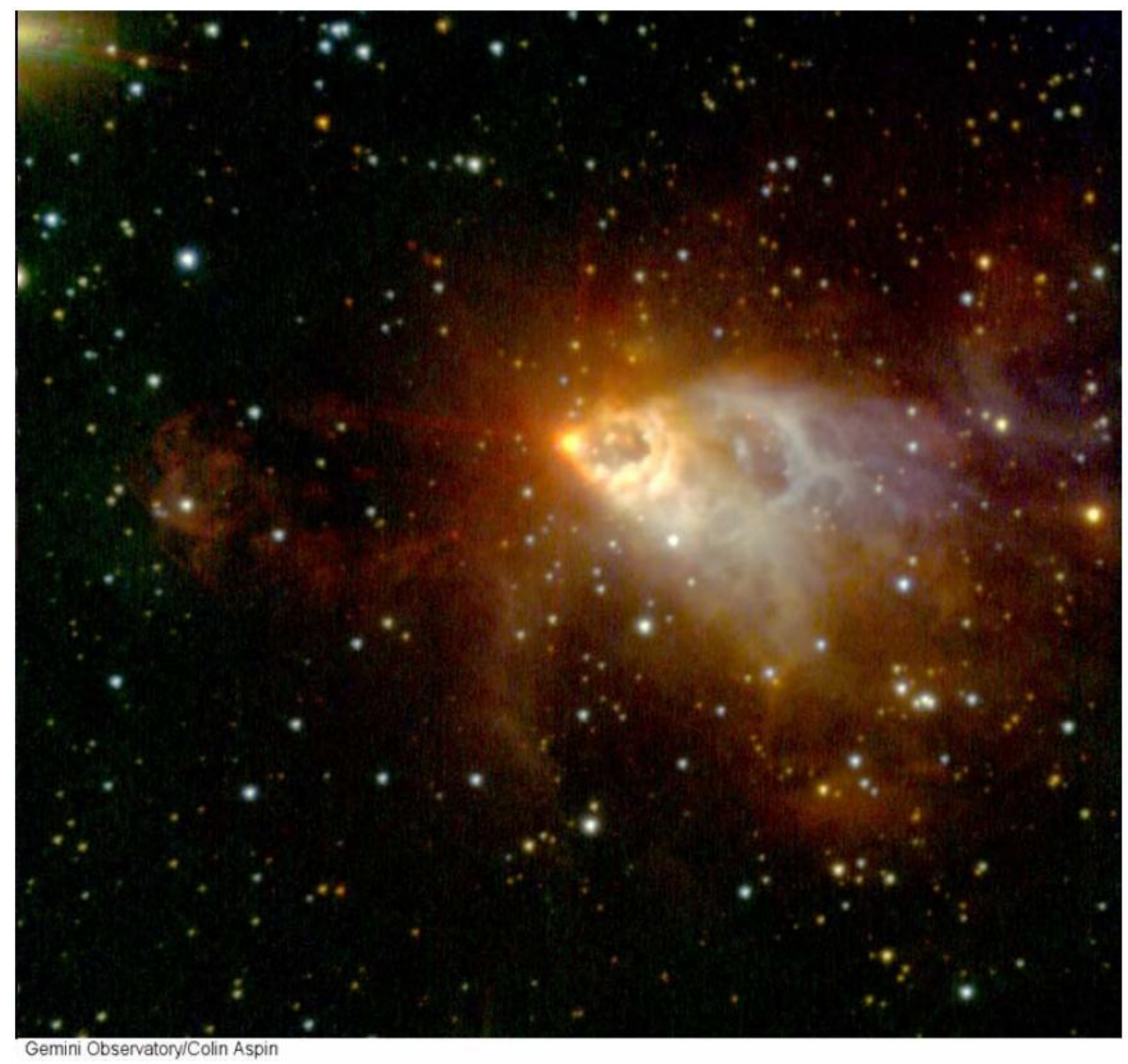

Figure 16: A wide-field JHKs composite image of the AFGL 2591 massive outflow taken with the NIRI camera at the Gemini North telescope in excellent seeing (courtesy of C. Aspin and Gemini Observatory; FOV $\sim 2$ arcmin, seeing 0.35 arcsec). The broad-band Ks filter includes the ro-vibrational molecular hydrogen $\mathrm{v}=1-0 \mathrm{~S}(1)$ emission line at $2.12 \mu \mathrm{m}$, which is indicative of shockexcited gas. However, much of the nebulosity is likely seen in reflection because it is also detected in $\mathrm{J}$ and $\mathrm{H}$. Note the multiple poorly-collimated flows, loops, and cavities emanating to the west from this massive (ca. $20 \mathrm{M}_{\odot}$ ) star (see also the speckle image in Preibisch et al. 2003 from the $6 \mathrm{~m}$ SAO telescope). Nothing of this kind is seen to the east of the source, except for a few faint bow-shocks, as the (redshifted) counterflow is deeply embedded and probably partly obscured by a dense circumstellar disk (Trinidad et al. 2003) around this very young star. The fact that the flow and counterflow both reach the same distance of $\sim 40 \operatorname{arcsec}(0.2 \mathrm{pc})$ from the central source indicates that this is intrinsically a bipolar outflow, confirmed by high-velocity CO radio observations (Lada et al. 1984; see also the $\mathrm{H}_{2}$ and optical spectroscopic observations of the associated Herbig-Haro objects by Tamura \& Yamashita 1992 and Poetzel, Mundt \& Ray 1992, respectively). It seems that the bipolar outflow from this late O-type massive star has both a well-collimated and a wide-angle component! 


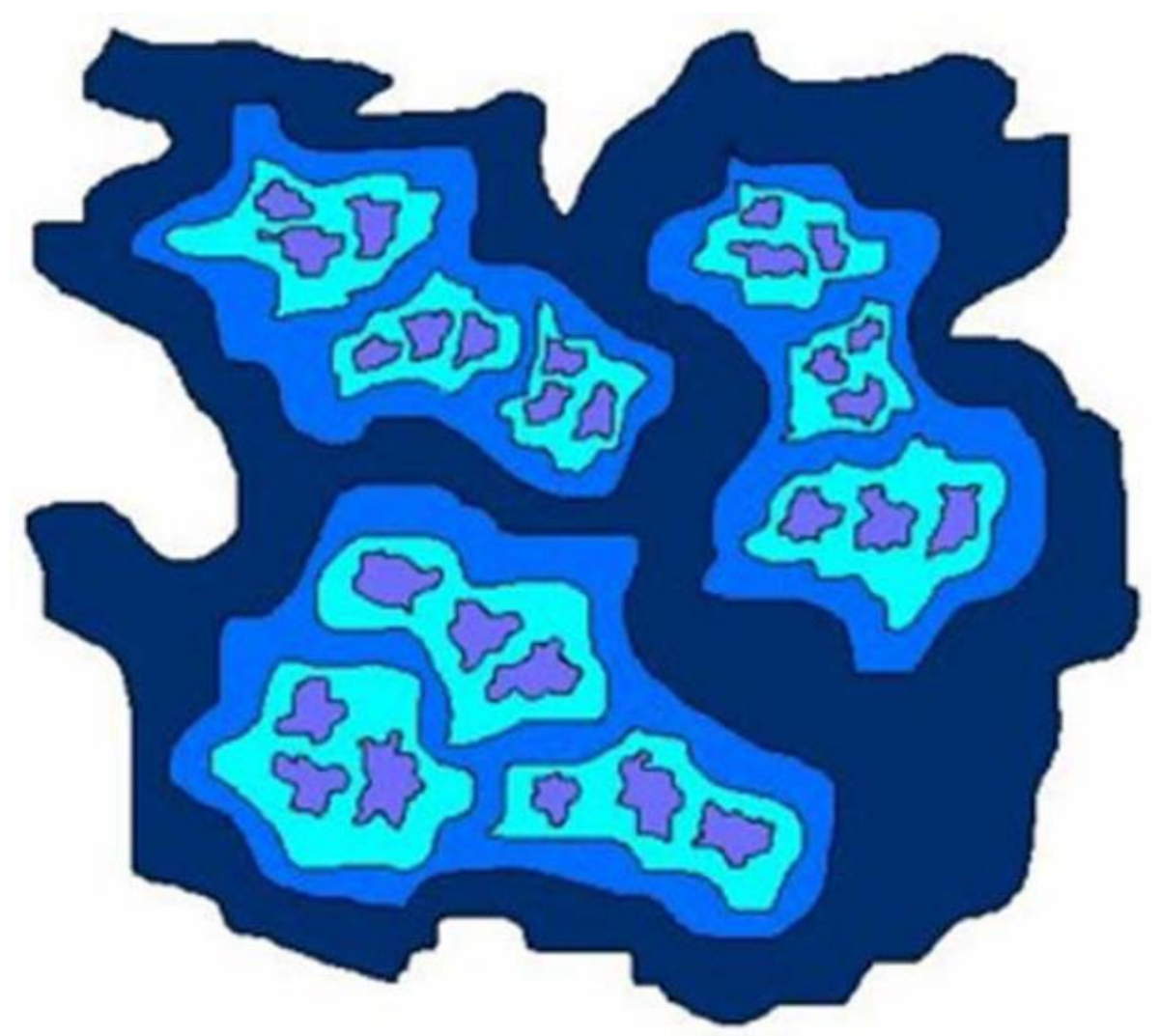

Figure 17: Schematic representation of a self-similar fractal hierarchy of dense gas cores in a turbulent molecular cloud with three branches per level (courtesy J. Melnick). The chance of randomly picking a core of mass $\mathrm{M}$ in a cloud with such hierarchical structure is proportional to $1 / \mathrm{M}$. Because the cloud branches correspond to log intervals, random sampling yields a mass spectrum of (prestellar) cores with equal mass in equal logarithmic intervals, i.e., a power-law $\mathrm{dN} / \mathrm{d} \log \mathrm{M}$ with index -1 . If there is competition for mass, i.e., if some smaller (and presumably denser) cores are turned into stars before the bigger cores, of which they are part, have time to collapse, then there will no longer be enough mass available to form a star with the mass of the undiminished larger core. Thus, high-mass cores (stars) get depleted from the mass function if low-mass cores (stars) form first. The faster formation of low-mass stars, combined with the mass depletion of subparts inside clouds, converts the power-law mass spectrum with a power index of -1 into one with an index of -1.35 (or so), i.e., into a Salpeter initial mass function. Note that in this reasoning (following Elmegreen 1997 ) it was assumed that a fixed fraction of each and every core mass is turned into stellar mass (single, binary, or multiple). 


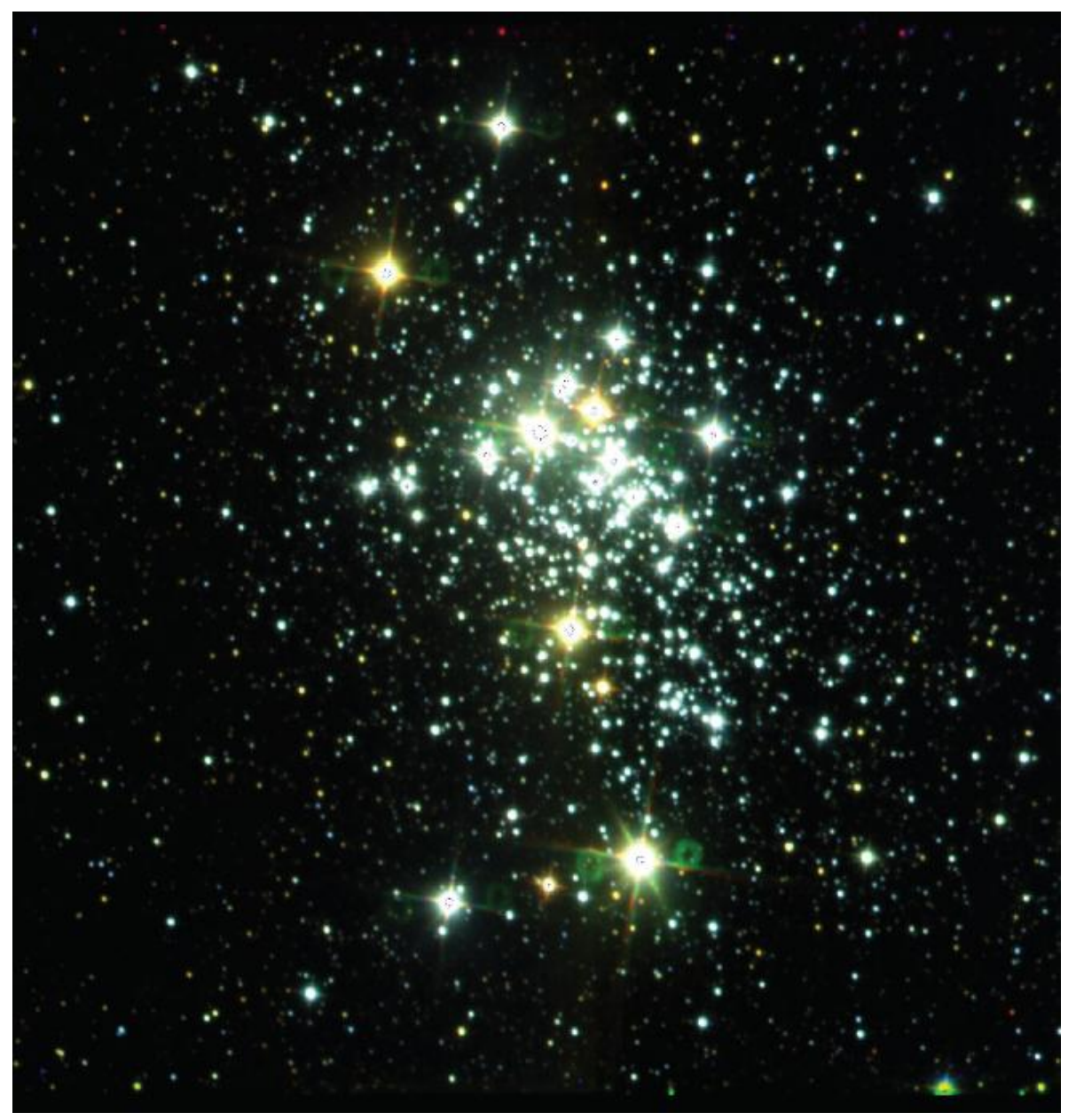

Figure 18: Composite colour JHKs image of the $\sim 4$ Myr old Galactic starburst cluster Westerlund 1 (Wd 1) in Cyg OB2 obtained with the ESO NTT telescope (courtesy W. Brandner). This obscured object is held to be the most massive young cluster in the Galaxy, with ca. 100 O-type stars and a mass of at least $20,000 \mathrm{M}_{\odot}$ (see Knödelseder 2000, Clark et al. 2005, Mengel \& Tacconi-Garman 2007, Brandner et al. 2007). A number of massive eclipsing binary systems have been identified (Bonanos 2007) as well as a very massive runaway star, an Oftype supergiant, whose origin can be traced back to the cluster (Comerón \& Pasquali 2007). Like the Orion Nebula Cluster, or the NGC 3603 and 30 Dor central clusters, Wd 1 is strongly mass segregated with the high mass stars being more centrally concentrated than the low mass stars. The field of view is $4^{\prime} \times 4^{\prime}$, corresponding to $4.2 \mathrm{pc} \times 4.2 \mathrm{pc}$ at the distance of $\mathrm{Wd} 1$. 